\title{
CMOS Implementation Of Active And Passive LC-VCO
}

\author{
by
}

Albert Chiu Yiu Chun, B.Eng.

SUBMITTED IN PARTIAL FULFILLMENT OF THE

REQUIREMENTS FOR THE DEGREE OF

MASTER OF APPLIED SCIENCE

AT

CARLETON UNIVERSITY

DEPARTMENT OF ELECTRONICS

OTTAWA, ONTARIO

MAY 2008

Copyright by Albert Chiu Yiu Chun, 2008 


$\begin{array}{ll}\begin{array}{l}\text { Library and } \\ \text { Archives Canada }\end{array} & \begin{array}{l}\text { Bibliothèque et } \\ \text { Archives Canada }\end{array} \\ \begin{array}{l}\text { Published Heritage } \\ \text { Branch }\end{array} & \begin{array}{l}\text { Direction du } \\ \text { Patrimoine de l'édition }\end{array} \\ \begin{array}{l}\text { 395 Wellington Street } \\ \text { Ottawa ON K1A 0N4 } \\ \text { Canada }\end{array} & \begin{array}{l}\text { 395, rue Wellington } \\ \text { Ottawa ON K1A 0N4 } \\ \text { Canada }\end{array}\end{array}$

Your file Votre référence ISBN: 978-0-494-40635-9 Our file Notre référence ISBN: 978-0-494-40635-9

NOTICE:

The author has granted a nonexclusive license allowing Library and Archives Canada to reproduce, publish, archive, preserve, conserve, communicate to the public by telecommunication or on the Internet, loan, distribute and sell theses worldwide, for commercial or noncommercial purposes, in microform, paper, electronic and/or any other formats.

The author retains copyright ownership and moral rights in this thesis. Neither the thesis nor substantial extracts from it may be printed or otherwise reproduced without the author's permission.
AVIS:

L'auteur a accordé une licence non exclusive permettant à la Bibliothèque et Archives Canada de reproduire, publier, archiver, sauvegarder, conserver, transmettre au public par télécommunication ou par l'Internet, prêter, distribuer et vendre des thèses partout dans le monde, à des fins commerciales ou autres, sur support microforme, papier, électronique et/ou autres formats.

L'auteur conserve la propriété du droit d'auteur et des droits moraux qui protège cette thèse. $\mathrm{Ni}$ la thèse ni des extraits substantiels de celle-ci ne doivent être imprimés ou autrement reproduits sans son autorisation.
In compliance with the Canadian Privacy Act some supporting forms may have been removed from this thesis.

While these forms may be included in the document page count, their removal does not represent any loss of content from the thesis.
Conformément à la loi canadienne sur la protection de la vie privée, quelques formulaires secondaires ont été enlevés de cette thèse.

Bien que ces formulaires aient inclus dans la pagination, il n'y aura aucun contenu manquant.

\section{Canada}




\begin{abstract}
The concept of impedance transformation through gyrator-capacitor feedback circuit is investigated with the construction of an "active-inductor"-capacitor voltage controlled oscillator (or active LC-VCO) circuit. Insights on the active LC-VCO operation mechanism is then compared with a regular LC-VCO (or passive LC-VCO) and a divide-by-2 circuit. Both active and passive inductor-capacitor voltage controlled oscillator circuits are design, fabricated, and tested for this research.

Measurements on both active and passive LC-VCO circuits are close to their corresponding simulation results, with the exception of both oscillator circuits' phase noise performance. The passive LC-VCO circuit has a frequency span of $+/-7.9 \%$ from the centre frequency of $4.9675 \mathrm{GHz}$; and best phase noise measurements of $-66.72 \mathrm{dBc} / \mathrm{Hz}$ and $-74.37 \mathrm{dBc} / \mathrm{Hz}$ at $100 \mathrm{kHz}$ and $1 \mathrm{MHz}$ away from the oscillation frequency of $5.24 \mathrm{GHz}$. The active LC-VCO circuit has a frequency span of $+/-22.16 \%$ from the centre frequency of $4.2295 \mathrm{GHz}$; and best phase noise measurements of $63.62 \mathrm{dBc} / \mathrm{Hz}$ and $-66.8 \mathrm{dBc} / \mathrm{Hz}$ at $100 \mathrm{kHz}$ and $1 \mathrm{MHz}$ away from the oscillation frequency of $4.515 \mathrm{GHz}$.

Potential root causes of discrepancies between simulated and measured results include: singleended phase noise measurement from differential buffer output nodes, incomplete parasitic modeling for simulations, lack of on-chip decoupling capacitors for on-chip power and ground buses, and inaccurate modeling for frequency tuning components of both active and passive LC-VCO circuits; namely the voltage-to-current converter circuit and the PNP varactor devices.
\end{abstract}




\section{Acknowledgments}

I am grateful for the academic advice, patience, and encouragement provided by my thesis supervisor, Dr. James Wight. His guidance and knowledge have made it possible for me to complete my part-time master program.

I would like to thank Dr. Calvin Plett, for his technical advice throughout my thesis research. His generosity in sharing his expertise (in the area of RF and oscillator circuit deign) has enriched my learning experience as a graduate student.

I would also like to thank Dr. Gary Tarr and his research team, for their help on my circuit test board fabrication. Dr. Tarr's suggestion of doping gold layer onto glass substrate has made this test board design a reality.

In addition, I would like to acknowledge the Department of Electronics at Carleton University and thank all my graduate course lecturers; who have broaden my knowledge on integrated circuit design and related technical areas.

Furthermore, I would like to thank for all the support from my Synopsys (Mississauga office) managers, John Wilby, Duljit Malhi, Dino Toffolon, and the assistance from my co-workers in the product-test department, Adam Burns, and Scott Emery. Their help on my circuit testing allows me to complete my research with validated results from silicon. 
On the personal side, I would like to thank my friends/peers: Euhan Chong, John Danson, Dan Dinu, Neric Fong, Nelson Lam, Zhinian Shu, Thomas Wong, and Reza Yousefi; for sharing their technical knowledge with me.

Last, but not least, I would like to thank my family members for their patience and support. 


\section{Table of Contents}

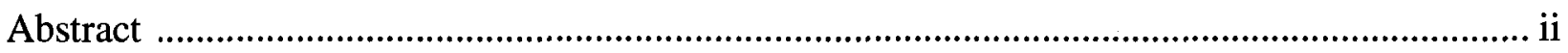

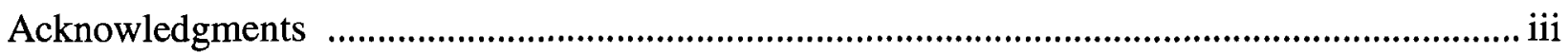

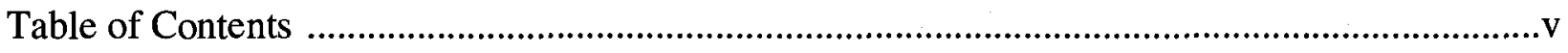

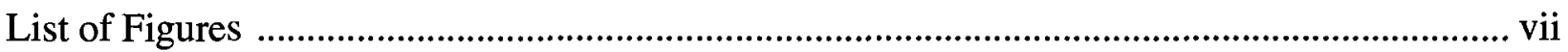

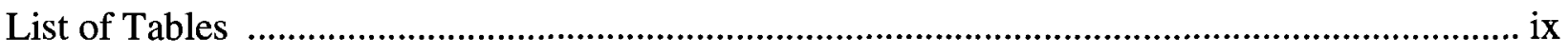

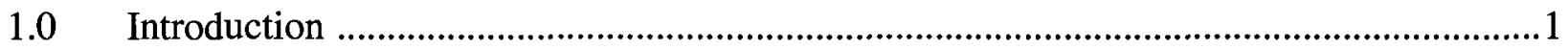

$1.1 \quad$ Research Motivation ....................................................................................

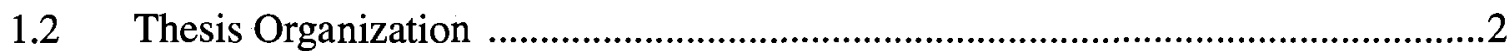

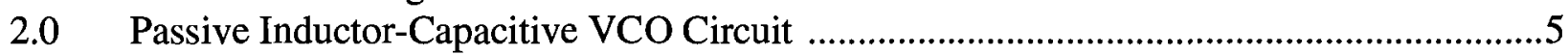

2.1 Passive Inductive-Capacitive "Tuned" Load ……….................................................6

2.1.1 Quality Factor of Inductor and Capacitor .....................................................6

2.1.2 Impact of Quality Factor on a "Tuned" Load .........................................12

2.1.3 On-chip Differential Spiral Inductor And ASITIC Model .........................19

2.1.4 PN-junction Varactor (lateral PNP bipolar transistor) .................................24

2.2 Active Components Of The "Tuned" Oscillator Circuit .........................................29

2.2.1 Complementary Negative-gm Gain Stage …………..................................29

2.2.2 Passive LC-VCO Buffer Stage ..................................................................31

2.3 Passive LC-VCO circuit operation mechanism ........................................................33

2.3.1 Complementary LC-VCO circuit topology ..............................................34

2.3.2 Complementary negative-gm LC-VCO operation mechanism ..................35

$2.4 \quad$ Passive LC-VCO noise sources ………….........................................................41

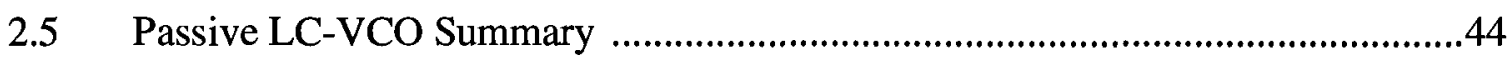

3.0 Active Inductor-Capacitor Voltage Controlled Oscillator .................................................45

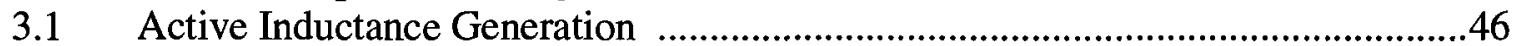

3.2 Implementation Of Active Inductance Generation ............................................50

3.2.1" Common-drain, Common-source" feedback loop ...................................52

3.2.2 “ Common-gate, Common-source" feedback loop ..........................................59

3.2.3“ Common-source, Common-source" feedback loop .....................................65

3.2.4 Summary of "gm" and "-gm" implementation in CMOS ............................70

3.3 Active-inductor-capacitor voltage controlled oscillator …….................................72

3.3.1 Implementation of an active-inductor based oscillator circuit ...................75

3.3.2 Bias Circuit Topology ........................................................................76

3.3.3 Voltage-to-Current Converter Circuit Topology ...........................................78

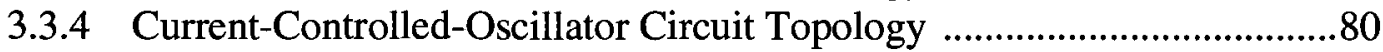

3.3.5 Output Buffer Circuit Topology ..........................................................82

3.3.6 Similarity with frequency divider circuit .............................................8

3.4 Active LC-VCO circuit operation mechanism ...................................................85

3.4.1 Frequency tuning of current controlled oscillator (small signal analysis) 87 
3.4.2 Frequency tuning of current controlled oscillator (large signal analysis) 88

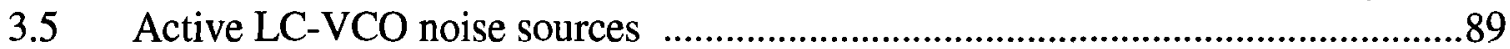

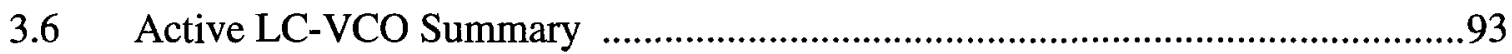

4.0 Oscillator Circuit Layout ..........................................................................................95

4.1 Passive-Inductor-Capacitor VCO Circuit Layout ....................................................95

4.1.1 On-chip Differential Spiral Inductor Layout ..............................................96

4.1.2 PN-junction Varactor (lateral PNP bipolar transistor) .................................97

4.1.3 Complementary Negative-gm Gain Stage ................................................99

4.1.4 Passive LC-VCO Buffer Stage ..........................................................101

4.1.5 Passive LC-VCO Circuit Layout (Top Level) ...........................................103

4.2 Active-Inductor-Capacitor VCO Circuit Layout ...................................................105

4.2.1 NMOS Current Mirror Circuit Layout ........................................................105

4.2.2 Voltage-to-Current Converter Circuit Layout ..........................................107

4.2.3 Current-controlled-oscillator Circuit Layout ..........................................108

4.2.4 Active LC-VCO Buffer Stage ..............................................................109

4.2.5 Active LC-VCO Circuits Layout ......................................................110

$4.3 \quad$ Top Level Circuit Layout …………………..................................................111

4.4 Off-Chip Circuit Test Board ..............................................................................115

4.4.1 Active LC-VCO Test Site And Wirebonding Diagram ............................116

4.4.2 Passive LC-VCO Test Site And Wirebonding Diagram ...........................118

4.5 Lab Test Equipment .....................................................................................119

4.6 Circuit Layout Summary ……...................................................................120

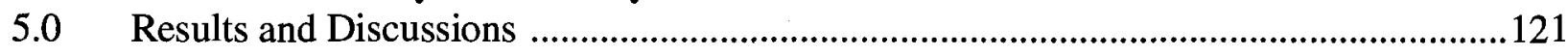

$5.1 \quad$ Phase noise and existing models ..................................................................122

5.1.1 Leeson's phase noise model ..............................................................123

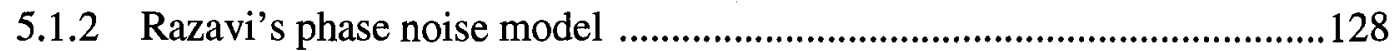

5.1.3 Hajimiri's phase noise model (for LC-VCO) ........................................132

5.1.4 Hajimiri's phase noise model (for ring oscillator) ..................................142

$5.2 \quad$ Performance of Active LC-VCO ........................................................................152

5.2.1 Impact on phase noise: choice of load .................................................157

5.2.2 Impact on phase noise: number of delay stages and flicker noise up-conver-

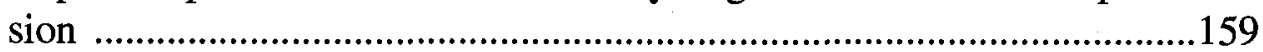

5.3 Performance of the Passive LC-VCO ...........................................................160

5.3.1 Impact on phase noise: asymmetrical rise and fall times ..........................164

5.3.2 Impact on phase noise: tail current bias device .....................................167

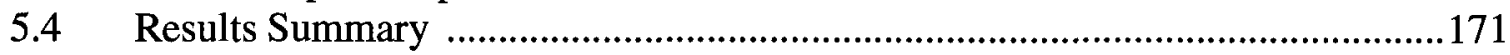

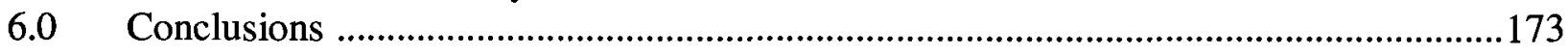

$6.1 \quad$ Summary Of Key Findings ............................................................................173

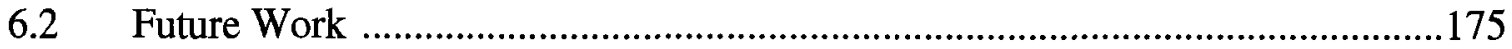




\section{List of Figures}

Figure 2.1 Conversion of series connection to shunt connection .............................................

Figure 2.2 Formation of a non-ideal LC-tank circuit model ................................................16

Figure 2.3 On-chip differential spiral inductor model (with ASITIC) ......................................21

Figure 2.4 Lateral PNP bipolar transistor as varactor (not to scale) ........................................

Figure 2.5 Schematic of complementary negative-gm gain stage (with MOS sizes) ..............31

Figure 2.6 Schematic of passive LC-VCO buffer circuit (with MOS sizes) ............................33

Figure 2.7 Similarity between a complementary negative-gm LC-VCO and a 2-stage tuned amplifier (in positive feedback configuration) ....................................................35

Figure 2.8 An illustration of a positive feedback loop with forward loop gain transfer function

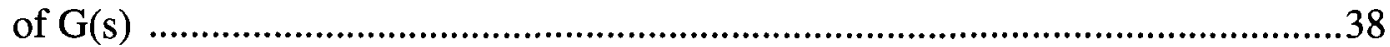

Figure 2.9 Complementary negative-gm LC-VCO with noise sources ...................................41

Figure 3.1 gyrator-capacitor circuit and its small signal models .............................................47

Figure 3.2 tank circuit resulted from active inductance circuit .................................................49

Figure 2.3 MOSFET (NMOS) device and its small signal model ........................................51

Figure 3.4 Bipolar (NPN) device and its small signal model ...............................................52

Figure 3.5 common-drain/common-source feedback configuration and its small signal models

Figure 3.6 common-gate/common-source feedback configuration and its small signal models

Figure 3.7 common-source/common-source feedback configuration and its small signal differ-

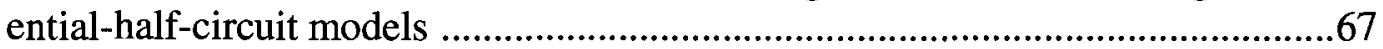

Figure 3.8 Conceptual active LC-VCO circuit and its tunable inductor model ......................74

Figure 3.9 schematic of active LC-VCO circuit with bias and frequency tuning circuits .....76

Figure 3.10

Figure 3.11

Figure 3.12

Figure 3.13

Figure 3.14

Figure 3.15

Figure 3.16

Figure 4.1

Figure 4.2

Figure 4.3

Figure 4.4

Figure 4.5

Figure 4.6

Figure 4.7

Figure 4.8

Figure 4.9

Figure 4.10

Figure 4.11

Figure 5.1

Figure 5.2

active LC-VCO circuit output frequency tuning (bias scheme) ................................79

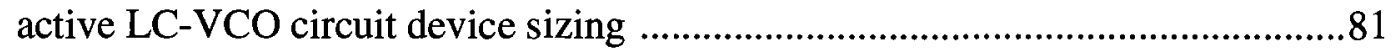

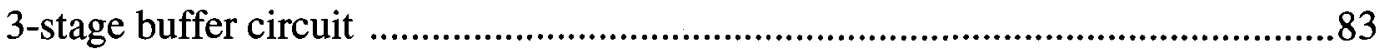

schematic of a divided-by-2 circuit with top level and transistor level views ......84

ring oscillator model with $\mathrm{N}$ stages of single-ended inverters ................................86

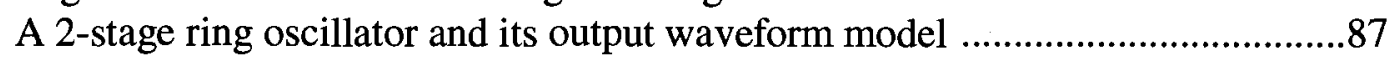

On-chip differential spiral inductor layout (not to scale) ........................................96

Layout of lateral PNP bipolar transistor as varactor (not to scale) ........................98

Layout of complementary negative-gm gain stage (not to scale) ........................100

Layout of buffer for passive LC-VCO circuit (not to scale) .................................103

Layout of passive LC-VCO circuit (top level, not to scale) .................................104

Layout of active LC-VCO circuit (not to scale) .................................................111

Layout of active LC-VCO circuit (2nd version, not to scale) ..............................114

gold plated 2"x2" test structure on glass (to scale) ………………………...........116

Active LC-VCO test site and wirebonding diagram ………….............................117

Passive LC-VCO test site and wirebonding diagram .........................................118

oscillators under tests ....................................................................................120

Power Spectral Densities at oscillator input and output ......................................124

Razavi's linear closed-loop oscillator model ....................................................129 
Figure 5.3 Impulse Sensitivity Function of an LC-VCO .............................................133

Figure 5.4 Relationship between LC-VCO output voltage and its ISF waveforms .............135

Figure 5.5 Impulse Sensitivity Function of a ring oscillator circuit .................................143

Figure 5.6 Relationship between ring oscillator normalized output voltage and its ISF wave-

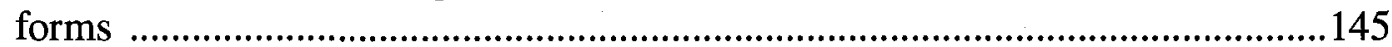

Figure 5.7 Relationship between ring oscillator circuit output signal rise time and delay ...147

Figure 5.8 Simulated results of active LC-VCO frequency tuning .....................................153

Figure 5.9 Simulated results of passive LC-VCO frequency tuning ..................................161

Figure 5.10 Up-conversion of flicker noise and down-conversion of noises in even order harmonics vicinity onto oscillator phase noise ..................................................168

Figure 5.11 Complementary LC-VCO node voltages and effect of shunt capacitor "Cbias" on bias NMOS Vds ripple reduction ..................................................................169

Figure 5.12 Cross-coupled NMOS device duty cycle reduction effect from shunt capacitor

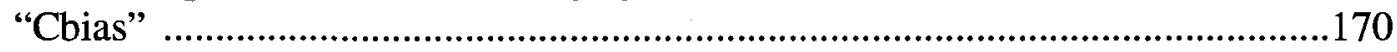




\section{List of Tables}

Table 2.1 ASITIC characterization of an on-chip passive differential spiral inductor ..............22

Table 2.2 Frequency tuning with 8 shunt connected PNP varactors .......................................28

Table 3.1 Summary of "gm" and "-gm" implementation in CMOS technology .......................71

Table 5.1 Post layout simulation results and lab measurements of active LC-VCO circuit .....154

Table 5.2 Post layout simulation results and lab measurements of passive LC-VCO circuit ...162 


\section{CHAPTER 1 Introduction}

\subsection{Research Motivation}

Relaxation and resonator based oscillators are two of the most popular circuit topologies chosen for voltage controlled oscillator implementations. Relaxation oscillator circuits provide two major advantages including wider frequency tuning range and smaller circuit area; however, these circuits suffer relatively poorer performances (i.e. higher phase noise in output spectrum) when compared with resonator based oscillator circuits. Resonator based oscillator circuits, on the other hand, require relatively larger circuit area. In addition, the resonators have been commonly implemented off-chip in the past. As 
semiconductor fabrication technology advances, on-chip implemenation of both resonator and oscillator circuits become a viable option. Concurrently, research efforts have been made on further reduction of overall circuit area. One key research topic focus on active inductance generation by means of impedance transformation.

The principle of impedance transformation with gyrator circuits has been proposed over the past few decades [2]. The gyrator circuit "transforms" the impedance of an lumped element into another element with different characteristics. Specifically, we are interested in applying this transformation property in order to convert a capacitive load into an on-chip inductive load.

The aim of this research is to investigate the application of gyrator-capacitor-based active inductance generation onto oscillator circuits. Both relaxation and resonator based oscillator circuits are built in TSMC180nm technology.

\subsection{Thesis Organization}

This thesis is organized in 6 chapters, and contents of each chapter are briefly summarized as follows: 
Chapter 2 covers both theory and technical background information related to resonator type of oscillator circuit. Specifically, an integrated voltage controlled oscillator circuit was implemented with on-chip spiral inductor and varactors.

Chapter 3 covers both theory and technical background information related to the principle of active inductance generation from gyrator-capactive feedback circuits, and the proposed relaxation type of voltage controlled oscillator circuit.

Chapter 4 covers the circuit layout implementation of both voltage controlled circuits introduced in Chapters 2 and 3. In addition, layout for the off-chip test structure is also covered in this chapter.

Chapter 5 provides a few examples of existing phase noise models, simulation and measurement results of proposed oscillator circuits, and discussions on performance of the implemented voltage controlled circuits.

Chapter 6 concludes the thesis with a summary of findings and possible future work. 


\section{CHAPTER 2 Passive Inductor- Capacitive VCO Circuit}

This chapter covers the design of a passive inductor-capacitive voltage controlled oscillator circuit (or simply LC-VCO). The oscillator core circuit consists of a narrowband "tuned" load, and a cross-coupled negative "gm" gain stage. Since we have to drive the signal off-chip, a buffer circuit is added in between the LC-VCO core circuit and the output pads.

The definition of quality factor and descriptions on the design of the passive components will be covered in the first half of this chapter. Descriptions on the design of the active circuit components and the operation principle of the implemented tuned-oscillator circuit will be covered in the second half of this chapter. 


\subsection{Passive Inductive-Capacitive "Tuned" Load}

The "tuned" load of an oscillator core circuit consists of an on-chip differential spiral inductor, connected in parallel with a capacitor. In order to vary the oscillation frequency of this oscillator (i.e. as a voltage controlled oscillator), the inductor and/or the capacitor is designed to be tunable. In this research, the capacitor is made tunable; and is referred by the name "varactor" (i.e. variable capacitor). In addition, the inductor is designed to have a constant value; and is implemented with an on-chip spiral inductor of fixed dimension.

\subsubsection{Quality Factor of Inductor and Capacitor}

The quality factor (or Q-factor) is used to determine how close the implementation of an inductor or a capacitor is to its ideal value. The non-ideal factor in such implementation arises from actual physical properties of the passive component material. 
In the case of the on-chip spiral inductor, it is composed of a piece of metal line routed in a spiral form. The non-ideal factor derives from the non-zero resistivity along this metal routing, and associated routing to other circuit components.

Similarly for the varactor implementation, series resistivity along metal line routing and inside n-wells are factors that degrade the quality factor as well. More information on quality factor degradation will be described in later sections.

By definition, the quality factor of an inductor with a series-connected resistor is

$$
Q=\frac{\operatorname{Im}\left(Z_{L}\right)}{\operatorname{Re}\left(Z_{L}\right)}=\frac{\omega \cdot L_{s}}{R_{s}}
$$

where the subscript "s" represents series-connection between the inductor and the resistor.

Similarly, the quality factor of a capacitor with a series-connected resistor is

$$
Q=\frac{\operatorname{Im}\left(Z_{C}\right)}{\operatorname{Re}\left(Z_{C}\right)}=\frac{\frac{1}{\omega \cdot C_{s}}}{R_{s}}=\frac{1}{\omega \cdot R_{s} \cdot C_{s}}
$$

where the subscript "s" represents series-connection between the capacitor and the resistor. 
For simplicity on estimation of the quality factor of a LC shunt-connected load, the series-connected resistive-inductive and/or resistive-capacitive model is represented by an equivalent shunt-connected model. The conversion (from a series-connected resistive model to a shunt-connected resistive model) is illustrated in Figure 2.1.
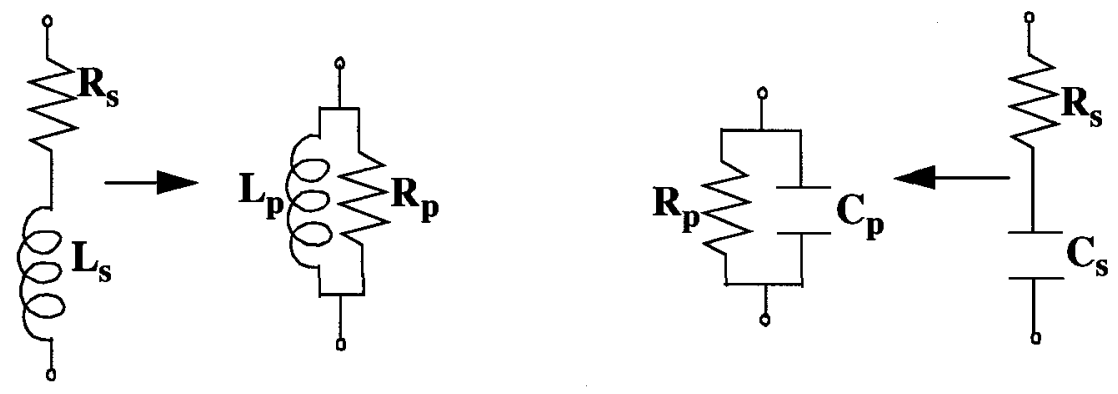

Figure 2.1: Conversion of series connection to shunt connection

Since the impedance of an inductor and/or capacitor varies with frequency, conversion between series/shunt-connection types can only be applied at a specific frequency (i.e. narrow band). The derivation of the formulas can be found in references [8] and [22].

For the case of a series-connected inductor with resistor:

$$
s \cdot L_{s}+R_{s}=Z_{e q}=\frac{s \cdot L_{p} \cdot R_{p}}{s \cdot L_{p}+R_{p}}
$$


where subscript "s" and "p" represents series-connection and shunt-connection respectively.

After applying cross-multiplication and substitution of variable "s $=\mathrm{j} \omega$ ":

$$
-\omega^{2} \cdot L_{s} \cdot L_{p}+j \omega \cdot\left(L_{s} \cdot R_{p}+L_{p} \cdot R_{s}\right)+R_{s} \cdot R_{p}=j \omega \cdot L_{p} \cdot R_{p}
$$

After equating real and imaginary parts:

$$
\begin{gathered}
j \omega \cdot\left(L_{s} \cdot R_{p}+L_{p} \cdot R_{s}\right)=j \omega \cdot\left(L_{p} \cdot R_{p}\right) \\
-\omega^{2} \cdot L_{s} \cdot L_{p}+R_{s} \cdot R_{p}=0
\end{gathered}
$$

After isolating $R_{p}$ in Equation 2.6 and substituting back to Equation 2.5:

$$
\frac{\omega^{2} \cdot L_{s} \cdot L_{p}^{2}}{R_{s}}=L_{p} \cdot\left(R_{s}+\frac{\omega^{2} \cdot L_{s}^{2}}{R_{s}}\right)
$$

After isolating $\mathrm{L}_{\mathrm{p}}$ in Equation 2.7 and re-arranging the terms:

$$
L_{p}=\left(\frac{R_{s}}{\omega^{2} \cdot L_{s}}\right) \cdot\left(R_{s}+\frac{\omega^{2} \cdot L_{s}{ }^{2}}{R_{s}}\right)=L_{s} \cdot\left(\frac{R_{s}{ }^{2}}{\omega^{2} \cdot L_{s}{ }^{2}}+1\right)=L_{s} \cdot\left(1+\frac{1}{Q^{2}}\right)=L_{s} \cdot\left(\frac{Q^{2}+1}{Q^{2}}\right)
$$


where " $Q$ " is the quality factor of an inductor with a series-connected resistor; and is shown in Equation 2.1.

Substituting Equation 2.8 back to Equation 2.6, and calculate for $R_{p}$ :

$$
R_{p}=\frac{1}{R_{s}} \cdot \omega^{2} \cdot L_{s}^{2} \cdot\left(1+\frac{1}{Q^{2}}\right)=R_{s} \cdot Q^{2} \cdot\left(1+\frac{1}{Q^{2}}\right)=R_{s} \cdot\left(1+Q^{2}\right)
$$

For $\mathrm{Q}$ of large values, $\mathrm{L}_{\mathrm{p}}$ and $\mathrm{R}_{\mathrm{p}}$ can be approximated to $\mathrm{L}_{\mathrm{p}}=\mathrm{L}_{\mathrm{s}}$ and $\mathrm{R}_{\mathrm{p}}=\mathrm{R}_{\mathrm{s}}{ }^{*} \mathrm{Q}^{2}$ respectively.

For the case of a series-connected capacitor with resistor:

$$
\frac{1}{s \cdot C_{s}}+R_{s}=\frac{1+s \cdot R_{s} \cdot C_{s}}{s \cdot C_{s}}=Z_{e q}=\frac{R_{p} \cdot \frac{1}{s \cdot C_{p}}}{R_{p}+\frac{1}{s \cdot C_{p}}}=\frac{R_{p}}{1+s \cdot R_{p} \cdot C_{p}}
$$

where subscript " $\mathrm{s}$ " and " $\mathrm{p}$ " represents series-connection and shunt-connection respectively.

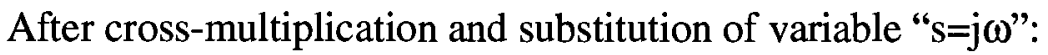

$$
1+j \omega \cdot\left(R_{s} \cdot C_{s}+R_{p} \cdot C_{p}\right)-\left(\omega^{2} \cdot R_{s} \cdot C_{s} \cdot R_{p} \cdot C_{p}\right)=j \omega \cdot R_{p} \cdot C_{s}
$$


After equating real and imaginary parts:

$$
\begin{gathered}
1-\left(\omega^{2} \cdot R_{s} \cdot R_{p} \cdot C_{s} \cdot C_{p}\right)=0 \\
\left(R_{s} \cdot C_{s}+R_{p} \cdot C_{p}\right)=R_{p} \cdot C_{s}
\end{gathered}
$$

After isolating $R_{p}$ in Equation 2.12, and substituting into Equation 2.13:

$$
\left(R_{s} \cdot C_{s}+\frac{1}{\omega^{2} \cdot R_{s} \cdot C_{s}}\right)=\left(\frac{1+\omega^{2} \cdot R_{s}{ }^{2} \cdot C_{s}^{2}}{\omega^{2} \cdot R_{s} \cdot C_{s}}\right)=\frac{1}{\omega^{2} \cdot R_{s} \cdot C_{p}}
$$

After simplifying Equation 2.14, and re-arranging the terms:

$$
C_{p}=\frac{C_{s}}{1+\omega^{2} \cdot R_{s}{ }^{2} \cdot C_{s}{ }^{2}}=\frac{C_{s}}{1+\frac{1}{Q^{2}}}=C_{s} \cdot\left(\frac{Q^{2}}{Q^{2}+1}\right)
$$

where $\mathrm{Q}=1 /\left(\mathrm{s} * \mathrm{R}_{\mathrm{S}} * \mathrm{C}_{\mathrm{S}}\right)$ is the quality factor of a capacitor with a series-connected resistor.

Substituting Equation 2.15 back to Equation 2.12, and calculate for $\mathrm{R}_{\mathrm{p}}$ : 


$$
R_{p}=R_{s} \cdot \frac{C_{s}}{C_{s}-C_{p}}=R_{s} \cdot \frac{1}{1-\frac{C_{p}}{C_{s}}}=R_{s} \cdot \frac{1}{1-\left(\frac{Q^{2}}{1+Q^{2}}\right)}=R_{s} \cdot\left(1+Q^{2}\right)
$$

For large values of $\mathrm{Q}, \mathrm{C}_{\mathrm{p}}$ and $\mathrm{R}_{\mathrm{p}}$ can be approximated as $\mathrm{C}_{\mathrm{p}}=\mathrm{C}_{\mathrm{s}}$ and $\mathrm{R}_{\mathrm{p}}=\mathrm{R}_{\mathrm{s}} * \mathrm{Q}^{2}$ respectively.

\subsubsection{Impact of Quality Factor on a "Tuned" Load}

The impedance and admittance of an ideal LC-tank circuit (i.e. formed by shunt connection of an ideal inductor with an ideal capacitor) can be modelled as follows:

$$
\begin{gathered}
Z_{\text {ideal }-L C-\tan k}=\frac{\left(j \omega \cdot L_{p}\right) \cdot\left(\frac{1}{j \omega \cdot C_{p}}\right)}{\left(j \omega \cdot L_{p}\right)+\left(\frac{1}{j \omega \cdot C_{p}}\right)}=\frac{j \omega \cdot L_{p}}{1-\left(\omega^{2} \cdot L_{p} \cdot C_{p}\right)} \\
Y_{\text {ideal }-L C-\tan k}=\left(j \omega \cdot C_{p}\right)+\left(\frac{1}{j \omega \cdot L_{p}}\right)=j \cdot\left[\left(\omega \cdot C_{p}\right)-\left(\frac{1}{\omega \cdot L_{p}}\right)\right]
\end{gathered}
$$


From Equations 2.1, 2.2, and 2.10; the quality factor of such an ideal LC-tank circuit can be modelled as:

$$
Q_{\text {ideal }-L C-\tan k}=\frac{\operatorname{Im}\left(Z_{\text {ideal }-L C-\tan k}\right)}{\operatorname{Re}\left(Z_{\text {ideal }-L C-\tan k}\right)}=\frac{\omega \cdot L_{p}}{1-\left(\omega^{2} \cdot L_{p} \cdot C_{p}\right)} \cdot \frac{1-\left(\omega^{2} \cdot L_{p} \cdot C_{p}\right)}{0} \equiv I N F I N I T Y
$$

which is expected, as there is no resistive element (i.e. no real part of $Z_{\text {ideal-LC-tank }}$ ) found in such a circuit.

As frequency increases from a value lower than the resonant frequency, the capacitive component can be approximated as an open circuit, whereas the inductive component dominates the behaviour of the LC-tank circuit. This can be modelled as follows:

$$
\lim _{\omega \rightarrow 0} Y_{\text {ideal }-L C-\tan k}=\lim _{\omega \rightarrow 0}\left(j \omega \cdot C_{p}\right)+\lim _{\omega \rightarrow 0}\left(\frac{1}{j \omega \cdot L_{p}}\right) \approx 0+\lim _{\omega \rightarrow 0}\left(\frac{1}{j \omega \cdot L_{p}}\right)=Y_{L}
$$

and

$$
\angle Z_{\text {ideal }-L C-\tan k} \approx \angle Z_{\text {inductor }}=\angle\left(j \omega \cdot L_{p}\right)=90^{\circ}
$$


At resonant frequency

$$
f=\frac{\omega}{2 \cdot \pi}=\frac{1}{2 \cdot \pi \cdot \sqrt{L_{p} \cdot C_{p}}}
$$

the admittance approaches zero and the LC-tank circuit behaves like an open circuit:

$$
\lim _{\omega \rightarrow \frac{1}{\sqrt{L \cdot C}}} Y_{\text {ideal }-L C-\tan k}=\lim _{\omega \rightarrow \frac{1}{\sqrt{L \cdot C}}} j \cdot\left[\left(\omega \cdot C_{p}\right)-\left(\frac{1}{\omega \cdot L_{p}}\right)\right] \approx \frac{C_{p}}{\sqrt{L_{p} \cdot C_{p}}}-\frac{1}{\left(\frac{L_{p}}{\sqrt{L_{p} \cdot C_{p}}}\right)}=0
$$

As frequency increases to a value much higher than the resonant frequency, the inductive component can be approximated as an open circuit; whereas the capacitive component dominates the behaviour of the LC-tank circuit. This behaviour can be modelled as follows:

$$
\lim _{\omega \rightarrow \infty} Y_{\text {ideal }-L C-\tan k}=\lim _{\omega \rightarrow \infty}\left(j \omega \cdot C_{p}\right)+\lim _{\omega \rightarrow \infty}\left(\frac{1}{j \omega \cdot L_{p}}\right) \approx \lim _{\omega \rightarrow 0}\left(j \omega \cdot C_{p}\right)+0=Y_{C}
$$

and

$$
\angle Z_{\text {ideal }-L C-\tan k} \approx \angle Z_{\text {capacitor }}=\angle\left(\frac{1}{j \omega \cdot C_{p}}\right)=-90^{\circ}
$$


For a non-ideal case, all series-connected resistance along the inductor and capacitor can be converted to an equivalent shunt-connected value (i.e. as shown in the previous section). The resistive loss of a inductive-capacitive "tuned" load (or "LC-tank") can then be modelled as follows:

$$
R_{e q}=R_{p(L C-\tan k)}=\frac{R_{p(i n d)} \cdot R_{p(c a p)}}{R_{p(i n d)}+R_{p(c a p)}}=\frac{\left[R_{s(i n d)} \cdot\left(1+Q_{(i n d)}{ }^{2}\right)\right] \cdot\left[R_{s(c a p)} \cdot\left(1+Q_{(c a p)}{ }^{2}\right)\right]}{\left[R_{s(\text { ind })} \cdot\left(1+Q_{(i n d)}{ }^{2}\right)\right]+\left[R_{s(c a p)} \cdot\left(1+Q_{(c a p)}\right)\right]}
$$

where the subscripts "ind" and "cap" represent inductor and capacitor (inside the LC-tank circuit) respectively; and the values of both inductive and capacitive components are modelled as follows:

$$
\begin{gathered}
L_{p(L C-\tan k)}=L_{p(\text { inductor })}=L_{s} \cdot\left(\frac{R_{s}^{2}}{\omega^{2} \cdot L_{s}^{2}}+1\right)=L_{s} \cdot\left(1+\frac{1}{Q_{(\text {ind })}^{2}}\right) \\
C_{p(L C-\tan k)}=C_{p(\text { capacitor })}=\frac{C_{s}}{1+\frac{1}{Q_{(c a p)}^{2}}}=C_{s} \cdot\left(\frac{Q_{(\text {cap })}^{2}}{Q_{(\text {cap })}^{2}+1}\right)
\end{gathered}
$$


where $Q_{(\text {ind) }}$ and $Q_{\text {(cap) }}$ represent the quality factor of the inductor and capacitor respectively.

From equations Equations 2.26 and 2.27, ideal shunt-connected inductive and capacitive components inside a non-ideal LC-tank circuit are separated from their corresponding resistive elements. Thus the resistive loss of this LC-tank circuit can be formed by the two shunt-connected resistive models, which are derived from their corresponding non-ideal inductor and capacitor models (see Figure 2.2).

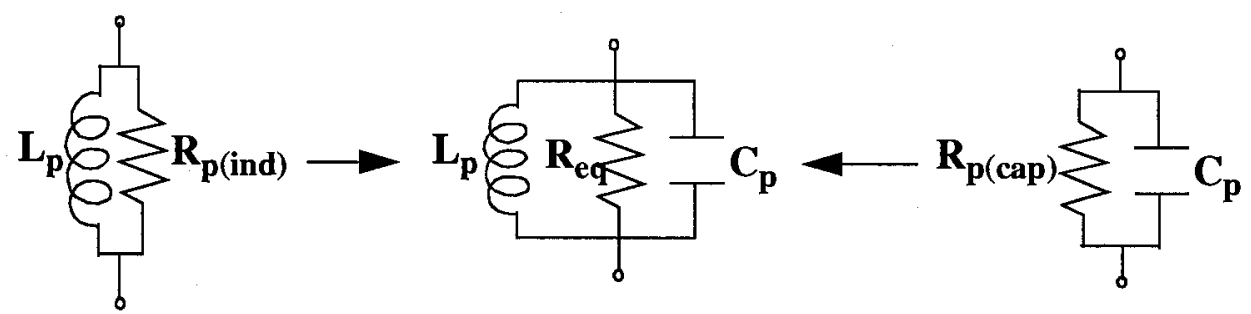

Figure 2.2: Formation of a non-ideal LC-tank circuit model

The impedance of such a non-ideal LC-tank circuit will then be $\mathrm{R}_{\mathrm{eq}}$ in parallel with the ideal shunt-connected inductor and capacitor (i.e. $\mathbf{R}_{\mathrm{eq}}$ is shunt-connected to an ideal LC-tank). 
Similar to the ideal LC-tank circuit, the impedance is dominated by the shunt-connected resistor and inductor, as frequency increases from zero. At resonant frequency, the ideal inducive and capacitive components form an open circuit, and the impedance of a non-ideal LC-tank circuit is equal to the shunt connected resistor $\mathrm{R}_{\mathrm{eq}}$. As frequency approaches infinity, the impedance is dominated by the shunt-connected resistor and capacitor. This can be illustrated as follows:

$$
Y_{L C-\tan k}=\frac{1}{R_{e q}}+\left(j \omega \cdot C_{p}\right)+\left(\frac{1}{j \omega \cdot L_{p}}\right)=j \cdot\left[\left(\omega \cdot C_{p}\right)-\left(\frac{1}{\omega \cdot L_{p}}\right)\right]+\frac{1}{R_{e q}}
$$

As frequency approaches zero:

$$
\lim _{\omega \rightarrow 0} Y_{L C-\tan k}=\lim _{\omega \rightarrow 0}\left(\frac{1}{R_{e q}}\right)+\lim _{\omega \rightarrow 0}\left(j \omega \cdot C_{p}\right)+\lim _{\omega \rightarrow 0}\left(\frac{1}{j \omega \cdot L_{p}}\right) \approx \frac{1}{R_{e q}}+0+\lim _{\omega \rightarrow 0}\left(\frac{1}{j \omega \cdot L_{p}}\right)=\frac{1}{R_{e q}}+Y
$$

As frequency approaches the resonant frequency: 
$\lim _{\omega \rightarrow \frac{1}{\sqrt{L \cdot C}}} Y_{L C-\tan k}=\lim _{\omega \rightarrow \frac{1}{\sqrt{L \cdot C}}} j \cdot\left[\left(\omega \cdot C_{p}\right)-\left(\frac{1}{\omega \cdot L_{p}}\right)\right]+\frac{1}{R_{e q}} \approx \frac{1}{R_{e q}}+\frac{C_{p}}{\sqrt{L_{p} \cdot C_{p}}}-\frac{1}{\left(\frac{L_{p}}{\sqrt{L_{p} \cdot C_{p}}}\right)}=\frac{1}{R_{e q}}$

and

$$
\angle Z_{L C-\tan k} \approx \angle Z_{\text {resistor }}=0^{\circ}
$$

As frequency approaches infinity:

$\lim _{\omega \rightarrow \infty} Y_{L C-\tan k}=\lim _{\omega \rightarrow 0}\left(\frac{1}{R_{e q}}\right)+\lim _{\omega \rightarrow \infty}\left(j \omega \cdot C_{p}\right)+\lim _{\omega \rightarrow \infty}\left(\frac{1}{j \omega \cdot L_{p}}\right) \approx \lim _{\omega \rightarrow 0}\left(j \omega \cdot C_{p}\right)+0+\frac{1}{R_{e q}}=\frac{1}{R_{e q}}+Y_{C}$

Hence we have proven with above equations, that the quality factor degradation of a LC-tank tuned load can be modelled as a resistor connected in parallel to an ideal tuned load of same reactive (i.e. inductive and capacitive) values. Alternatively, one can interpret the shunt connected resistor as having a value of infinity in an ideal LC-tank circuit case; and the circuit becomes an open circuit at resonant frequency. 


\subsubsection{On-chip Differential Spiral Inductor And ASITIC Model}

As described in Dr. Niknejad's inductor/transformer textbook [12], there are a few contributing factors to the quality factor degradation of an on-chip inductor, and are generally described as "losses".

The first type of loss is related to resistance inside the metal routing of the on-chip spiral inductor. For an input signal with low frequency content (i.e. including DC voltage), the value is quantified and supplied by the semiconductor foundry as "sheet resistivity". As the frequency of the input signal increases, current tends to flow at edges of the metal routing; thus the effective metal thickness is reduced, and resistivity increases. The effect is modeled as "skin effect". In order to reduce this type of loss, semiconductor foundries provide a relatively thicker layer of metal at the top of the process.

The second type of loss is based on the parasitic capacitance between the inductor metal routing and the lossy substrate material underneath it. This "capacitive" value decreases as the distance between the inductor and substrate increases, hence on-chip spiral inductors are usually implemented with the top two or three metal layers only. In addi- 
tion, this "capacitive" value also decreases with the area of the on-chip spiral inductor; thus a differential on-chip spiral inductor is implemented for this research project.

The third type of loss is related to magnetic coupling between the on-chip spiral inductor structure and the substrate underneath it. This behaviour can be modelled by "Faraday's Law" and "Lenz's Law". As an applied current flows through the spiral inductor in a particular direction for half of the oscillation period, and in the opposite direction for the other half of the period; a magnetic flux is generated and passes through the substrate. From this magnetic flux, an "eddy" current is induced inside the lossy substrate. Hence this substrate resistance becomes shunt-connected to the spiral inductor through magnetic coupling; and the spiral inductor quality factor is degraded.

The physical dimension and the narrow-band model of the on-chip differential spiral inductor have been defined and optimized by ASITIC; which is a CAD tool designed for on-chip inductor and transformer modeling (by Dr. Niknejad). The dimension of the inductor layout is illustrated in the layout section, whereas the inductor model (see Figure 2.3) is optimized and summarized as follows: 


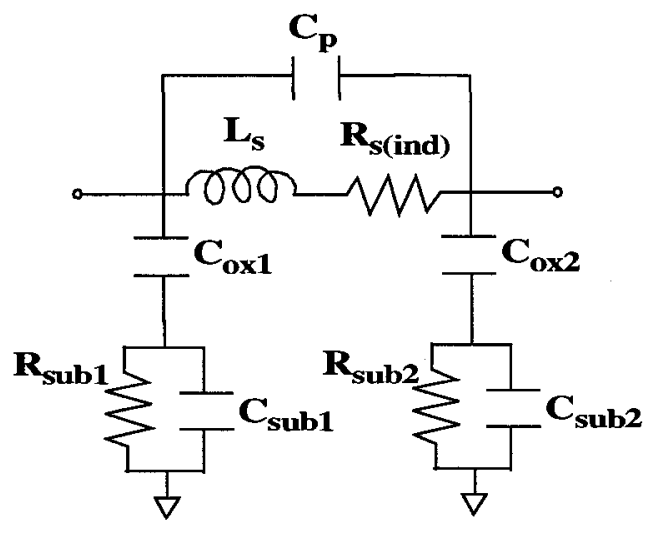

Figure 2.3: On-chip differential spiral inductor model (with ASITIC)

The above model is composed of a few passive components:

- $\mathrm{C}_{\mathrm{p}}$ represents the parasitic "metal-insulator-metal" capacitance between two ends of the on-chip spiral inductor (i.e. top-metal layer and "underpass" layer)

- $\mathrm{L}_{\mathrm{s}}$ represents the inductance along the spiral inductor metal routing, whereas $\mathrm{R}_{\mathrm{s}}$ represents the associated loss; hence these two components are modelled with a series connection

- $\mathrm{C}_{\mathrm{ox} 1}$ and $\mathrm{C}_{\mathrm{ox} 2}$ represent parasitic capacitance between the inductor metal routing and the substrate underneath it 
- $\mathrm{R}_{\text {sub1 }}$ and $\mathrm{R}_{\text {sub2 }}$ represent losses within the substrate material, whereas $\mathrm{C}_{\text {sub1 }}$ and $\mathrm{C}_{\text {sub2 }}$ represent parasitic capacitance between the top and bottom surfaces of the substrate material

The model provided by ASITIC is a narrow-band approximation, and simulations were performed across a wide range of frequencies in order to obtain a better understanding on the inductor performance over these frequencies. The values for $C_{p}, C_{o x 1}$, $\mathrm{C}_{\mathrm{ox} 2}$ are evaluated to be $18 \mathrm{fF}, 100 \mathrm{fF}$, and $100 \mathrm{fF}$ respectively, and the values for other passive comonents are summarized as follows:

TABLE 2.1. ASITIC characterization of an on-chip passive differential spiral inductor

\begin{tabular}{lllllllll}
\hline $\begin{array}{l}\text { Freq_in } \\
(\mathbf{G H z})\end{array}$ & $\mathbf{Q}$ & $\mathbf{L}_{\mathbf{s}}(\mathbf{n H})$ & $\begin{array}{l}\mathbf{R}_{\mathbf{s}} \\
(\mathbf{O h m})\end{array}$ & $\begin{array}{l}\mathbf{C s u b}_{1} \\
(\mathbf{f F})\end{array}$ & $\begin{array}{l}\mathbf{R s u b}_{1} \\
(\mathbf{O h m})\end{array}$ & $\begin{array}{l}\mathbf{C s u b}_{2} \\
(\mathbf{f F})\end{array}$ & $\begin{array}{l}\mathbf{R s u b}_{2} \\
(\mathbf{O h m})\end{array}$ & $\begin{array}{l}\text { Freq_Sr } \\
(\mathbf{G H z})\end{array}$ \\
2.0 & 4.2 & 1.95 & 5.56 & 61.7 & 856 & 57.3 & 983 & 14.49 \\
2.5 & 4.89 & 1.95 & 5.77 & 58.7 & 846 & 54.6 & 974 & 14.86 \\
3.0 & 5.44 & 1.96 & 5.96 & 55.5 & 835 & 51.7 & 963 & 15.27 \\
3.5 & 5.89 & 1.96 & 6.14 & 52.3 & 821 & 48.8 & 951 & 15.71 \\
4.0 & 6.24 & 1.97 & 6.31 & 49.1 & 806 & 45.8 & 938 & 16.71 \\
4.5 & 6.51 & 1.99 & 6.45 & 46.1 & 791 & 42.9 & 923 & 16.63 \\
5.0 & 6.69 & 2.00 & 6.58 & 43.2 & 774 & 40.2 & 907 & 17.1 \\
5.5 & 6.8 & 2.02 & 6.7 & 40.6 & 756 & 37.6 & 890 & 17.56 \\
6.0 & 6.86 & 2.05 & 6.82 & 38.1 & 738 & 35.2 & 872 & 18.01 \\
6.5 & 6.85 & 2.08 & 6.93 & 35.9 & 719 & 33.1 & 853 & 18.43 \\
7.0 & 6.79 & 2.11 & 7.03 & 33.9 & 700 & 31.1 & 833 & 18.82 \\
7.5 & 6.69 & 2.15 & 7.14 & 32.1 & 681 & 29.3 & 813 & 19.19 \\
8.0 & 6.54 & 2.19 & 7.25 & 30.4 & 661 & 27.7 & 792 & 19.51
\end{tabular}


where "Freq_sr" is the self-resonant frequency of the on-chip spiral inductor.

At this "self-resonant" frequency, the reactive components (i.e. series inductance and parasitic capacitance) cancel each other; and the structure oscillates. Since only the parasitic capacitance is considered for self-resonant frequency, any additional capacitance to the on-chip spiral inductor structure will only further reduce the oscillation frequency. In other words, the self-resonant is the highest possible oscillation frequency that can be attained with this on-chip spiral inductor implementation.

From the inductor characterization data (summarized in Table 1), the quality factor of the on-chip spiral inductor peaks between $5.5 \mathrm{GHz}$ and $6 \mathrm{GHz}$. Such behaviour of the inductor model can be explained as follows:

At low frequency, the impedance of the series resistor $R_{s}$ dominates over the impedance of the series inductor $\mathrm{L}_{\mathrm{s}}$; hence the quality factor is relatively low. At high frequency, where impedances of $\mathrm{C}_{\mathrm{ox} 1}$ and $\mathrm{C}_{\mathrm{ox} 2}$ are much smaller than the impedances of $\mathrm{C}_{\text {sub1 }}$ and $\mathrm{C}_{\text {sub2 }}$, the impedance of resistors $\mathrm{R}_{\text {sub1 }}$ and $\mathrm{R}_{\text {sub2 }}$ are shunt connected directly to the two ends of the inductor model; thus the quality factor drops as well. 


\subsubsection{PN-junction Varactor (lateral PNP bipolar transistor)}

Accumulation mode MOS devices and pn-junction based varactors have been commonly found in many LC-VCO design/research papers. Since the pn-junction based structure was the only type of varactor model available in our chosen TSMC 180um CMOS technology, the varactor was thus implemented with lateral PNP bipolar devices (see Figure 2.4).

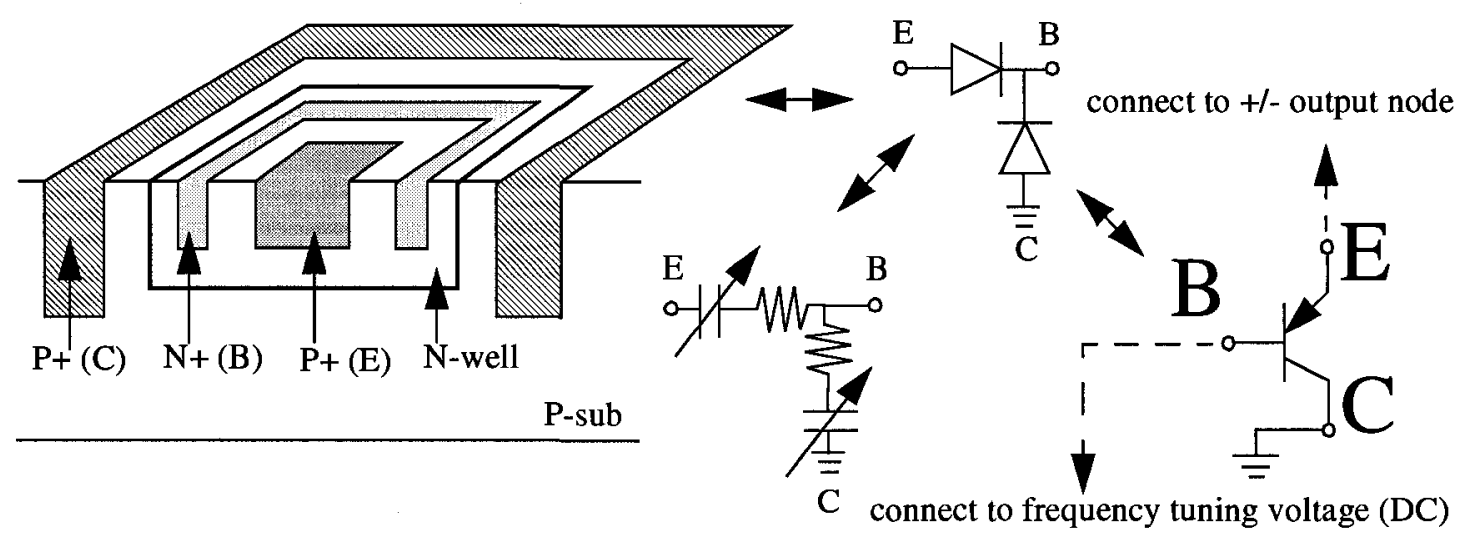

Figure 2.4: Lateral PNP bipolar transistor as varactor (not to scale)

As illustrated in Figure 2.4, the frequency tuning DC voltage is applied at the base (B) node of the varactor structure; which also acts as an "AC ground" for the oscillation 
signal. Thus the junction capacitance between the emitter (E) and base (B) nodes is used to vary the oscillation frequency, whereas the junction capacitance between the base (B) and collector (C) nodes is isolated from the LC-tank (and the rest of the oscillator circuit).

The series-connected capacitor-resistor model (in Figure 2.4) reflects losses due to the relatively-low carrier doping level inside both $\mathrm{n}$-well and $\mathrm{p}$-substrate regions. Thus these resistors contribute to the quality factor degradation of the varactor structure.

The operating principle of this type of varactor is based on the capacitance across a p-n junction; and the relationship between the junction capacitance and applied reverse bias voltage is covered in the Grey\&Meyer's textbook [18]. A few related formulas are summarized as follows:

$$
\begin{gathered}
C_{j}=\frac{d Q}{d V_{R}}=\frac{d Q}{d W_{1}} \cdot \frac{d W_{1}}{d V_{R}} \\
d Q=A \cdot q \cdot N_{A} \cdot d W_{1} \\
\frac{d W_{1}}{d V_{R}}=\sqrt{\frac{\varepsilon}{2 \cdot q \cdot N_{A} \cdot\left(1+\frac{N_{A}}{N_{D}}\right) \cdot\left(\Psi_{0}+V_{R}\right)}}
\end{gathered}
$$


where

" $C_{j}$ " is the amount of capacitance across the $\mathrm{p}-\mathrm{n}$ junction

" $d Q$ " is the (incremental) amount of charge across the p-n junction

" $d W_{l}$ " is the (incremental) depth of the depletion region across the $\mathrm{p}-\mathrm{n}$ junction

" $A$ " is the cross-section area of the p-n junction

" $q$ " is the smallest unit of charge

" $N_{A}$ " is the doping density of acceptor atoms inside the p-type material

" $N_{D}$ " is the doping density of acceptor atoms inside the n-type material

" $V_{R}$ " is the applied reverse-bias voltage across the $\mathrm{p}-\mathrm{n}$ junction

" $\varepsilon$ " is the permittivity of silicon (with constant value of $1.04 \times 10^{-12} \mathrm{Farad} / \mathrm{cm}$ )

" $\Psi_{0} "$ is the built-in potential across the p-n junction

Substituting Equations 2.34 and 2.35 back to Equation 2.33:

$$
C_{j}=\frac{A \cdot \sqrt{\frac{q \cdot \varepsilon \cdot N_{A} \cdot N_{D}}{2 \cdot\left(N_{A}+N_{D}\right)}}}{\sqrt{\Psi_{0}+V_{R}}}=\frac{C_{j 0}}{\sqrt{1+\frac{V_{R}}{\psi_{0}}}}
$$

where 


$$
C_{j 0}=\lim _{(V R=0)} C_{j}=\frac{A \cdot \sqrt{\frac{q \cdot \varepsilon \cdot N_{A} \cdot N_{D}}{2 \cdot\left(N_{A}+N_{D}\right)}}}{\sqrt{\psi_{0}}}
$$

is the capacitance across the $\mathrm{p}-\mathrm{n}$ junction without any external bias voltage.

From Equation 2.36, capacitance across the pn-junction increases inversely with respect to the applied reverse-bias voltage; which also decreases the output frequency of the passive LC-VCO circuit. In other words, the output frequency of the oscillator circuit can be electrically tuned with varactors.

Each positive and negative output nodes of the oscillator circuit is connected to a group of 4-shunt-connected lateral PNP bipolar transistors. The "base" terminals of these 2 groups of lateral PNP devices are connected to a DC supply for frequency tuning purpose. Such lateral PNP series-shunt connection configuration is used to avoid the rectification effect across the emitter-base region, as voltage difference across this region varies from positive to negative (and vice versa) for every half of oscillation period. In other words, 4 of these 8 devices will have their emitter-base regions forward biased; and the other 4 will have their emitter-base regions reverse-biased at the same time instant. 
With a $2.02 \mathrm{nH}$ on-chip differential spiral inductor, the relationship between the single-ended tuning voltage and the LC-VCO circuit's output frequencies is summarized in the following table. Both capacitances and oscillator circuit output frequencies are verified with HSPICE simulations (on the schematic based netlist).

TABLE 2.2. Frequency tuning with 8 shunt connected PNP varactors

\begin{tabular}{lllll}
\hline Vtune & Cap_sim & Cap_sim_halved & Inductor & Frequency_sim \\
0.1 & $1.1 \mathrm{pF}$ & $0.55 \mathrm{pF}$ & $2.02 \mathrm{nH}$ & $4.788 \mathrm{GHz}$ \\
0.2 & $1.069 \mathrm{pF}$ & $0.5345 \mathrm{pF}$ & $2.02 \mathrm{nH}$ & $4.866 \mathrm{GHz}$ \\
0.3 & $1.031 \mathrm{pF}$ & $0.5155 \mathrm{pF}$ & $2.02 \mathrm{nH}$ & $4.923 \mathrm{GHz}$ \\
0.4 & $0.965 \mathrm{pF}$ & $0.4825 \mathrm{pF}$ & $2.02 \mathrm{nH}$ & $4.978 \mathrm{GHz}$ \\
0.5 & $0.995 \mathrm{pF}$ & $0.4975 \mathrm{pF}$ & $2.02 \mathrm{nH}$ & $5.040 \mathrm{GHz}$ \\
0.6 & $0.967 \mathrm{pF}$ & $0.4835 \mathrm{pF}$ & $2.02 \mathrm{nH}$ & $5.098 \mathrm{GHz}$ \\
0.7 & $0.937 \mathrm{pF}$ & $0.4685 \mathrm{pF}$ & $2.02 \mathrm{nH}$ & $5.1565 \mathrm{GHz}$ \\
0.8 & $0.9144 \mathrm{pF}$ & $0.4572 \mathrm{pF}$ & $2.02 \mathrm{nH}$ & $5.209 \mathrm{GHz}$ \\
0.9 & $0.9 \mathrm{pF}$ & $0.45 \mathrm{pF}$ & $2.02 \mathrm{nH}$ & $5.26 \mathrm{GHz}$ \\
1.0 & $0.88 \mathrm{pF}$ & $0.44 \mathrm{pF}$ & $2.02 \mathrm{nH}$ & $5.307 \mathrm{GHz}$ \\
1.1 & $0.8569 \mathrm{pF}$ & $0.42845 \mathrm{pF}$ & $2.02 \mathrm{nH}$ & $5.3515 \mathrm{GHz}$ \\
1.2 & $0.8487 \mathrm{pF}$ & $0.42435 \mathrm{pF}$ & $2.02 \mathrm{nH}$ & $5.3915 \mathrm{GHz}$ \\
1.3 & $0.8435 \mathrm{pF}$ & $0.42175 \mathrm{pF}$ & $2.02 \mathrm{nH}$ & $5.427 \mathrm{GHz}$ \\
1.4 & $0.837 \mathrm{pF}$ & $0.4185 \mathrm{pF}$ & $2.02 \mathrm{nH}$ & $5.461 \mathrm{GHz}$ \\
1.5 & $0.827 \mathrm{pF}$ & $0.4135 \mathrm{pF}$ & $2.02 \mathrm{nH}$ & $5.491 \mathrm{GHz}$ \\
1.6 & $0.8232 \mathrm{pF}$ & $0.4116 \mathrm{pF}$ & $2.02 \mathrm{nH}$ & $5.517 \mathrm{GHz}$ \\
1.7 & $0.7896 \mathrm{pF}$ & $0.3948 \mathrm{pF}$ & $2.02 \mathrm{nH}$ & $5.544 \mathrm{GHz}$ \\
1.8 & $0.7947 \mathrm{pF}$ & $0.39735 \mathrm{pF}$ & $2.02 \mathrm{nH}$ & $5.5675 \mathrm{GHz}$ \\
& & & &
\end{tabular}




\subsection{Active Components Of The "Tuned" Oscillator Circuit}

The active components of the implemented passive LC-VCO consist of two parts: a gain stage for the oscillator core circuit; and a multi-stage output buffer circuit. The gain stage compensates for the passive components' losses inside the oscillator core circuit (as described earlier), and sustains the oscillation signal amplitude. The output buffer circuit amplifies and delivers the oscillation signal from the oscillator core circuit to the off-chip PCB and test equipment. Details of the implemented gain stage and buffer stage circuits are covered in the following sections.

\subsubsection{Complementary Negative-gm Gain Stage}

A complementary topology is chosen for the implemented oscillator negative-gm gain stage, which consists of two shunt-connected PMOS and NMOS negative-gm circuits (see Figure 2.5). Each negative-gm circuit is made up of a pair of cross-coupled MOS devices; which are connected in a positive feedback configuration. For the purpose of achieving high oscillation frequecies, minimum gate lengths of $180 \mathrm{~nm}$ are chosen for all 
transistors in both PMOS and NMOS negative-gm gain stages. In order to improve rejection of interference caused by the power supply "vdd", a NMOS-based current mirror is used to provide the bias current of this negative-gm gain stage. As long as the NMOS current mirror circuit operates in saturation mode, which has been verified with transient simulations, the oscillator circuit operates in a mode commonly known as "current limit regime". In this mode, amplitude of the oscillator waveform is determined by both the bias current and the device sizes inside the complementary negative-gm gain stage; and does not swing from power supply to ground (i.e. rail to rail).

PMOS and NMOS devices are sized as follows...

(PMOS negative-gm gain stage) $\quad \mathrm{W} / \mathrm{L} / \mathrm{m}=2.5 \mathrm{um} / 0.18 \mathrm{um} / 16 * 2$

(NMOS negative-gm gain stage) $\quad \mathrm{W} / \mathrm{L} / \mathrm{m}=2.5 \mathrm{um} / 0.18 \mathrm{um} / 16^{*} 2$

(NMOS input current mirror) $\quad \mathrm{W} / \mathrm{L} / \mathrm{m}=5 \mathrm{um} / 0.4 \mathrm{um} / 4$

(NMOS output current mirror) $\quad \mathrm{W} / \mathrm{L} / \mathrm{m}=5 \mathrm{um} / 0.4 \mathrm{um} / 20$ 


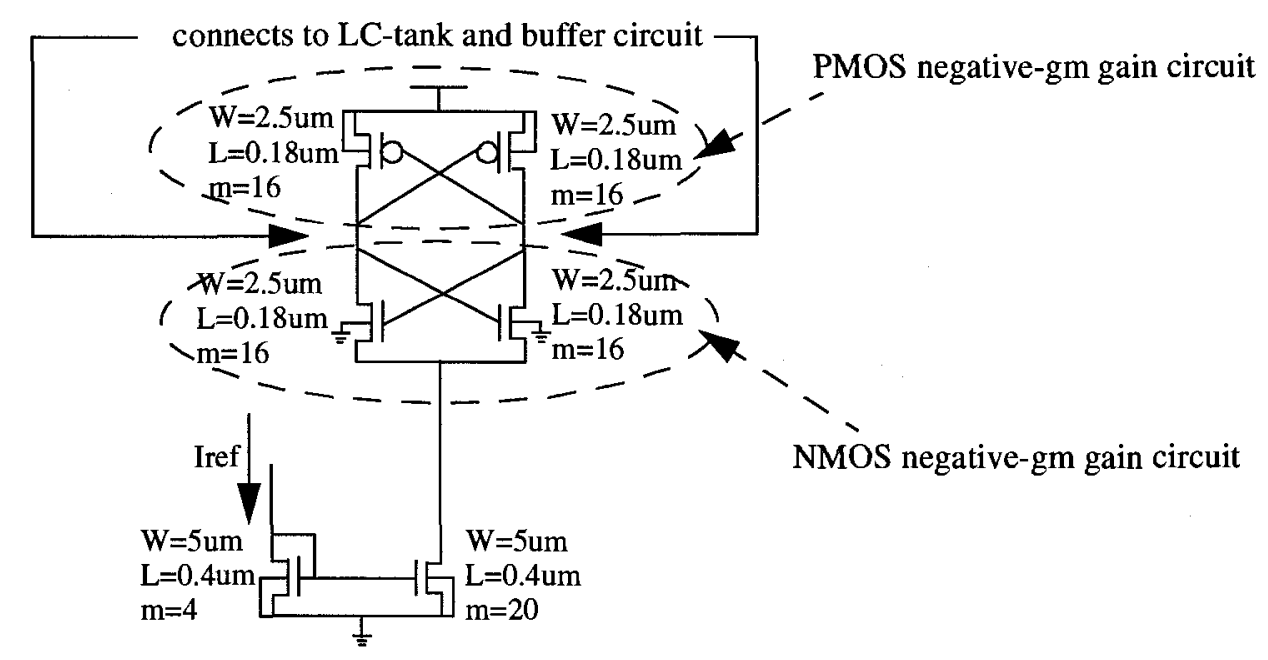

Figure 2.5: Schematic of complementary negative-gm gain stage (with MOS sizes)

\subsubsection{Passive LC-VCO Buffer Stage}

The objective of the output buffer circuit is to isolate the capacitive/inductive loading of the off-chip environment from the on-chip oscillator cirucit. The buffer circuit is composed of two inverting "resistively-loaded-differential-pair" gain stages, followed by a non-inverting "common-drain" output stage. Hence the polarity of the oscillation signals is preserved. 
The design methodology of the buffer stages is similar to CMOS inverter signalling, such that drive strength increases progressively from the core circuit to the off-chip environment. The only difference between CMOS signalling and current-steering type of signalling is in the current consumption of the gain stages. For the CMOS signalling approach, current consumption is controlled by the sizing of the devices and the power supply; whereas the current consumption in the current-steering type is controlled by the bias tail current of each gain stage. The device dimensions are summarized as follows:

Reference: (NMOS input current mirror, bias)W/L/m=5um/0.4um/4

1st stage: (NMOS output current mirror, bias)W/L/m=5um/0.4um/4

1st stage: (NMOS differential pair, each device)W/L/m=1.5um/0.18um/4

1st stage: $(\operatorname{Rload}=811.304+405.652) \quad \mathrm{W} / \mathrm{L} / \mathrm{m}=0.92 \mathrm{um} / 2.4 \mathrm{um} / 1+(1 / / 1)$

2nd stage: (NMOS output current mirror, bias) $\mathrm{W} / \mathrm{L} / \mathrm{m}=5 \mathrm{um} / 0.4 \mathrm{um} / 8$

2nd stage: (NMOS differential pair, each device)W/L/m=1.5um/0.18um/8

2nd stage: $($ Rload=405.652) W/L/m=0.92um/2.4um/(1//1)

3rd stage: (NMOS output current mirror, bias)W/L/m=5um/0.4um/12

3rd stage: (NMOS source follower) $\quad \mathrm{W} / \mathrm{L} / \mathrm{m}=2.5 \mathrm{um} / 0.18 \mathrm{um} / 32$ 


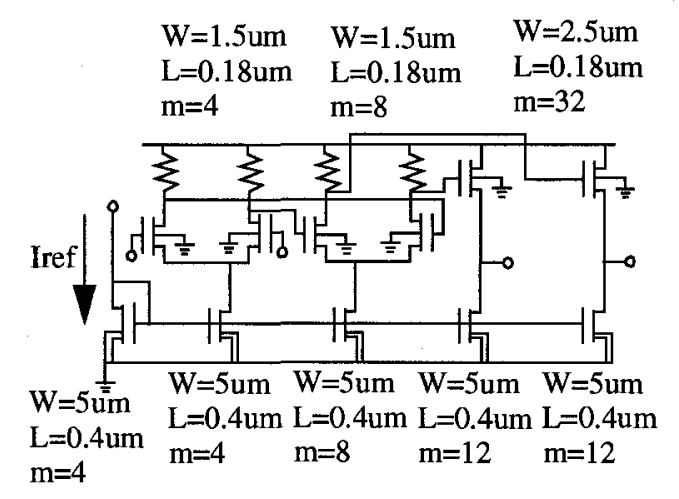

Figure 2.6: Schematic of passive LC-VCO buffer circuit (with MOS sizes)

In addition to the progressive increase in sizing of bias current of each buffer stage, the resistive load values of the two differential pair circuits are also scaled inversely with the bias current of the corresponding stage. Thus the amplitude of the output signal can be maintained relatively constant throughout the buffer circuit.

\subsection{Passive LC-VCO circuit operation mechanism}

This section covers the basic oscillator circuit operation mechanism. Illustration and description of a complementary negative-gm LC-VCO circuit topology will be cov- 
ered in the first sub-section, and the operation mechanism of this circuit will be covered in the second sub-section.

\subsubsection{Complementary LC-VCO circuit topology}

A complementary negative-gm LC-VCO circuit can be re-arranged as a 2-stage tuned amplifier connected in a positive feedback configuration (see Figure 2.7). Each tuned amplifier stage employs a CMOS inverter as an amplifying element, and the load is a narrow band LC-tank circuit. The NMOS tail current device provides bias current to the two CMOS inverter circuits. 


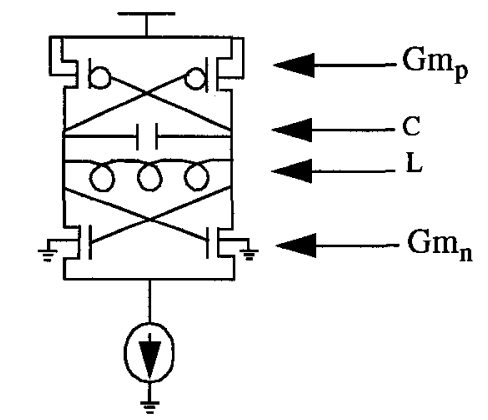

(complementary negative-gm LC-VCO)

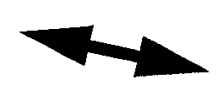

(2-stage tuned amplifier in +ve feedback config)

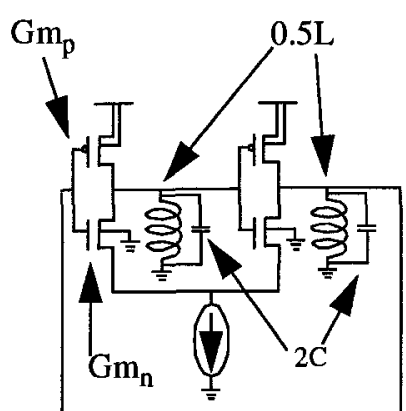

Figure 2.7: Similarity between a complementary negative-gm LC-VCO and a 2-stage tuned amplifier (in positive feedback configuration)

Since both series-connected-inductors and shunt-connected-capacitors are linearly added together, a single-ended version of the LC-tank passive components are shown as $0.5 \mathrm{~L}$ and $2 \mathrm{C}$ in the 2 -stage tuned amplifier circuit.

\subsubsection{Complementary negative-gm LC.VCO operation mechanism}

In the following illustration, each stage of a tuned amplifier drives a shunt-connected L-R-C load. The current bias NMOS device is replaced with the ideal ground (i.e. 
NMOS device source nodes are grounded). The small signal transfer function is summarized as follows:

$$
V_{o 1}(s)=-g_{m 1} \cdot V_{i 1} \cdot \frac{1}{Y_{\tan k}}=-g_{m 1} \cdot \frac{V_{i 1}(s)}{\left(\frac{1}{R_{p}}+\frac{1}{s L_{p}}+s C_{p}\right)} \rightarrow \frac{V_{o 1}(s)}{V_{i 1}(s)}=\frac{-g_{m 1}}{\left(\frac{1}{R_{p}}+\frac{1}{s L_{p}}+s C_{p}\right)}
$$

where $\mathrm{R}_{\mathrm{p}}$ incorporates all parasitic resistance from on-chip inductor, varactors, pmos and nmos devices; and each small signal transconductance (i.e. $g_{m 1}$ and $g_{m 2}$ ) includes contributions from both PMOS and NMOS devices.

Thus for a 2-stage tuned amplifier composed of identical shunt-connected L-R-C loads, the small signal loop gain will be:

$$
G(s)=\frac{V_{o 2}(s)}{V_{i 2}(s)} \cdot \frac{V_{o 1}(s)}{V_{i 1}(s)}=\frac{g_{m 1} \cdot g_{m 2}}{\left(\frac{1}{R_{p}}+\frac{1}{s L_{p}}+s C_{p}\right)^{2}}
$$

The impedance of the shunt-connected L-R-C tank circuit can be expressed as:

$$
Z_{\tan k}=\frac{1}{Y_{\tan k}}=\frac{1}{\left(\frac{1}{R_{p}}+\frac{1}{s L_{p}}+s C_{p}\right)}=\frac{1}{\frac{1}{R_{p}}+j\left(\omega C_{p}-\frac{1}{\omega L_{p}}\right)}=\frac{\frac{1}{R_{p}}-j\left(\omega C_{p}-\frac{1}{\omega L_{p}}\right)}{\left(\frac{1}{R_{p}}\right)^{2}+\left(\omega C_{p}-\frac{1}{\omega L_{p}}\right)^{2}}
$$


and

$$
\left[\frac{1}{\left(\frac{1}{R_{p}}+\frac{1}{s L_{p}}+s C_{p}\right)}\right]^{2}=\frac{1}{\left[\left(\frac{1}{R_{p}}\right)^{2}+\left(\omega C_{p}-\frac{1}{\omega L_{p}}\right)^{2}\right]^{2}} \cdot\left[\left(\frac{1}{R_{p}}\right)^{2}-\left(\omega C_{p}-\frac{1}{\omega L_{p}}\right)^{2}-\left(\frac{j 2}{R_{p}}\right) \cdot\left(\omega C_{p}-\frac{1}{\omega L_{p}}\right)\right]
$$

Substitute Equation 2.41 back into Equation 2.39, the small signal loop gain transfer function can be re-written as:

$$
G(s=j \omega)=\frac{g_{m 1} \cdot g_{m 2}}{\left[\left(\frac{1}{R_{p}}\right)^{2}+\left(\omega C_{p}-\frac{1}{\omega L_{p}}\right)^{2}\right]^{2}} \cdot\left[\left(\frac{1}{R_{p}}\right)^{2}-\left(\omega C_{p}-\frac{1}{\omega L_{p}}\right)^{2}-\left(\frac{j 2}{R_{p}}\right) \cdot\left(\omega C_{p}-\frac{1}{\omega L_{p}}\right)\right]
$$

This small signal loop gain transfer function is used to describe the inital condition for the positive feedback loop to start oscillation. As a rule of thumb, the magnitude of $\mathrm{G}(\mathrm{s})$ is chosen to be 3 or higher; in order to ensure proper oscillation during startup time.

For steady state operation, values of $\mathrm{C}_{\mathrm{p}}, \mathrm{L}_{\mathrm{p}}$, and $\mathrm{L}_{\mathrm{p}}$ are assumed to remain unchanged; whereas small signal transconductance $g_{m 1}$ and $g_{m 2}$ are now replaced with their equivalent large signal approximations $G_{m 1}$ and $G_{m 2}$. Equation 2.42 is now modified as: 


$$
G(s=j \omega)=\frac{G_{m 1} \cdot G_{m 2}}{\left[\left(\frac{1}{R_{p}}\right)^{2}+\left(\omega C_{p}-\frac{1}{\omega L_{p}}\right)^{2}\right]^{2}} \cdot\left[\left(\frac{1}{R_{p}}\right)^{2}-\left(\omega C_{p}-\frac{1}{\omega L_{p}}\right)^{2}-\left(\frac{j 2}{R_{p}}\right) \cdot\left(\omega C_{p}-\frac{1}{\omega L_{p}}\right)\right]
$$

The requirement for sustained oscillation from this two stage tuned amplifier circuit (connected in positive feedback configuration) is illustrated as follows:

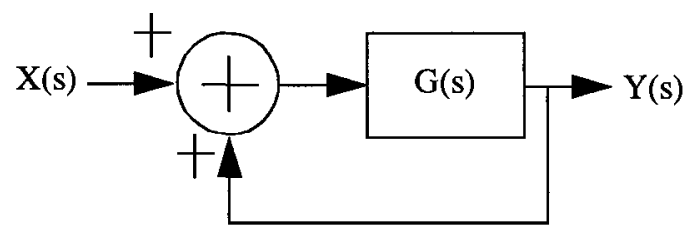

Figure 2.8: An illustration of a positive feedback loop with forward loop gain transfer function of $\mathbf{G}(\mathbf{s})$

From figure 2.8, the output signal of a closed loop system is:

$$
Y(s)=G(s) \cdot[X(s)+Y(s)] \rightarrow Y(s) \cdot[1-G(s)]=G(s) \cdot X(s)
$$

where $\mathrm{X}(\mathrm{s})$ is the input signal for the closed loop system.

The closed loop transfer function of a positive feedback loop is: 


$$
H(s)=\frac{Y(s)}{X(s)}=\frac{G(s)}{1-G(s)}
$$

For a closed loop system with sustained oscillation, the characteristic equation "1G(s)" must be set to zero. This is also governed by the "Barkhausen Criterion" in Sedra/ Smith's Microelectronic Circuits textbooks [25], and is summarized as follows:

$$
|G(s)|=1 \leftrightarrow \angle G(s)=0
$$

Similarly, the real and imaginary parts of the loop gain transfer function must be one and zero respectively. By substituting this new critera, Equation 2.43 can be re-written as:

$$
\angle G(s)=0 \rightarrow \operatorname{Im}\{G(s)\}=0 \rightarrow \frac{j 2}{R_{p}} \cdot\left(\omega_{o} C_{p}-\frac{1}{\omega L_{p}}\right)=0 \rightarrow\left(\omega_{o} C_{p}-\frac{1}{\omega_{o} L_{p}}\right)=0 \rightarrow \omega_{o}=\frac{1}{\sqrt{L_{p} \cdot C_{p}}}
$$

which implies a steady state resonant frequency of:

$$
\omega_{o}=\frac{1}{\sqrt{L_{p} \cdot C_{p}}} \rightarrow\left[f_{o}=\frac{\omega_{o}}{2 \pi}=\frac{1}{2 \pi} \cdot \frac{1}{\sqrt{L_{p} \cdot C_{p}}}\right]
$$


By substituting Equation 2.47 back to Equation 2.43, the magitude of the loop gain transfer function at resonant frequency can be determined as:

$$
\left|G\left(s=j \omega_{o}\right)\right|=1 \rightarrow\left|\frac{G_{m 1} \cdot G_{m 2}}{\left[\left(\frac{1}{R_{p}}\right)^{2}+\left(\omega_{o} C_{p}-\frac{1}{\omega L_{p}}\right)^{2}\right]^{2}} \cdot\left[\left(\frac{1}{R_{p}}\right)^{2}-0^{2}-0\right]\right|=1 \rightarrow\left|G_{m 1} \cdot G_{m 2} \cdot R_{p}^{2}\right|=1
$$

If we assume the two stages of amplifiers and tuned loads are identical, then:

$$
\left|G_{m 1} \cdot G_{m 2} \cdot R_{p}^{2}\right|=1 \rightarrow\left|G_{m 1} \cdot R_{p}\right| \cdot\left|G_{m 2} \cdot R_{p}\right|=1 \rightarrow\left|G_{m}\right|=\left|G_{m 1}\right|=\left|G_{m 2}\right|=\left|\frac{1}{R_{p}}\right|
$$

For steady state oscillation, Equation 2.38 can be re-written as:

$$
\frac{V_{o 1}\left(s=j \omega_{o}\right)}{V_{i 1}\left(s=j \omega_{o}\right)}=\frac{V_{o 2}\left(s=j \omega_{o}\right)}{V_{i 2}\left(s=j \omega_{o}\right)}=\frac{-G_{m}}{\frac{1}{R_{p}}+j\left(\omega_{o} C_{p}-\frac{1}{\omega_{o} L_{p}}\right)}=-\left(G_{m} \cdot R_{p}\right)=(-1) \cdot \frac{1}{R_{p}} \cdot R_{p}=-1
$$

Hence each amplifier inside the positive feedback system must provide a transconductance of $-1 / R_{p}$, which cancels with the parasitic loss of the corresponding L-C load. Thus signal oscillation is sustained with loss compensation; and this type of oscillator is commonly named as "negative gm LC-VCO". 


\subsection{Passive LC-VCO noise sources}

This section covers the potential noise sources found in the proposed complementary LC-VCO circuit; namely the PMOS/NMOS differential latches, the bias tail current NMOS device, and the series resistance inside the on-chip spiral inductor (caused by ohmic losses in metal and substrate, see Figure 2.9). Diagrams and equations are reproduced from one of Hajimiri's papers published in May of 1999 [10].

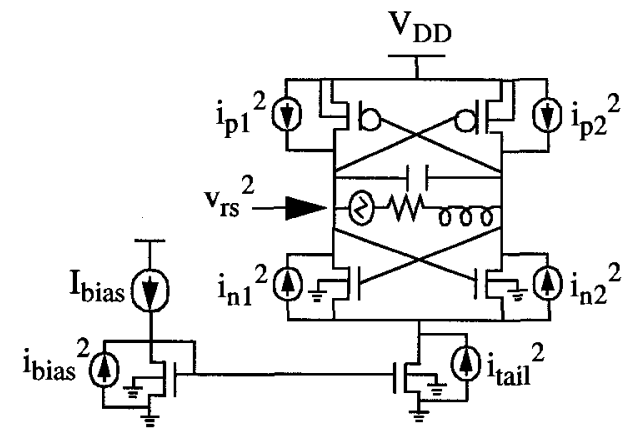

Figure 2.9: Complementary negative-gm LC-VCO with noise sources

The total differential noise power from the two NMOS and PMOS cross-coupled devices are summarized as follows:

$$
\overline{i_{\text {cross - coupled }}{ }^{2}}=\frac{1}{4} \cdot\left(\overline{i_{n 1}{ }^{2}}+\overline{i_{n 2}{ }^{2}}+\overline{i_{p 1}{ }^{2}}+\overline{i_{p 2}{ }^{2}}\right)=\frac{1}{2} \cdot\left(\overline{i_{n}{ }^{2}}+\overline{i_{p}{ }^{2}}\right)
$$


where

$$
\overline{i_{n 1}^{2}}=\overline{i_{n 2}{ }^{2}}=\overline{i_{n}^{2}}
$$

and

$$
\overline{i_{p 1}^{2}}=\overline{i_{p 2}^{2}}=\overline{i_{p}^{2}}
$$

are the equivalent current noise sources of each NMOS and PMOS cross-coupled latch device respectively.

The noise spectral densities of both NMOS and PMOS cross-coupled latch devices are:

$$
\overline{\overline{i_{n}}} \overline{\Delta f}=4 k T \gamma \mu_{n} C_{o x} \cdot\left(\frac{W_{n}}{L_{n}}\right) \cdot\left(V_{G S}-V_{T N}\right)
$$

and

$$
\overline{\overline{i_{p}}} \overline{\Delta f}=4 k T \gamma \mu_{p} C_{o x} \cdot\left(\frac{W_{p}}{L_{p}}\right) \cdot\left(V_{G S}-V_{T P}\right)
$$


where the value for " $\gamma$ ' is about $2 / 3$ for long-channel MOS devices; and between 2 and 3 for short-channel MOS devices. The increase in the " $\gamma$ " value is due to hot-electron effects inside the "short" channel.

In our proposed passive LC-VCO circuit, a 1:m NMOS current mirror supplies the needed current to both PMOS and NMOS cross-coupled latches. Hence the current noise power can be summarized as:

$$
\overline{i_{\text {tail }}{ }^{2}}=\overline{i_{\text {bias }}{ }^{2}} \cdot m^{2}
$$

which reveals that the choice of the bias current mirror ratio has a significant impact on the noise contribution.

Finally, the noise current density originated from the on-chip spiral inductor's "resistive loss" can be summarized as:

$$
\frac{\overline{i_{r s}^{2}}}{\Delta f}=4 k T \cdot \frac{r_{s} \cdot C}{L}=\frac{4 k T}{R_{p}}
$$

where 


$$
R_{p} \approx Q^{2} \cdot r_{s}=\frac{\left(L \cdot \omega_{0}\right)^{2}}{r_{s}}
$$

is the equivalent shunt resistance found at the LC-VCO oscillation frequency.

Device flicker and white noise sources with frequency contents close to harmonics of the oscillator circuit output frequency directly affects the oscillator circuit phase noise performance. The up/down-conversion mechanism of these noises onto oscillator output phase noise will be illustrated in the discussion section (i.e. Chapter 5) of this thesis.

\subsection{Passive LC-VCO Summary}

This chapter outlines the design of a passive inductor-capacitor voltage controlled oscillator circuit. A 2nH on-chip differential spiral inductor has been optimized with a software program called ASITIC. The frequency tuning element for this oscillator circuit, varactor, has been implemented by two groups of shunt-connected lateral PNP bipolar transistors. In the following chapter, the topic of impedance transformation and its application on oscillator circuit design will be covered. 


\section{CHAPTER 3 Active Inductor- Capacitor Voltage Controlled Oscillator}

This chapter covers the concept of active inductance generation, through the use of a gyrator and capacitor; and its application as a current controlled oscillator circuit. The first section provides an explanation and derivation of the active inductance concept. The second section gives a more in-depth look into active inductance generation; from a small signal perspective. The third section covers the implementation of a 2 -stage differential voltage controlled oscillator design, which is based on the active inductance generation concept. The fourth section reveals the frequency tuning mechanism of the implemented oscillator circuit, and its similarities with other circuits (i.e. ring oscillator and frequency divider). 


\subsection{Active Inductance Generation}

The concept of active inductance generation from gyrator-capacitor feedback loop orginates back in 1960's [2], and the most attractive feature of this circuit is the capability of performing impedance transformation. Hence the replacement of a physical passive spiral inductor by an gyrator-capacitr feedback circuit would greatly reduce the overall circuit area. The passive compoent of a gyrator-capacitor feedback circuit is a capacitor, whereas the active components consist of a positive and a negative transconductor placed in a feedback loop (see Figure 3.1). 


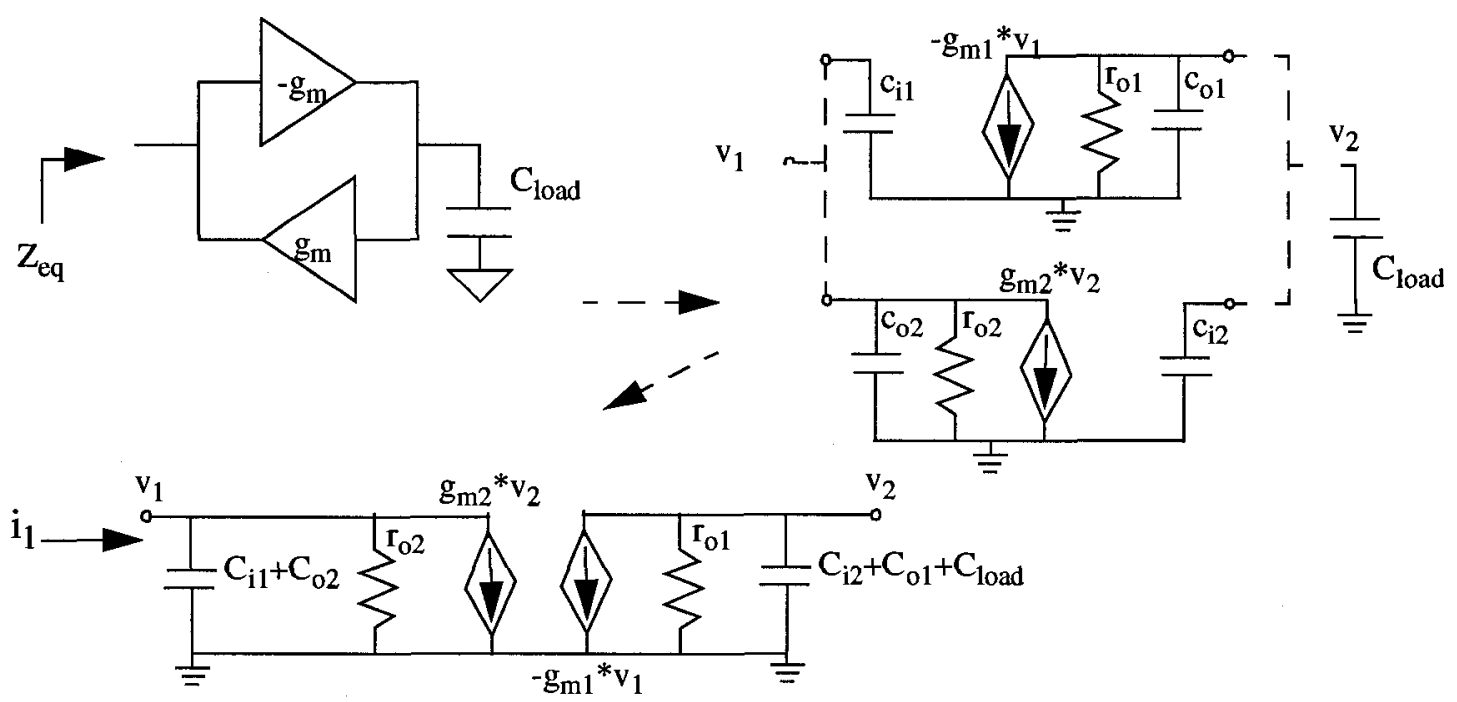

Figure 3.1: gyrator-capacitor circuit and its small signal models

The impedance transformation (i.e. from capacitive loading to inductive loading) can be derived with small signal models of the above circuit and Kirchhoff's Current Law as follows:

$\mathrm{KCL}$ at node $\mathrm{v}_{1}$ :

$$
i_{1}=\left[s \cdot\left(C_{i 1}+C_{o 2}\right)+\frac{1}{r_{o 2}}\right] \cdot v 1+\left(g_{m 2} \cdot v_{2}\right)
$$

where $i_{1}$ is defined as the small signal current going into node $v_{1}$. 
KVL inside loop between ground and node $v_{2}$ :

$$
v_{2}=\left(g_{m 1} \cdot v_{1}\right) \cdot\left[\frac{1}{\frac{1}{r_{o 1}}+s \cdot\left(C_{o 1}+C_{i 2}+C_{l o a d}\right)}\right]
$$

Substitute Equation 3.2 into the variable $\mathrm{v}_{2}$ of Equation 3.1:

$$
\frac{i_{1}}{v_{1}}=s \cdot\left(C_{i 1}+C_{o 2}\right)+g_{o 2}+\frac{g_{m 1} \cdot g_{m 2}}{g_{o 1}+s \cdot\left(C_{o 1}+C_{i 2}+C_{l o a d}\right)}
$$

where $g_{o 1}=1 / r_{o 1}$ and $g_{o 2}=1 / r_{o 2}$.

After dividing the product " $\mathrm{g}_{\mathrm{m} 1} * \mathrm{~g}_{\mathrm{m} 2}$ " from both numerator and denominator of the third component on the right hand side, the equivalent admittance of this circuit becomes:

$$
\frac{1}{Z_{e q}}=\frac{i_{1}}{v_{1}}=g_{o 2}+s \cdot\left(C_{i 1}+C_{o 2}\right)+\frac{1}{\frac{g_{o 1}}{g_{m 1} \cdot g_{m 2}}+\frac{s \cdot\left(C_{o 1}+C_{i 2}+C_{l o a d}\right)}{g_{m 1} \cdot g_{m 2}}}
$$

From the above circuit admittance equation, the three components on the right hand side represent the result of the impedance transformation. The first component " $\mathrm{g}_{\mathrm{o} 2}$ " 
is the admittance (or inverse of the impedance) between node $v_{1}$ and ground (or GND).

The second component " $\mathrm{s} *\left(\mathrm{C}_{\mathrm{i} 1}+\mathrm{C}_{\mathrm{o} 2}\right)$ " is the sum of parasitic capacitors $\mathrm{C}_{\mathrm{i} 1}$ and $\mathrm{C}_{\mathrm{o} 2}$ seen between node $\mathrm{v}_{1}$ and ground. The third component is the admittance of an inductor with series resistance. The first fraction term in the denominator of the third component is the series resistance, as it does not have any frequency dependent component (i.e. $s=j \omega)$ ).

The second fraction term in the denominator of the third component is the impedance of an inductor, with a value of " $\mathrm{s} *\left(\mathrm{C}_{\mathrm{o} 1}+\mathrm{C}_{\mathrm{i} 2}+\mathrm{C}_{\mathrm{load}}\right) /\left(\mathrm{g}_{\mathrm{m} 1} * \mathrm{~g}_{\mathrm{m} 2}\right)$ "; the "s" term indicates its positive linear dependency on frequency. Note that this term results from an impedance transformation via the feedback loop, namely from both explicit and parasitic capacitive loading (at node v2) transformed into an inductive behaviour (when observed from node v1).

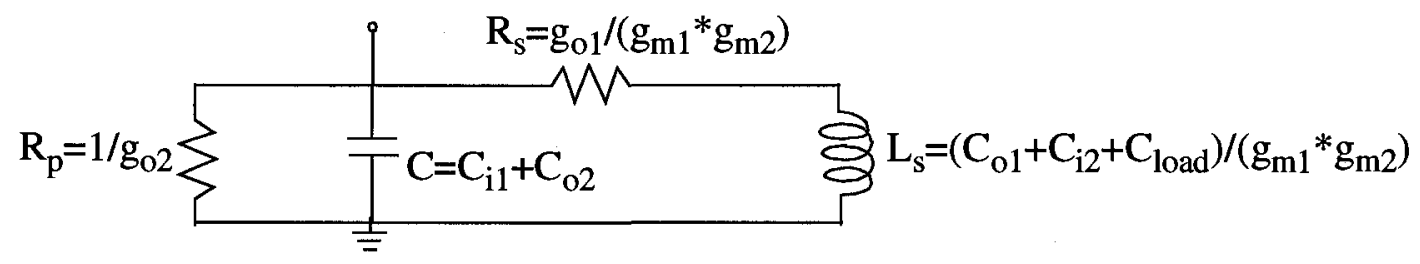

Figure 3.2: tank circuit resulted from active inductance circuit 
Upon comparison with a shunt-connected inductor-capacitor (or simply LC-tank) circuit, the resistances " $\mathrm{R}_{\mathrm{p}}=\mathrm{g}_{02}$ " and " $\mathrm{R}_{\mathrm{s}}=\mathrm{g}_{01} /\left(\mathrm{g}_{\mathrm{m} 1}{ }^{*} \mathrm{~g}_{\mathrm{m} 2}\right)$ " are components that reduce the quality factor ( $Q$ factor) of such a tank circuit. For an ideal LC-tank circuit (i.e. quality factor of infinity), values of " $R_{p}$ " and " $R_{s}$ " should be infinity and zero respectively; which requires the values of both " $\mathrm{g}_{01}$ " and " $\mathrm{g}_{02}$ " to be zero. Finally, both " $\mathrm{R}_{\mathrm{s}}$ " and " $\mathrm{L}_{\mathrm{s}}$ " terms vary inversely with the product term " $\mathrm{g}_{\mathrm{m} 1} * \mathrm{~g}_{\mathrm{m} 2}$ "; which provides an opportunity of tuning, as values of " $\mathrm{g}_{\mathrm{m} 1}$ " and " $\mathrm{g}_{\mathrm{m} 2}$ " can be adjusted by varying the bias current of transconductive elements " $\mathrm{g}_{\mathrm{m}}$ " and " $\mathrm{g}_{\mathrm{m}}$ ". This leads to our research focus on tunable active inductance generation and its application as a current controlled oscillator circuit. The details on oscillation frequency tuning mechanism will be covered in later sections of this chapter.

\subsection{Implementation Of Active Inductance Generation}

From Section 3.1, active elements " $\mathrm{g}_{\mathrm{m} 1}$ " and " $\mathrm{g}_{\mathrm{m} 2}$ " in the gyrator-capacitor feedback loop circuit are generalized as transconductance components. In fact, each of these stages can be as complicated as a multi-stage transconductance amplifier; or as simple as a 
single transistor device. The illustration in Figure 3.1 includes only input capacitance, output capacitance, output resistance, and voltage controlled current source as the complete model for such a transconductive component; which can easily be interpretted as a MOSFET device. For a complete device model, a capacitor will be added between the input capacitor and the output transconductive element (i.e. to represent the parasitic junction capacitor $\mathrm{C}_{\mathrm{gd}}$ in a MOS transistor, see Figure 3.3).
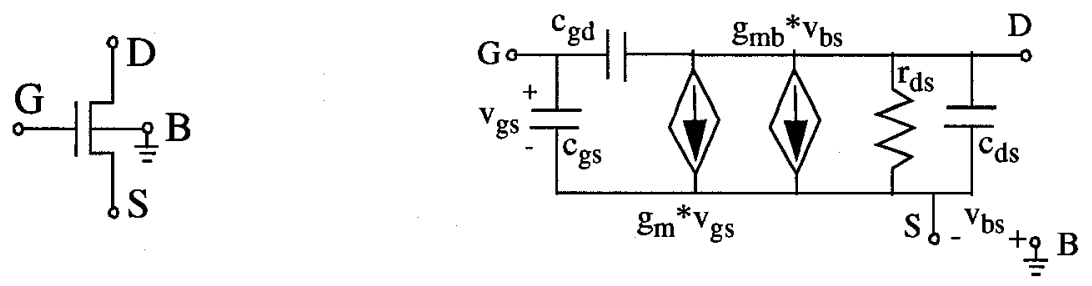

Figure 3.3: MOSFET (NMOS) device and its small signal model

Similarly, bipolar devices can be modelled with a similar diagram by adding two extra components (see Figure 3.4): an input resistance in parallel with the input capacitance (i.e. to represent the $r_{\pi}$ component in a bipolar transistor), and a capacitor between the input capacitor and the output transconductive element (i.e. to represent the feedback junction capacitor $\mathrm{C}_{\mu}$ in a bipolar transistor). By connecting in the same gyrator-capacitor feedback loop manner, the terms " $\mathrm{r}_{\mathrm{o} 1}$ " and " $\mathrm{r}_{\mathrm{o} 2}$ " can be simply modified as " $\mathrm{r}_{\mathrm{o} 1}{ }^{*} \mathrm{r}_{\mathrm{i} 2}$ ' 
$\left(\mathrm{r}_{\mathrm{o} 1}+\mathrm{r}_{\mathrm{i} 2}\right)$ " and " $\mathrm{r}_{\mathrm{O} 2}{ }^{*} \mathrm{r}_{\mathrm{i} 1} /\left(\mathrm{r}_{\mathrm{o} 2}+\mathrm{r}_{\mathrm{i} 1}\right)$ " respectively. Thus input and output terminals of component " $\mathrm{g}_{\mathrm{m} 1}$ " are connected to output and input resistance of component " $\mathrm{g}_{\mathrm{m} 2}$ " respectively.
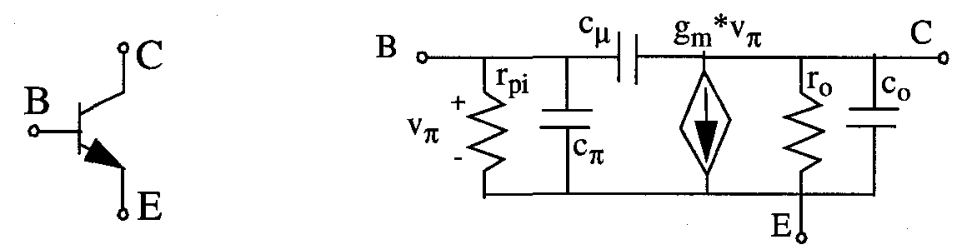

Figure 3.4: Bipolar (NPN) device and its small signal model

In this research, the gyrator-capacitor feedback loop circuit is implemented in TSMC 0.18um CMOS technology. Since a MOS device can be biased in either "common-drain", "common-gate", or "common-source" configuration, three combination of such configurations have been investigated for the gyrator-capacitor feedback loop topology: "common-drain, common-source", "common-gate, common-source", and "commonsource, common-source".

\subsection{1 “Common-drain, Common-source” feedback loop}

A "Common-drain" configuration is usually used as a voltage buffer, because the amplitude of input signals are mostly preserved. Input signals are level shifted by a DC 
voltage, which is determined by both the MOS device threshold voltage and the bias current of the "common-drain" stage.

In the gyrator-capacitor feedback loop topology, the "common-drain" stage output current (increases as gate voltage increases) charges up the gate terminal of the "commonsource" stage, whereas the "common-source" stage sinks current and decreases the gate terminal voltage of the "common drain" stage. Hence the "common-drain" and the "common-source" MOS devices act as positive and negative transconductive components in the feedback loop respectively. 


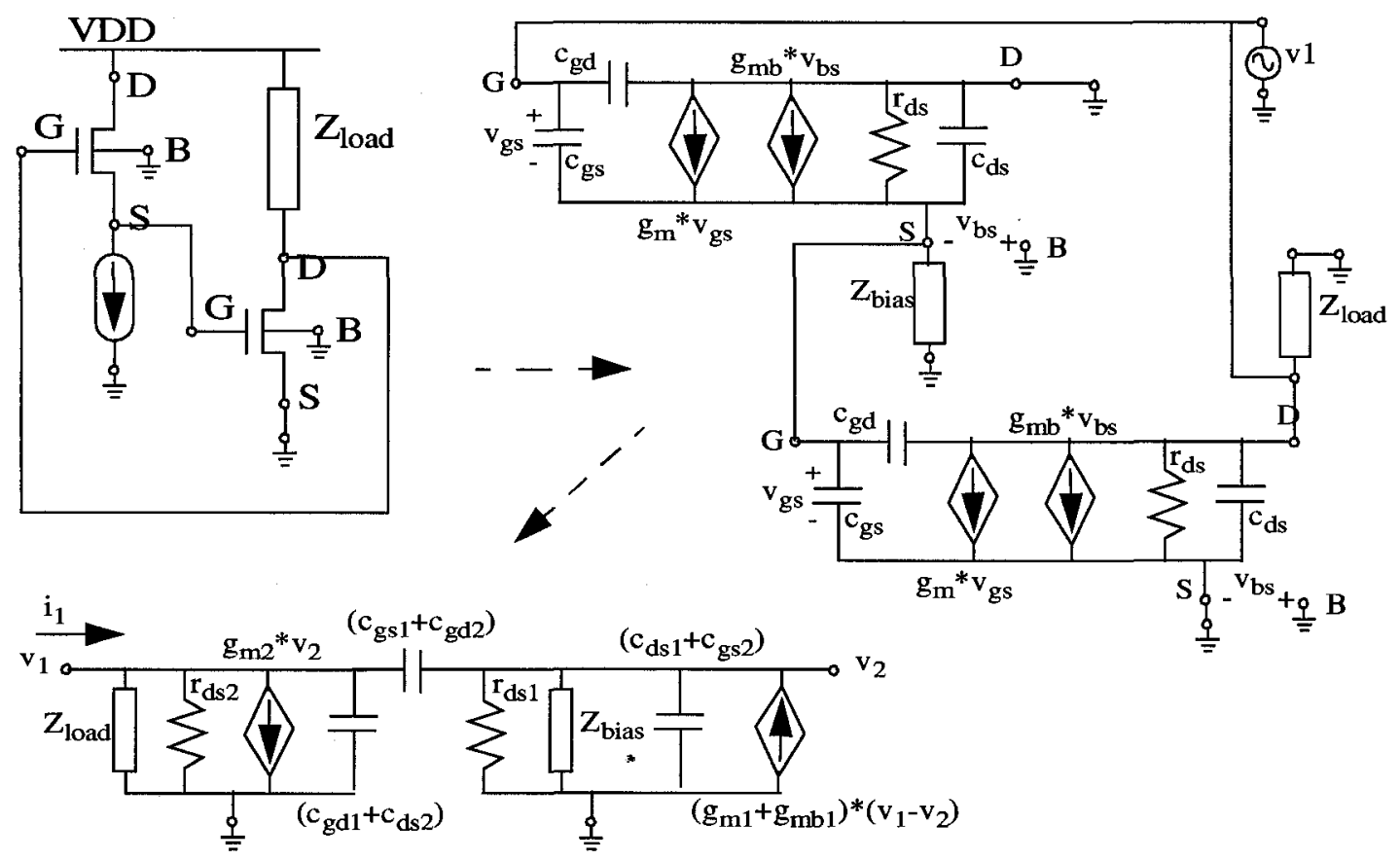

Figure 3.5: common-drain/common-source feedback configuration and its small signal models

The impedance transformation for this common-drain/common-source feedback circuit can be derived with small signal models of the above circuit and Kirchhoff's Current Law as follows:

KCL at node $\mathrm{v}_{1}$ : 


$$
i_{1}=\left[Y_{l o a d}+g_{d s 2}+s \cdot\left(C_{g d 1}+C_{g s 1}+C_{d s 2}+C_{g d 2}\right)\right] \cdot v_{1}+\left[g_{m 2}-s \cdot\left(C_{g s 1}+C_{g d 2}\right)\right] \cdot v_{2}
$$

where $i_{1}$ is defined as the current going into node $v_{1}, Y_{\text {load }}=1 / Z_{\text {load }}, g_{d s 2}=1 / r_{d s 2}$.

KCL at node $\mathrm{v}_{2}$ :

$$
\left(g_{m 1}+g_{m b 1}\right) \cdot\left(v_{1}-v_{2}\right)=\left[s \cdot\left(C_{g s 1}+C_{g d 2}\right)\right] \cdot\left(v_{2}-v_{1}\right)+\left[g_{d s 1}+Y_{b i a s}+s \cdot\left(C_{d s 1}+C_{g s 2}\right)\right] \cdot v_{2}
$$

where $\mathrm{Y}_{\mathrm{bias}}=1 / \mathrm{Z}_{\mathrm{bias}}$ and $\mathrm{g}_{\mathrm{ds} 1}=1 / \mathrm{r}_{\mathrm{ds} 1}$.

After re-arranging and isolating the $\mathrm{v}_{1}$ and $\mathrm{v}_{2}$ terms (of Equation 3.6):

$$
v_{2}=\frac{g_{m 1}+g_{m b 1}+s \cdot\left(C_{g s 1}+C_{g d 2}\right)}{g_{m 1}+g_{m b 1}+g_{d s 1}+Y_{b i a s}+s \cdot\left(C_{d s 1}+C_{g s 1}+C_{g d 2}+C_{g s 2}\right)} \cdot v_{1}
$$

Substitute Equation 3.7 into the variable $v_{2}$ of Equation 3.5, and re-arrange for $i_{1} /$ $\mathrm{v}_{1}$ :

$$
\frac{i_{1}}{v_{1}}=Y_{\text {parallel }}+s \cdot\left(C_{\text {parallel }}\right)+\frac{1}{Z_{\text {series }}+s \cdot\left(L_{\text {transformed }}\right)}
$$

where 


$$
\begin{gathered}
Y_{\text {parallel }}=Y_{\text {load }}+g_{d s 2} \\
C_{\text {parallel }}=C_{g d 1}+C_{g s 1}+C_{d s 2}+C_{g d 2} \\
Z_{\text {series }}=\frac{g_{m 1}+g_{m b 1}+g_{d s 1}+Y_{\text {bias }}}{g_{m 2} \cdot\left(g_{m 1}+g_{m b 1}\right)+s \cdot\left(C_{g s 1}+C_{g d 2}\right) \cdot\left(g_{m 2}-g_{m 1}-g_{m b 1}\right)-s^{2} \cdot\left(C_{g s 1}+C_{g d 2}\right)^{2}}
\end{gathered}
$$

and $\mathrm{L}_{\text {transformed }}$ represents the transformed inductance, as follows:

$$
L_{\text {transformed }}=\frac{C_{d s 1}+C_{g s 1}+C_{g d 2}+C_{g s 2}}{g_{m 2} \cdot\left(g_{m 1}+g_{m b 1}\right)+s \cdot\left(C_{g s 1}+C_{g d 2}\right) \cdot\left(g_{m 2}-g_{m 1}-g_{m b 1}\right)-s^{2} \cdot\left(C_{g s 1}+C_{g d 2}\right)^{2}}
$$

Similar to Equation 3.4 derived back in Section 3.1, Equations 3.8 to 3.12 represent the transformed admittance and impedance by the gyrator-capcitor feedback circuit (implemented with a common-drain stage, followed by a common-source stage). $\mathrm{Y}_{\text {parallel }}$ (of Equation 3.9) and $\mathrm{C}_{\text {parallel }}$ (of Equation 3.10) are the admittance and capacitance seen between the input node $v_{1}$ and ground respectively. $Z_{\text {series }}$ (of Equation 3.11) is the series resistance of the transformed inductance, whereas $L_{\text {transformed }}$ (of Equation 3.12) is the "inductance" generated by the gyrator-capacitor feedback circuit. 
Upon careful inspection of Equations 3.9, 3.10, 3.11, and 3.12, the first two equations are straight forward (i.e. similar format as Equation 3.4 in section 3.1); whereas there are extra terms in both numerator and denominator of Equations 3.11 and 3.12.

In the denominator polynominal of Equations 3.11 and 3.12, the " $\mathrm{s}$ " term becomes negligible, which is due to the product of our target oscillation frequency and the parasitic capacitance (i.e. range of giga-Hertz multiplied by femto-Farad, then squared). In addition, if both "common-drain" and "common-source" stages are biased to have similar $g_{m}$ values; then the $s$ term approaches zero (i.e. " $g_{m 2}-g_{m 1}$ "). Furthermore, " $g_{m b}$ " values are usually much smaller than the values of " $\mathrm{g}_{\mathrm{m}}$ ". Hence the donominator polynominal will be simplified to just the product of " $\mathrm{g}_{\mathrm{m} 1}$ " and " $\mathrm{g}_{\mathrm{m} 2}$ ", or simply " $\mathrm{g}_{\mathrm{m}}{ }^{2}$ ", which is identical to the denominator of both " $\mathrm{Z}_{\text {series }}$ " and " $\mathrm{L}_{\text {transformed" }}$ " terms in Equation 3.4 .

An alternate way to interpret the extra terms in the denominator polynomial (i.e.

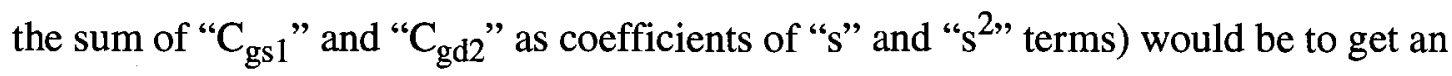
understanding on the effect of these capacitors, which form the feedforward (or bypass) path between the two transconductance stages. Hence for signals with frequency contents 
higher than the pole caused by these two capacitors, these signals would be "shorted" between the input and output nodes of the gyrator feedback loop; and the capacitance (or whatever load impedance) at node $\mathrm{v}_{2}$ would be seen by test voltage at node $\mathrm{v}_{1}$. In other words, the gyrator feedback loop ceases to perform the impedance transformation.

The sum of " $g_{d s 1}$ " and " $Y_{\text {bias }}$ " terms in the numerator of Equation 3.11 are due to their shunt connection between the loading node $\mathrm{v}_{2}$ and ground; whereas the " $\mathrm{g} \mathrm{m} 1$ " and " $\mathrm{g}_{\mathrm{mb} 1}$ " terms are due to the "common-drain" transconductance stage circuit topology. Since the "source" node of the MOS device is the output node, the signal at this node is not grounded; and a voltage exists across the tail current source (its admittance is denoted by the term "Y $\mathrm{Yias}$ "). Hence the current generated by this "common-drain" transconductance stage results from the product " $\left(\mathrm{g}_{\mathrm{m} 1}+\mathrm{g}_{\mathrm{mb} 1}\right) *\left(\mathrm{v}_{1}-\mathrm{v}_{2}\right)$ " instead of the simple term " $\mathrm{g}_{\mathrm{m} 1} * \mathrm{v}_{1}$ " derived back in Section 3.1. Note that the more admittance terms added to the numerator of this $\mathrm{Z}_{\text {series }}$ term, the bigger its value would be; which would further decrease the "Q-factor" of this active inductor (of value " $\mathrm{L}_{\text {transformed"). }}$

In the numerator of Equation 3.12, the sum of capacitors " $\mathrm{C}_{\mathrm{ds} 1}$ ", "C $\mathrm{C}_{\mathrm{gs} 1}$ ", " $\mathrm{C}_{\mathrm{gd} 2}$ ",

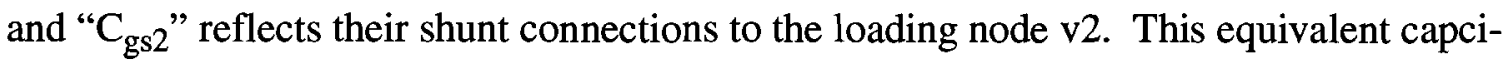


tor is then transformed to an "inductive" element by the "common-drain, common-source" gyrator feedback circuit; and the transformed inductor is represented by the term " $\mathrm{L}_{\text {trans- }}$ formed".

\subsection{2 “Common-gate, Common-source" feedback loop}

A "Common-gate" configuration is usually used as a current buffer, because the magnitude of the input signal current can be maintained whenever the MOS device operates in the "saturation" region.

From the transconductance gain perspective, the "common-gate" stage output current increases (with respect to our positive current convention as current going into a circuit node) as input voltage increases. This is due to the fact that the gate voltage is fixed at a DC bias level, and an increase at input (source node of MOS device) actually decreases the device current conduction; and causes a decrease of current taken away from the output node. The "common-source" stage sinks current (i.e. take current away from the output node) as its input voltage increases. Hence the "common gate" MOS device and the 
"common source" MOS device act as positive and negative transconductive components in the feedback loop respectively.

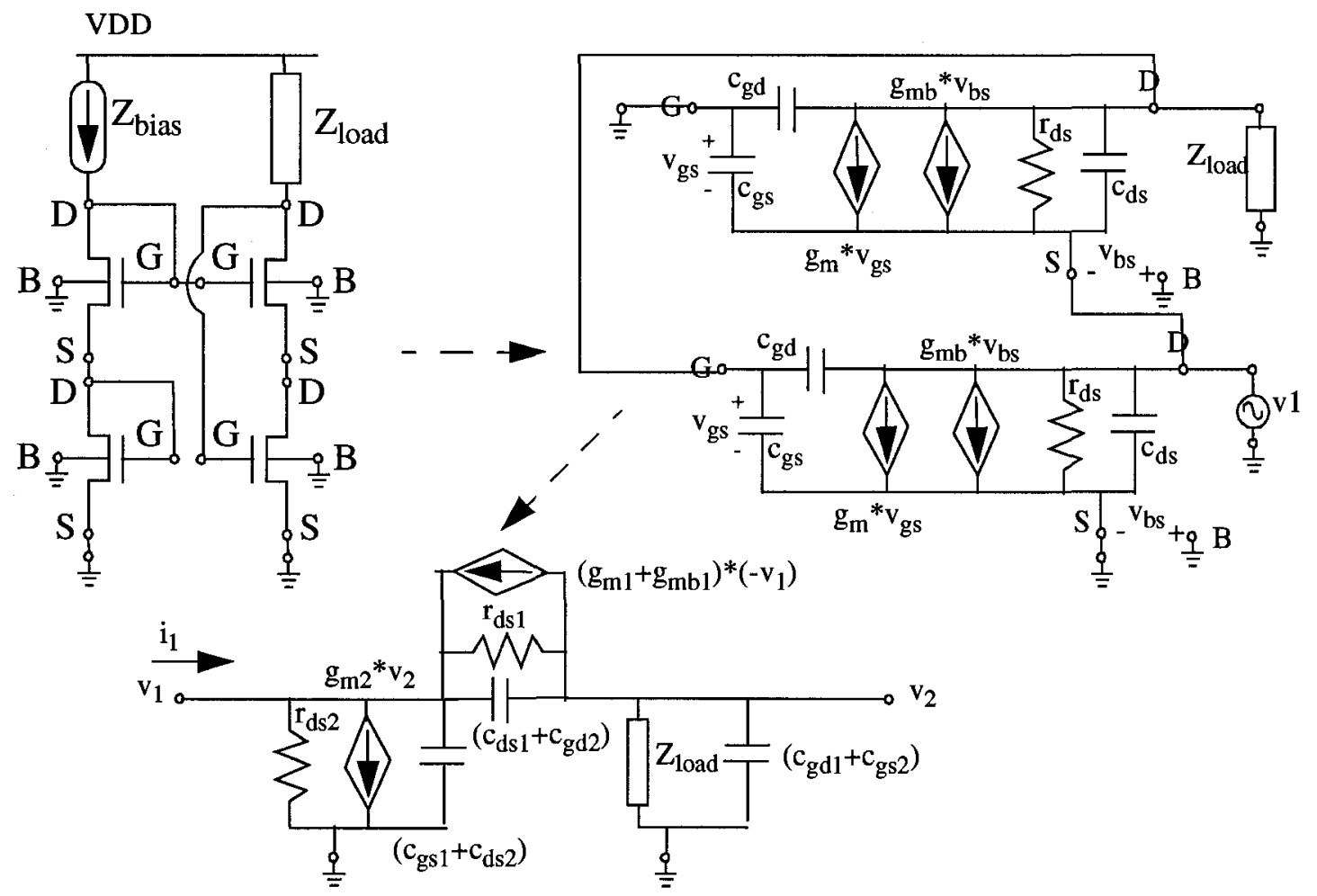

Figure 3.6: common-gate/common-source feedback configuration and its small signal models 
The impedance transformation for this common-gate/common-source feedback circuit can be derived with small signal models of above circuits and Kirchhoff's Current Law as follows:

$\mathrm{KCL}$ at node $\mathrm{v}_{1}$ :

$$
i_{1}=\left[g_{m 1}+g_{m b 1}+g_{d s 2}+s \cdot\left(C_{g s 1}+C_{d s 2}\right)\right] \cdot v_{1}+g_{m 2} \cdot v_{2}+\left[g_{d s 1}+s \cdot\left(C_{d s 1}+C_{g d 2}\right)\right] \cdot\left(v_{1}-v_{2}\right)
$$

where $\mathrm{i} 1$ is defined as the current going into node $v_{1}, g_{d s 1}=1 / r_{d s}, g_{d s}=1 / r_{d s 2}$.

After re-arranging and isolating the $\mathrm{v}_{1}$ and $\mathrm{v}_{2}$ terms in Equation 3.13:

$$
i_{1}=\left[Y_{11}\right] \cdot v_{1}+\left[Y_{12}\right] \cdot v_{2}
$$

where

$$
\begin{gathered}
Y_{11}=g_{m 1}+g_{m b 1}+g_{d s 1}+g_{d s 2}+s \cdot\left(C_{d s 1}+C_{g s 1}+C_{d s 2}+C_{g d 2}\right) \\
Y_{12}=g_{m 2}-g_{d s 1}-s \cdot\left(C_{d s 1}+C_{g d 2}\right)
\end{gathered}
$$

KCL at node $\mathrm{v}_{2}$ : 


$$
\left(g_{m 1}+g_{m b 1}\right) \cdot\left(-v_{1}\right)+\left[g_{d s 1}+s \cdot\left(C_{d s 1}+C_{g d 2}\right)\right] \cdot\left(v_{2}-v_{1}\right)+\left[Y_{l o a d}+s \cdot\left(C_{g d 1}+C_{g s 2}\right)\right] \cdot v_{2}=0
$$

where $\mathrm{Y}_{\text {load }}=1 / \mathrm{Z}_{\mathrm{load}}, \mathrm{g}_{\mathrm{ds} 1}=1 / \mathrm{r}_{\mathrm{ds} 1}$, and $\mathrm{g}_{\mathrm{ds} 2}=1 / \mathrm{r}_{\mathrm{ds} 2}$.

After re-arranging and isolating the $\mathrm{v}_{1}$ and $\mathrm{v}_{2}$ terms in Equation 3.17:

$$
v_{2}=\frac{g_{m 1}+g_{m b 1}+g_{d s 1}+s \cdot\left(C_{d s 1}+C_{g d 2}\right)}{Y_{l o a d}+g_{d s 2}+s \cdot\left(C_{d s 1}+C_{g d 1}+C_{g d 2}+C_{g s 2}\right)} \cdot v 1
$$

Substitute Equation 3.18 into the variable $v_{2}$ of Equation 3.14, and re-arrange for $\mathrm{i}_{1} / \mathrm{v}_{1}$ :

$$
\frac{i_{1}}{v_{1}}=Y_{\text {parallel }}+s \cdot\left(C_{\text {parallet }}\right)+\frac{1}{Z_{\text {series }}+s \cdot\left(L_{\text {transformed }}\right)}
$$

where

$$
\begin{gathered}
Y_{\text {parallel }}=g_{m 1}+g_{m b 1}+g_{d s 1}+g_{d s 2} \\
C_{\text {parallel }}=C_{d s 1}+C_{g s 1}+C_{d s 2}+C_{g d 2}
\end{gathered}
$$




$$
Z_{\text {series }}=\frac{Y_{l o a d}+g_{d s 2}}{d_{0}+s \cdot\left(C_{g s 1}+C_{g d 2}\right) \cdot\left(g_{m 2}-g_{m 1}-g_{m b 1}-2 \cdot g_{d s 1}\right)-s^{2} \cdot\left(C_{d s 1}+C_{g s 2}\right)^{2}}
$$

and $\mathrm{L}_{\text {transformed }}$ represents the transformed inductance:

$$
L_{\text {transformed }}=\frac{C_{d s 1}+C_{g d 1}+C_{g d 2}+C_{g s 2}}{d_{0}+s \cdot\left(C_{g s 1}+C_{g d 2}\right) \cdot\left(g_{m 2}-g_{m 1}-g_{m b 1}-2 \cdot g_{d s 1}\right)-s^{2} \cdot\left(C_{d s 1}+C_{g s 2}\right)^{2}}
$$

Once more, we have

$$
d_{0}=g_{m 2} \cdot\left(g_{m 1}+g_{m b 1}\right)+g_{d s 1} \cdot\left(g_{m 2}-g_{m 1}-g_{m b 1}\right)-\left(g_{d s 1}\right)^{2}
$$

Similar to the "common-drain, common-source" gyrator-capacitor feedback circuit, Equations 3.19 to 3.24 represent the transformed admittance and impedance by the gyrator-capcitor feedback circuit (implemented with a common-gate stage, followed by a common-source stage). $Y_{\text {parallel }}$ (of Equation 3.20) and $C_{\text {parallel }}$ (of Equation 3.21) are the admittance and capacitance seen between the input node $v_{1}$ and ground. $Z_{\text {series }}$ (of Equations 3.22 and 3.24 ) is the series resistance of the transformed inductance, whereas $\mathrm{L}_{\text {trans- }}$ formed (of Equations 3.23 and 3.24) is the transformed inductance generated by the gyrator-capacitor feedback circuit. 
In the denominator polynominal of Equations 3.22 and 3.23 , the $\mathrm{s}^{2}$ term becomes negligible, which is due to the product of our target oscillation frequency and the parasitic capacitance (i.e. range of giga-Hertz multiplied by femto-Farad, then squared). In addition, if both "common-gate" and "common-source" stages are biased to have similar $\mathrm{g}_{\mathrm{m}}$ values; then the $s$ term approaches to zero (i.e. $g_{m 2}-g_{m 1}$ ). Furthermore, $g_{m b}$ and $g_{d s}$ values are usually much smaller than the values of $g_{m}$. Hence the donominator polynominal will be simplified to $\mathrm{g}_{\mathrm{m} 1}{ }^{*} \mathrm{~g}_{\mathrm{m} 2}$, which is similar to the denominator of both $\mathrm{Z}_{\text {series }}$ and $\mathrm{L}_{\text {trans- }}$ formed terms in Equation 3.4.

An alternate way to interpret the extra terms in the denominator polynomial (i.e. " $\mathrm{g}_{\mathrm{m} 1}{ }^{*} \mathrm{~g}_{\mathrm{m} 2}-\mathrm{g}_{\mathrm{ds} 1}{ }^{2 ",}, " \mathrm{C}_{\mathrm{ds} 1}$ ", and " $\mathrm{C}_{\mathrm{gs} 2}$ " as coefficient of $\mathrm{s}^{0}, \mathrm{~s}$, and $\mathrm{s}^{2}$ terms) would be to get an understanding on the effect of these device-related parasitic components: " $\mathrm{g}_{\mathrm{ds} 1}$ " (or inverse of $\mathrm{r}_{\mathrm{ds} 1}$ ), "C $\mathrm{C}_{\mathrm{ds} 1}$ ", and "C $\mathrm{C}_{\mathrm{gs} 2}$ ". These parasitics form a feedforward (or bypass) path between the two transconductance stages. Hence for signals with frequency contents higher than the pole caused by these two capacitors, these signals would be "shorted" between the input and output nodes of the gyrator feedback loop; and the capacitance (or 
whatever load impedance) at node $v_{2}$ would be seen by test voltage at node $v_{1}$. In other words, the gyrator feedback loop ceases to perform the impedance transformation.

\subsection{3 "Common-source, Common-source" feedback loop}

A "Common-source" configuration is usually used as a transconductance gain stage. Since both transconductive elements are of the same "common-source" configuration, the only possible method to obtain both positive and negative transconductive elements in a feedback loop is to connect them with direct- and cross-connections between the two differential pair stages (i.e. see Figure 3.7). In addition, the need for differential pair stages (instead of single-ended "common-source" stage) arises from the fact that differential pair stages steer the bias current from one branch to the other in a complementary manner. Thus the cross-connection provides us the needed positive tranconductive behaviour out of a "common-source" stage.

In addition to fulfilling the requirement of having both positive and negative transconductive element implementation inside a gyrator-capacitor feedback loop, the "differential pair with tail current source" structure also provides a few circuit perfor- 
mance improvement. Specifically, the symmetrical property of differential circuit reduces its sensitivity of common-mode interference from input and power supply. Furthermore, the shared source node of a differential pair structure acts as a virtual ground for differential input signals. In other words, the body effect caused by the tail current source only has effect on the DC bias point of the differential pair, but not on the ac signal path. 


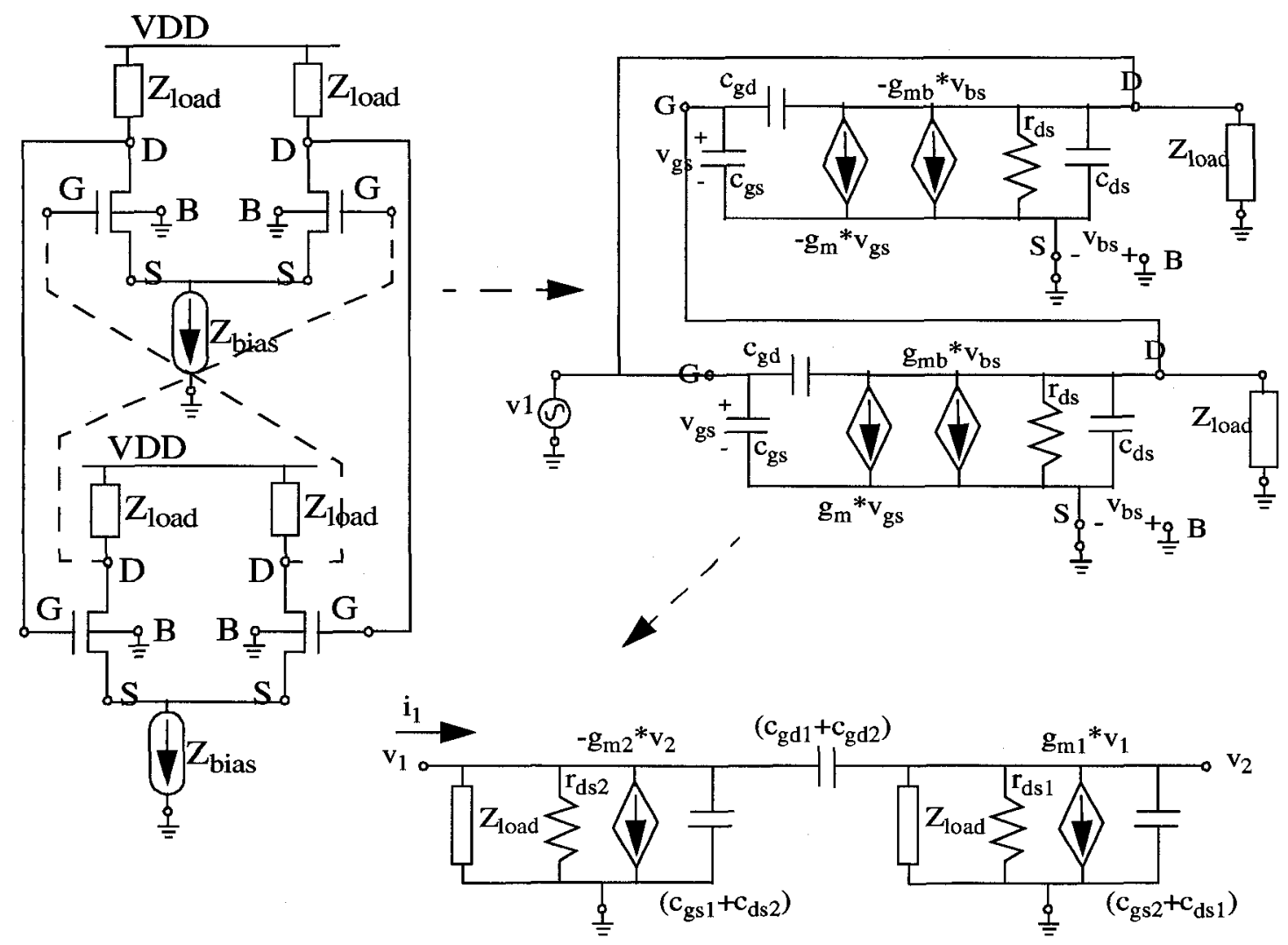

Figure 3.7: common-source/common-source feedback configuration and its small signal differential-half-circuit models

The impedance transformation (i.e. from capacitive loading to inductive loading) can be derived with small signal models of the above circuit and Kirchhoff's Current Law as follows: 
$\mathrm{KCL}$ at node $\mathrm{v}_{1}$ :

$i_{1}=\left[Y_{l o a d}+g_{d s 2}+s \cdot\left(C_{g s 1}+C_{d s 2}+C_{g d 1}+C_{g d 2}\right)\right] \cdot v_{1}-\left[g_{m 2}+s \cdot\left(C_{g d 1}+C_{g d 2}\right)\right] \cdot v_{2}$

where $\mathrm{i} 1$ is defined as the current going into node $\mathrm{v}_{1}, \mathrm{~g}_{\mathrm{ds} 1}=1 / \mathrm{r}_{\mathrm{ds} 1}, \mathrm{Y}_{\text {load }}=1 / \mathrm{Z}_{\mathrm{load}}$.

$\mathrm{KCL}$ at node $\mathrm{v}_{2}$ :

$$
g_{m 1} \cdot v_{1}+s \cdot\left(C_{g d 1}+C_{g d 2}\right) \cdot\left(v_{2}-v_{1}\right)+\left[g_{d s 1}+Y_{l o a d}+s \cdot\left(C_{d s 1}+C_{g s 2}\right)\right] \cdot v_{2}=0
$$

where $Y_{\text {load }}=1 / Z_{\text {load }}$ and $g_{d s 1}=1 / r_{d s 1}$.

After re-arranging and isolating the $\mathrm{v}_{1}$ and $\mathrm{v}_{2}$ terms in Equation 3.26:

$$
v_{2}=\frac{-g_{m 1}+s \cdot\left(C_{g s 1}+C_{g d 2}\right)}{g_{d s 1}+Y_{l o a d}+s \cdot\left(C_{d s 1}+C_{g d 1}+C_{g d 2}+C_{g s 2}\right)} \cdot v 1
$$

Substitute Equation 3.27 into the variable $v_{2}$ of Equation 3.25, and re-arrange for $\mathrm{i}_{1} / \mathrm{v}_{1}$ :

$$
\frac{i_{1}}{v_{1}}=Y_{\text {parallel }}+s \cdot\left(C_{\text {parallel }}\right)+\frac{1}{Z_{\text {series }}+s \cdot\left(L_{\text {transformed }}\right)}
$$


where

$$
\begin{gathered}
Y_{\text {parallel }}=Y_{l o a d}+g_{d s 2} \\
C_{\text {parallel }}=C_{g d 1}+C_{g s 1}+C_{d s 2}+C_{g d 2} \\
Z_{\text {series }}=\frac{Y_{l o a d}+g_{d s 2}}{\left(g_{m 1} \cdot g_{m 2}\right)+s \cdot\left(C_{g d 1}+C_{g d 2}\right) \cdot\left(g_{m 1}-g_{m 2}\right)-s^{2} \cdot\left(C_{d s 1}+C_{g s 2}\right)^{2}}
\end{gathered}
$$

and $\mathrm{L}_{\text {transformed }}$ represents the transformed inductance, as follows:

$$
L_{\text {transformed }}=\frac{C_{d s 1}+C_{g d 1}+C_{g d 2}+C_{g s 2}}{\left(g_{m 1} \cdot g_{m 2}\right)+s \cdot\left(C_{g d 1}+C_{g d 2}\right) \cdot\left(g_{m 1}-g_{m 2}\right)-s^{2} \cdot\left(C_{d s 1}+C_{g s 2}\right)^{2}}
$$

Equations 3.28 to 3.32 represent the transformed admittance and impedance by the gyrator-capcitor feedback circuit (implemented with a common-source stage, followed by a common-source stage). $Y_{\text {parallel }}$ (of Equation 3.29) and $C_{\text {parallel }}$ (of Equation 3.30) are the admittance and capacitance seen between the input node $\mathrm{v}_{1}$ and ground. $\mathrm{Z}_{\text {series }}$ (of Equation 3.31) is the series resistance of the transformed inductance, whereas $L_{\text {transformed }}$ (of Equation 3.32) is the transformed inductance generated by the gyrator-capacitor feedback circuit. 
In the denominator polynominal (of Equations 3.31 and 3.32), the $\mathrm{s}^{2}$ term becomes negligible, which is due to the product of our target oscillation frequency and the parasitic capacitance (i.e. range of giga-Hertz multiplied by femto-Farad, then squared). In addition, if both "common-source" stages are biased to have identical $g_{m}$ values; then the $s$ term equals to zero (i.e. $g_{m} 2^{-g_{m}}$ ). Hence the denominator polynominal will be simplified to $\mathrm{g}_{\mathrm{m} 1}{ }^{*} \mathrm{~g}_{\mathrm{m} 2}$, which is similar to the denominator of both $\mathrm{Z}_{\text {series }}$ and $\mathrm{L}_{\text {transformed }}$ terms in Equation 3.4.

Similar to both "common-drain, common-source" and "common-gate, commonsource" gyrator-capacitor feedback circuit topologies, the extra components that exist in the "common-source, common-source" feedback circuit belong to the feedforward (or bypass) path across both transconductive elements. In this case, these "bypass" paths are formed by parasitic capacitors $\mathrm{C}_{\mathrm{gd}}$ of both transconductive elements.

\subsubsection{Summary of " $g_{m}$ " and " $-g_{m}$ " implementation in CMOS}

This section summarizes the results of the gyrator-capacitor feedback circuit implementations described in previous sub-sections. 
TABLE 3.1. Summary of "gm" and "-gm" implementation in CMOS technology

\begin{tabular}{|c|c|c|c|c|c|}
\hline & $\mathbf{Y}_{\text {parallel }}$ & $\mathbf{C}_{\text {parallel }}$ & $\mathbf{Z}_{\text {series }}$ & $\mathbf{L}_{\text {transformed }}$ & $\begin{array}{l}\text { bypass } \\
\text { components }\end{array}$ \\
\hline ideal & $1 / \mathrm{r}_{\mathrm{D} 2}$ & $\mathrm{C}_{\mathrm{i} 1}+\mathrm{C}_{\mathrm{o} 2}$ & $1 /\left(\mathrm{r}_{\mathrm{o} 1}{ }^{*} \mathrm{~g}_{\mathrm{m} 1}{ }^{*} \mathrm{~g}_{\mathrm{m} 2}\right)$ & $\begin{array}{l}\left(\mathrm{C}_{\mathrm{o} 1}+\mathrm{C}_{\mathrm{i} 2}+\mathrm{C}_{\mathrm{load}}\right) / \\
\left(\mathrm{g}_{\mathrm{m} 1}{ }^{*} \mathrm{~g}_{\mathrm{m} 2}\right)\end{array}$ & N/A \\
\hline CD-CS & $\mathrm{Y}_{\text {load }}+\mathrm{g}_{\mathrm{ds} 2}$ & $\begin{array}{l}\mathrm{C}_{\mathrm{gd} 1}+\mathrm{C}_{\mathrm{gs} 1}+ \\
\mathrm{C}_{\mathrm{ds} 2}+\mathrm{C}_{\mathrm{gd} 2}\end{array}$ & $\begin{array}{l}\left(\mathrm{g}_{\mathrm{m} 1}+\mathrm{g}_{\mathrm{mb} 1}+\mathrm{g}_{\mathrm{ds} 1}\right. \\
+\mathrm{Y}_{\text {load }} / \mathrm{D}_{\mathrm{CDCS}}\end{array}$ & $\begin{array}{l}\left(\mathrm{C}_{\mathrm{ds} 1}+\mathrm{C}_{\mathrm{gs} 1}+\mathrm{C}_{\mathrm{gd} 2}+\right. \\
\left.\mathrm{C}_{\mathrm{gs} 2}\right) / \mathrm{D}_{\mathrm{CDCS}}\end{array}$ & $\mathrm{C}_{\mathrm{gs} 1}, \mathrm{C}_{\mathrm{gd} 2}$ \\
\hline CG-CS & $\begin{array}{l}\mathrm{g}_{\mathrm{m} 1}+\mathrm{g}_{\mathrm{mb} 1}+ \\
\mathrm{g}_{\mathrm{ds} 1}+\mathrm{g}_{\mathrm{ds} 2}\end{array}$ & $\begin{array}{l}\mathrm{C}_{\mathrm{ds} 1}+\mathrm{C}_{\mathrm{gs} 1}+ \\
\mathrm{C}_{\mathrm{ds} 2}+\mathrm{C}_{\mathrm{gd} 2}\end{array}$ & $\begin{array}{l}\left(\mathrm{Y}_{\mathrm{load}}+\mathrm{g}_{\mathrm{ds} 2}\right) / \\
\mathrm{D}_{\mathrm{CGCS}}\end{array}$ & $\begin{array}{l}\left(\mathrm{C}_{\mathrm{ds} 1}+\mathrm{C}_{\mathrm{gd} 1}+\mathrm{C}_{\mathrm{gd} 2}+\right. \\
\left.\mathrm{C}_{\mathrm{gs} 2}\right) / \mathrm{D}_{\mathrm{CGCS}}\end{array}$ & $\mathrm{C}_{\mathrm{ds} 1}, \mathrm{C}_{\mathrm{gd} 2}$ \\
\hline CS-CS & $\mathrm{Y}_{\text {load }}+\mathrm{g}_{\mathrm{d} \mathrm{s} 2}$ & $\begin{array}{l}\mathrm{C}_{\mathrm{gd} 1}+\mathrm{C}_{\mathrm{gs} 1}+ \\
\mathrm{C}_{\mathrm{ds} 2}+\mathrm{C}_{\mathrm{gd} 2}\end{array}$ & $\begin{array}{l}\left(\mathrm{Y}_{\text {load }}+\mathrm{g}_{\mathrm{ds} 2}\right) / \\
\mathrm{D}_{\mathrm{CSCS}}\end{array}$ & $\begin{array}{l}\left(\mathrm{C}_{\mathrm{gd} 1}+\mathrm{C}_{\mathrm{gs} 1}+\mathrm{C}_{\mathrm{ds} 2}+\right. \\
\mathrm{C}_{\mathrm{gd} 2} / \mathrm{D}_{\mathrm{CSCS}}\end{array}$ & $\mathrm{C}_{\mathrm{gd} 1}, \mathrm{C}_{\mathrm{gd} 2}$ \\
\hline
\end{tabular}

where

CD-CS represents "common-drain, common-source" configuration

CG-CS represents "common-gate, common-source" configuration

CS-CS represents "common-source, common-source" configuration

$\mathrm{D}_{\mathrm{CDCS}}$ is the polynomial in the denominator of Equations 3.11 and 3.12

$\mathrm{D}_{\mathrm{CGCS}}$ is the polynomial in the denominator of Equations 3.22 and 3.23

$\mathrm{D}_{\mathrm{CSCS}}$ is the polynomial in the denominator of Equations 3.31 and 3.32

" $1 / \mathrm{r}_{\mathrm{o} 1}$ " and " $1 / \mathrm{r}_{\mathrm{o} 2}$ " in the ideal case are similar to " $\mathrm{g}_{\mathrm{ds} 1}$ " and " $\mathrm{g}_{\mathrm{ds} 2}$ " of a MOS device

Among all three gyrator-capacitor feedback loop configurations, the "commonsource, common-source" feedback configuration is the closest implementation to the ideal 
case presented in Section 3.1. Thus "common-source" configured NMOS devices are utilized as both "gm" and "-gm" transconductive amplifiers in the implemented gyratorcapacitor feedback loop circuit. Furthermore, this feedback circuit is also the basis of an active-inductor-capacitor voltage controlled oscillator; which will be covered in the next section.

\subsection{Active-inductor-capacitor voltage controlled oscillator}

This section covers the implementation of an active inductor-capacitor voltage controlled oscillator circuit, which is based from the gyrator-capcitor feedback circuit described in previous section.

The active-inductor-capacitior voltage controlled oscillator (or active LC-VCO) circuit is built upon the architecture of a passive inductor capacitor voltage controlled oscillator circuit; which consists of a lossy tuned LC resonant circuit connected in parallel with a gain amplifier. Main differences between the passive and active LC-VCO circuit implementations are summarized as follows: 
1. the fixed value passive inductor is replaced by a tunable active inductor, which is generated by a gyrator-capacitor feedback loop circuit

2. the tunable varactor is replaced by parasitic capacitances within the gyrator-capacitor feedback loop circuit

3. complementary "-gm" gain stages are replaced with NMOS "-gm" gain stages

4. bias currents from the power supply to active inductors and NMOS "-gm" gain stages is accomplished with loads, which is due to the absense of PMOS devices inside the "gm" gain stages

From the "common-source, common-source" gyrator-capacitor feedback circuit, losses exist due to both $\mathrm{Y}_{\text {parallel }}$ and $\mathrm{Z}_{\text {series }}$ elements of the non-ideal inductor model (Equations 3.29 and 3.31); hence positive feedback latches are connected in shunt with each transconductive stage to compensate for these losses (see Figure 3.8). These positive feedback latches are equivalent to the "-gm" gain cells in a passive LC-VCO circuit topology.

Due to the symmetrical property of the "common-source, common-source" feedback topology, impedance transformation is bidirectional, namely from capacitive loading 
at node v2 (or node v1) to inductive loading behaviour at node v1 (or node v2) respectively. Therefore each of the two positive feedback latches is connected to two different active inductors. The tail current sources of the two positive feedback latches are implemented to match the DC bias voltage levels between the active inductor stages and the latches. In addition, these current sources are part of the frequency tuning control mechanism; which will be described in the next section.
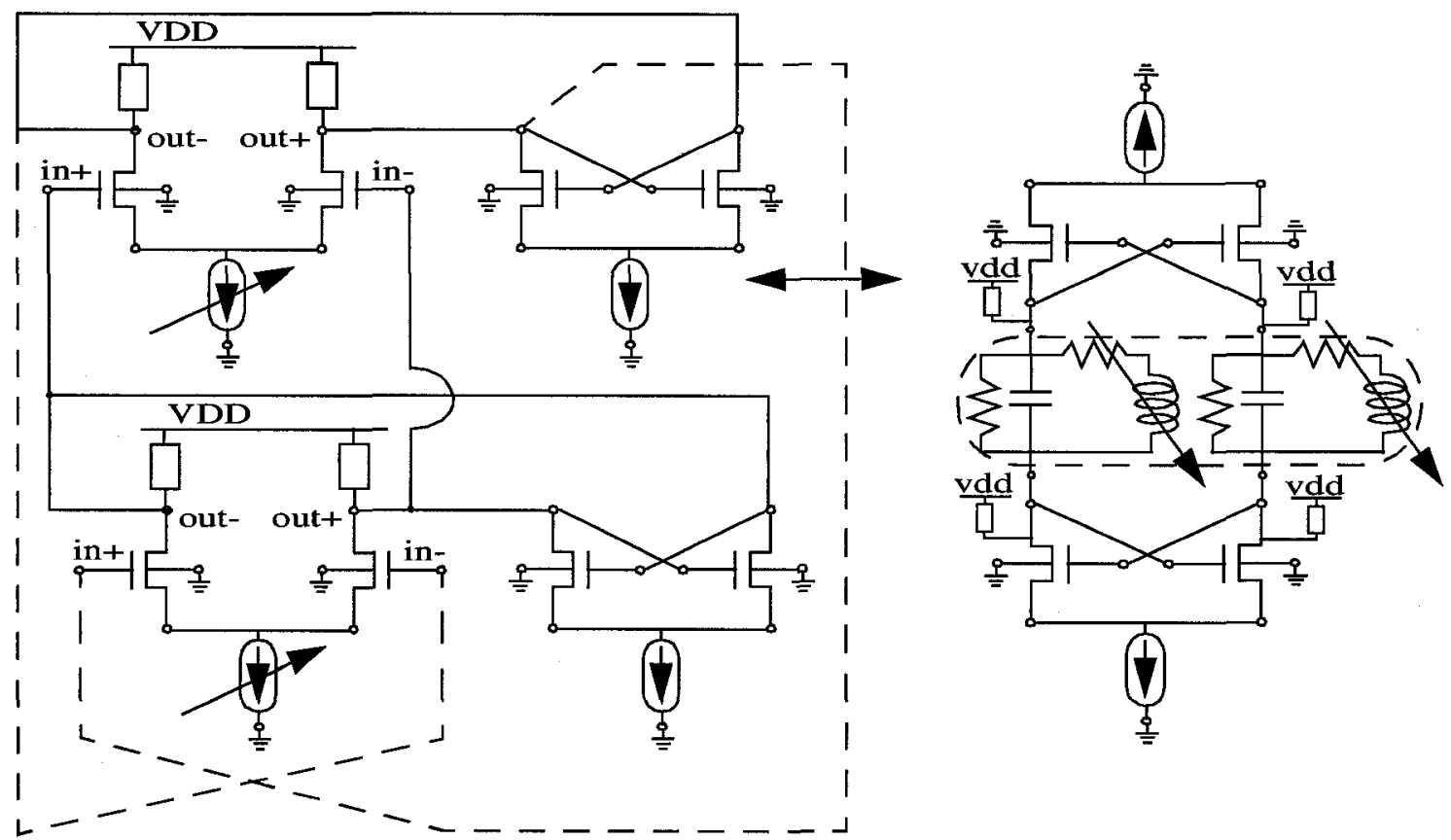

Figure 3.8: Conceptual active LC-VCO circuit and its tunable inductor model 
The following sub-sections cover the implementation of the proposed active inductor capacitor voltage controlled oscillator circuit.

\subsubsection{Implementation of an active-inductor based oscillator circuit}

The active-inductor based voltage-controlled-oscillator circuit consists of a bias circuit, a voltage-to-current converter circuit, and a 2-stage current-controlled-oscillator circuit (see Figure 3.9). In order to send oscillator output signals to an off-chip environment, a 3-stage buffer circuit is placed between the oscillator circuit and the on-chip bondwire pads. 


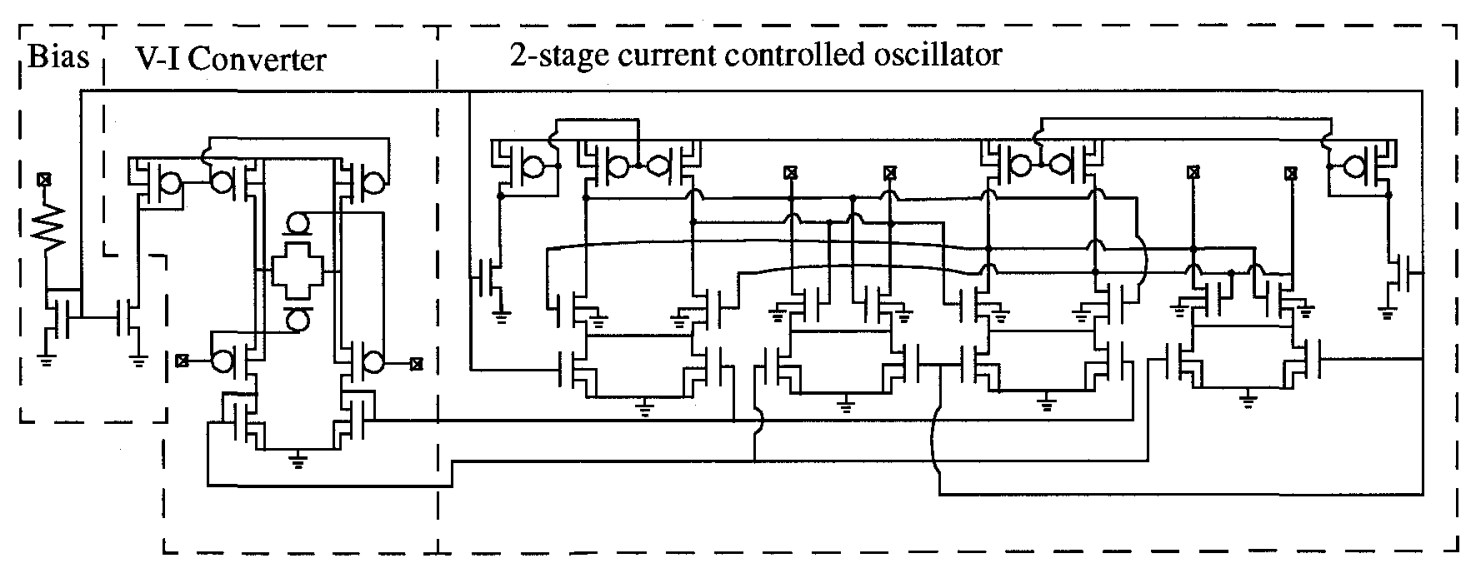

Figure 3.9: schematic of active LC-VCO circuit with bias and frequency tuning circuits

\subsubsection{Bias Circuit Topology}

The bias circuit provides bias for both the voltage-current-converter and currentcontrolled-oscillator circuits. The bias circuit consists of a series-connected p+ poly resistor placed between the input pad and the NMOS current mirror structure.

The series resistor is formed by two shunt-connected resistors of $811.304 \mathrm{ohm}$, with width and length of $0.92 \mathrm{um}$ and $2.4 \mathrm{um}$ respectively. The NMOS current mirror has a 
ratio of $8: 4: 12: 16: 4: 16: 4: 12$, with transistor width and length of 2.5 um and 0.4 um respectively (see Figure 3.10).

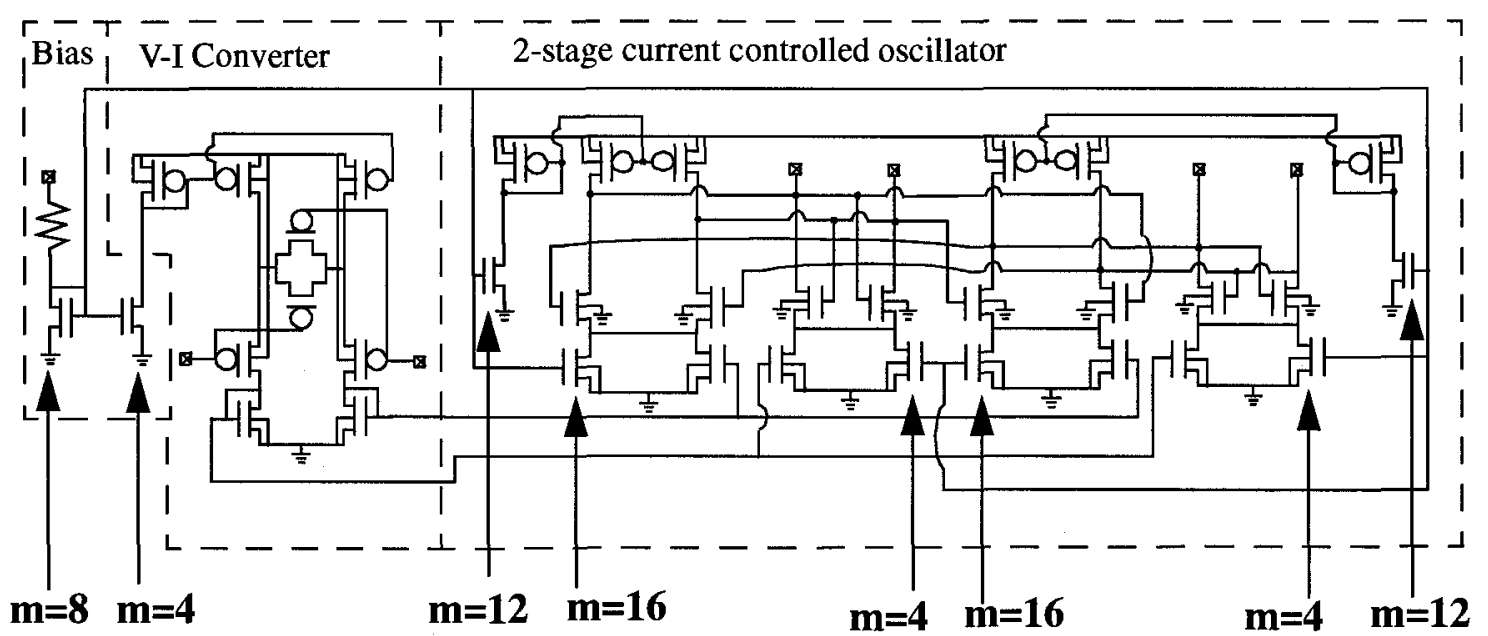

Figure 3.10: active LC-VCO circuit bias scheme (fixed oscillator current)

The first current mirror ratio of $8: 4$ is used to set the bias current for the voltage-tocurrent converter circuit. The second and seventh current mirror ratios of $8: 12$ are used to set the bias current for the PMOS active load (another current mirror) of each of the oscillator circuit stage. The third and fifth current mirror ratios of 8:16 are used to set the "fixed" amount of bias current for the NMOS tail current of each of the differential pair stages (part of the oscillator output frequency tuning circuit). The fourth and sixth current 
mirror ratios of 8:4 are used to set the "fixed" amount of bias current for the positive feedback latch stage (another part of the oscillator output frequency tuning circuit).

\subsubsection{Voltage-to-Current Converter Circuit Topology}

The voltage-to-current converter circuit consists of a PMOS differential pair stage, which steers the bias current to one of two output nodes.

In order to provide a relatively linear voltage to current conversion, the differential pair is implemented with PMOS transistors as degeneration; which operate in triode operation mode. The bias current of such a differential pair gain stage is supplied by a PMOS current mirror, whose reference current comes from the bias circuit.

The diode-connected active loads of this PMOS differential pair stage are the input sides of two NMOS current mirrors, which are used to control the bias current of the current-controlled-oscillator circuit and its output frequency.

The PMOS current mirror has a ratio of 8:4:4, with device width and length of 5um and 0.4um respectively. The PMOS differential pair stage consists of device width/ 
length/finger(s) of $2.5 \mathrm{um}, 0.2 \mathrm{um}$, and 8 respectively. The PMOS degeneration devices are sized with width/length/finger(s) of $2.5 \mathrm{um}, 0.2 \mathrm{um}$, and 2 respectively (see Figure 3.11 ).

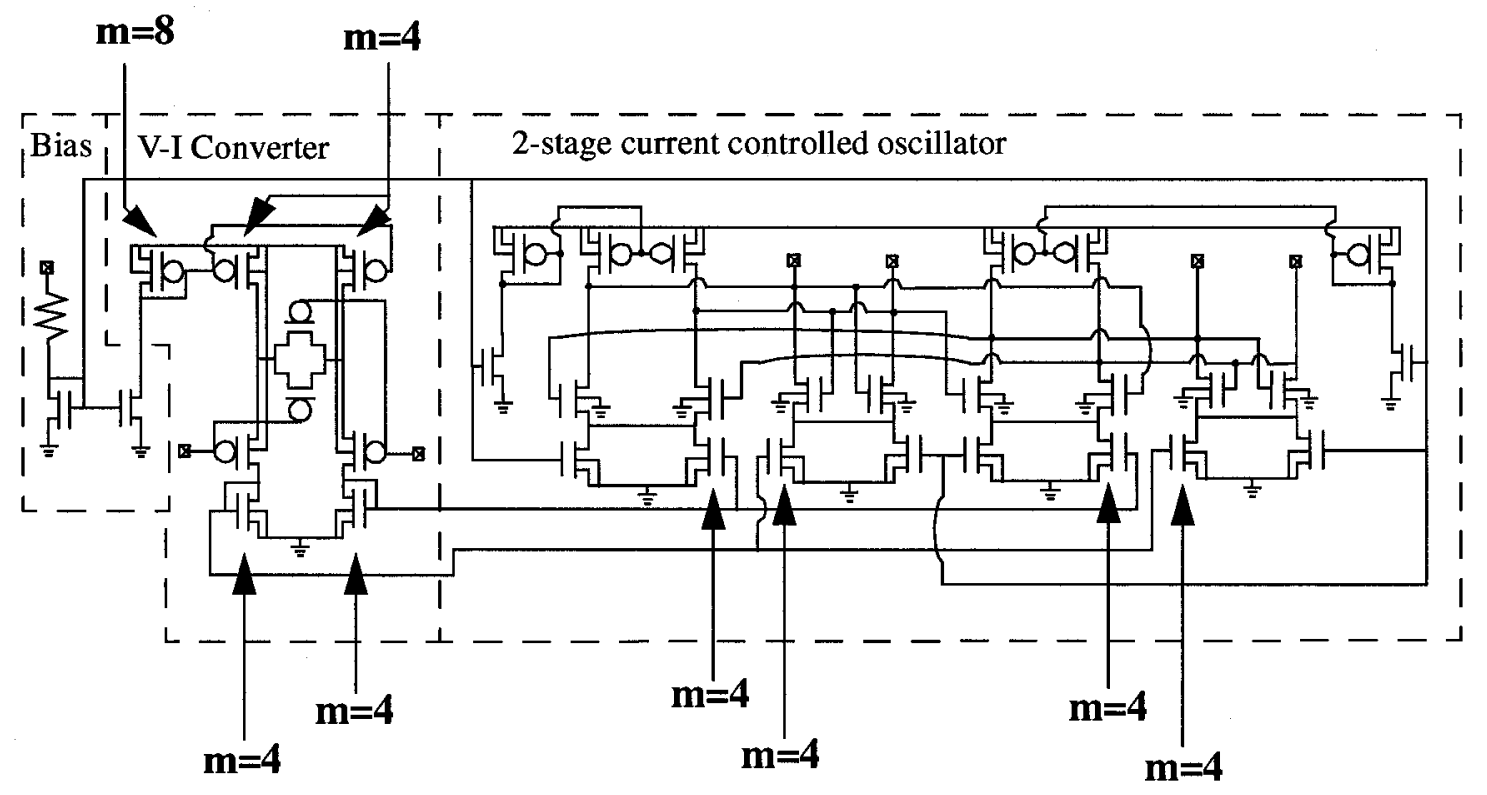

Figure 3.11: active LC-VCO circuit output frequency tuning (bias scheme)

The NMOS active loads are designed as two current mirrors of ratios 4:4:4; their devices have width and length of 2.5um and 0.4um respectively (see Figure 3.11). These two current mirrors provide the "variable" portion of bias current of the 2-stage currentcontrolled-oscillator circuit. 


\subsubsection{Current-Controlled-Oscillator Circuit Topology}

From Figure 3.9, the 2-stage current-controlled-oscillator circuit is based on the active inductive-capacitive voltage-controlled-oscillator (or active LC-VCO) circuit topology. The name "current-controlled-oscillator" is a more appropriate description of its current-controlled frequency tuning mechanism.

Each of the two identical stages of oscillator circuit consists of a differential pair in shunt connection with a positive feedback latch. The bias currents of both stages of oscillator circuits consist of a "fixed" and a "variable" portion for frequency tuning (see Figures 3.10 and 3.11). The loads of each oscillator stage are made of separate PMOS current mirrors, whose bias current doesn't change with the frequency tuning scheme. The overall oscillator device sizing are summarized as follows:

$\begin{array}{llll}\text { Differential pair NMOS } & \mathrm{W}=1.25 \mathrm{um} & \mathrm{L}=0.18 \mathrm{um} & \mathrm{m}=12: 12 \\ \text { Positive feedback NMOS } & \mathrm{W}=1.25 \mathrm{um} & \mathrm{L}=0.18 \mathrm{um} & \mathrm{m}=4: 4 \\ \text { Load (current mirror) PMOS } & \mathrm{W}=1.25 \mathrm{um} & \mathrm{L}=0.2 \mathrm{um} & \mathrm{m}=12: 12: 12\end{array}$




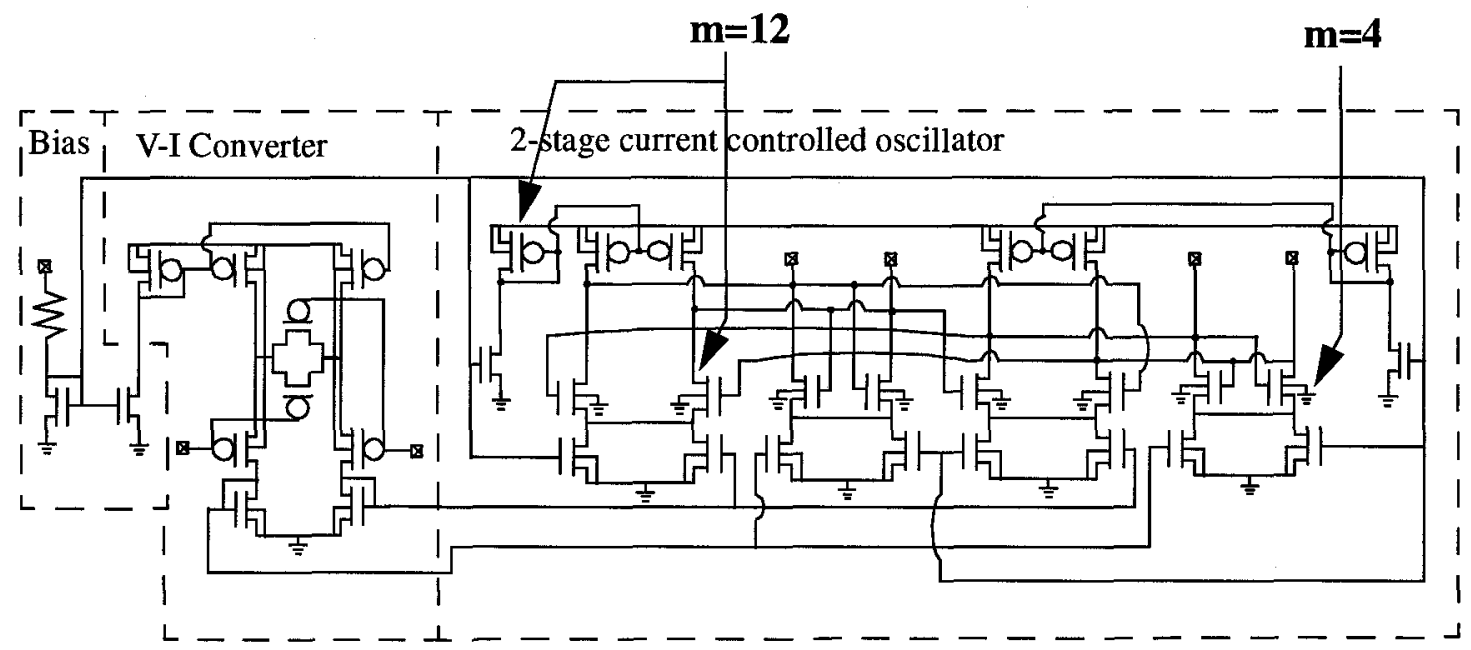

Figure 3.12: active LC-VCO circuit device sizing

The decision to use current mirror active loads instead of resistive loads is because of their shunt connection to both the differential pair and latch circuits. Since the differential pair stage and the latch circuits represent the active inductive-capacitive load and the "-gm" gain stage of the oscillator respectively, a high impedance "shunt-connected" load is chosen to minimize the degeneration of the quality factor of the overall circuit. For high speed operation, the NMOS transistors of both differential pair stages operate in either "cutoff" or "saturation" mode. Hence the maximum peak-to-peak output signal swing of 
the oscillator circuit must be less than or equal to the threshold voltage of the differential pair NMOS transistors.

\subsubsection{Output Buffer Circuit Topology}

A 3-stage output buffer is built to drive the oscillator output signals to an off-chip environment. The output buffer circuit consists of two consecutive stages of differential pair circuits, followed by a common-drain circuit as the final stage of output driver (see Figure 3.13). The device sizing are summarized as follows:

\begin{tabular}{|c|c|c|c|}
\hline Bias NMOS & $\mathrm{W}=5 \mathrm{um}$ & $\mathrm{L}=0.4 \mathrm{um}$ & $\mathrm{m}=4: 8: 12$ \\
\hline 1st Differential Pair NMOS & $\mathrm{W}=1.5 \mathrm{um}$ & $\mathrm{L}=0.18 \mathrm{um}$ & $\mathrm{m}=4$ \\
\hline 1st Differential Pair Resistor & $\mathrm{W}=920 \mathrm{~nm}$ & $\mathrm{~L}=2.4 \mathrm{um}$ & $\mathrm{R}=1216.956 \mathrm{ohm}$ \\
\hline 2nd Differential Pair NMOS & $\mathrm{W}=1.5 \mathrm{um}$ & $\mathrm{L}=0.18 \mathrm{um}$ & $\mathrm{m}=8$ \\
\hline 2nd Differential Pair Resisto & $\mathrm{W}=920 \mathrm{~nm}$ & $\mathrm{~L}=2.4 \mathrm{um}$ & $\mathrm{R}=405.652 \mathrm{ohm}$ \\
\hline 3rd Common Drain NMOS & $\mathrm{W}=2.5 \mathrm{um}$ & $\mathrm{L}=0.18 \mathrm{um}$ & $m=32$ \\
\hline
\end{tabular}




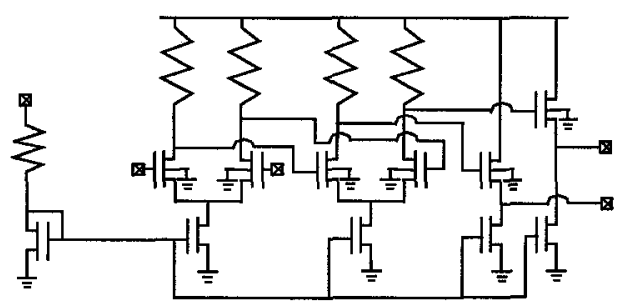

\section{Figure 3.13: 3-stage buffer circuit}

The objectives of this output buffer circuit include:

1. To provide a buffer between the on-chip oscillator circuit and an off-chip low impedance environment. Hence the oscillator circuit can achieve higher oscillation frequency with less amount of parasitic loading from the off-chip environment. In other words, the only extra capacitive loading for the oscillator circuit will be the first stage of the buffer circuit.

2. To provide a relatively low output impedance with respect to the off-chip environment. Therefore, oscillator circuit output signals can be transferred to the external load (i.e. probe equipment) without much attentuation.

3. For matching purpose, the $1216.956 \mathrm{ohm}$ and $405.652 \mathrm{ohm}$ resistors (of first 2 buffer circuit stages) are created with a "unit" resistor of $811.304 \mathrm{ohm}$. This is part of the device matching strategy from the layout perspective. 


\subsubsection{Similarity with frequency divider circuit}

The 2-stage voltage controlled oscillator circuit is very similar to a divide-by-2 circuit, which consists of 2 differential source coupled latches connected in a negative feedback configuration (i.e. negative output node of the second latch is connected to the positive input node of the first latch). The only difference lies in the removal of the clock input MOS differential pair circuits, which are usually placed between the data input MOS differential pair and the bias circuits (see Figures 3.12 and 3.14).

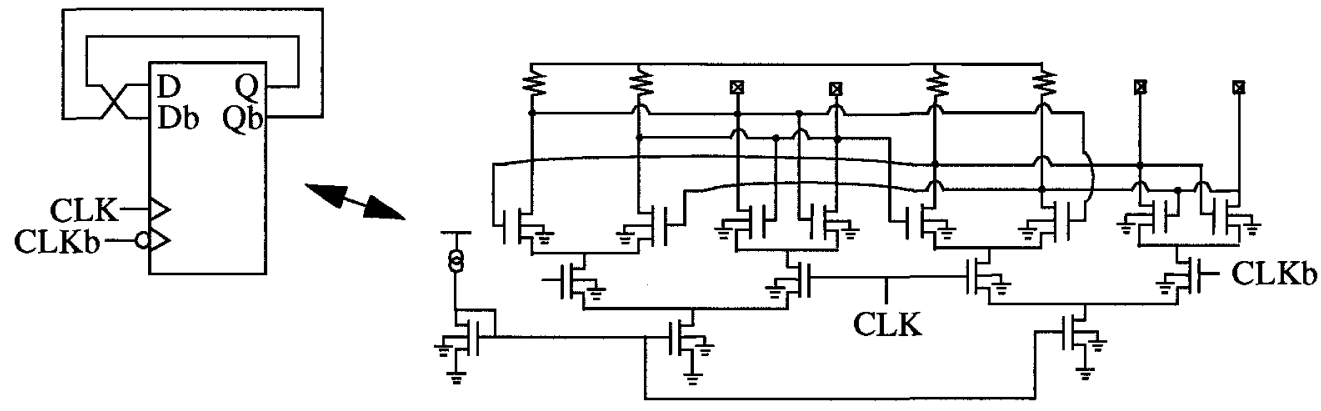

Figure 3.14: schematic of a divided-by-2 circuit with top level and transistor level views 
Alternately, the 2-stage voltage controlled oscillator circuit can be implemented with a divide-by-2 structure; provided the clock input MOS devices are biased with fixed DC voltages and operate in "saturation" mode.

\subsection{Active LC-VCO circuit operation mechanism}

This section covers the operation mechanism of the implemented active inductor capacitor voltage controlled oscillator circuit. Specifically, the operating principle of a 2stage VCO circuit will be covered in the first half of this section; whereas the frequency tuning mechanism of this VCO circuit will be covered in the second half of this section.

As demonstrated in Chapter 2, that all feedback circuits with self-sustaining oscillation must follow the "Barkhausen Criterion", which is summarized as follows:

$$
|G(s)|=1 \leftrightarrow \angle G(s)=0
$$

Hence for a feedback circuit with an odd number of inverting stages (i.e. $\mathrm{N}$ singleended inverter staged ring oscillator), one oscillation period is completed when a signal propagates through all $\mathrm{N}$ stages twice (see Figure 3.15). In other words, a total of 360- 
degree of phase shift occurs after the signal has propagated through all $\mathrm{N}$ stages twice. Consequently, a total of 180-degree of phase shift must be distributed evenly among $\mathrm{N}$ inverting stages.
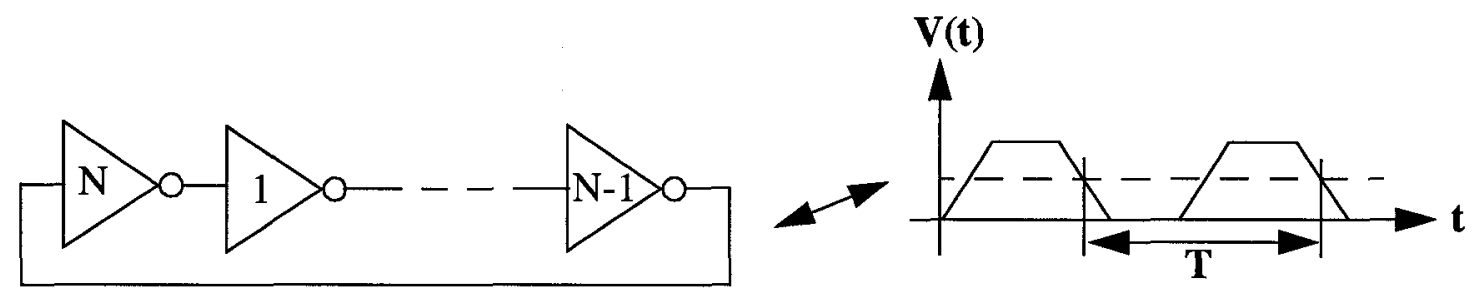

Figure 3.15: ring oscillator model with $\mathbf{N}$ stages of single-ended inverters

In the case of a feedback circuit consists of differential inverting stages, the number of stages can be even or odd; as long as the total number of inversion within the feedback loop is an odd number. Thus the aforementioned condition of 180-degree phase shift distribution among all stages still holds.

For instance, the proposed active LC-VCO circuit can be interpretted as a 2-stage ring oscillator. Hence there will be a 90-degree phase shift between each inverting stage (see Figure 3.16). 


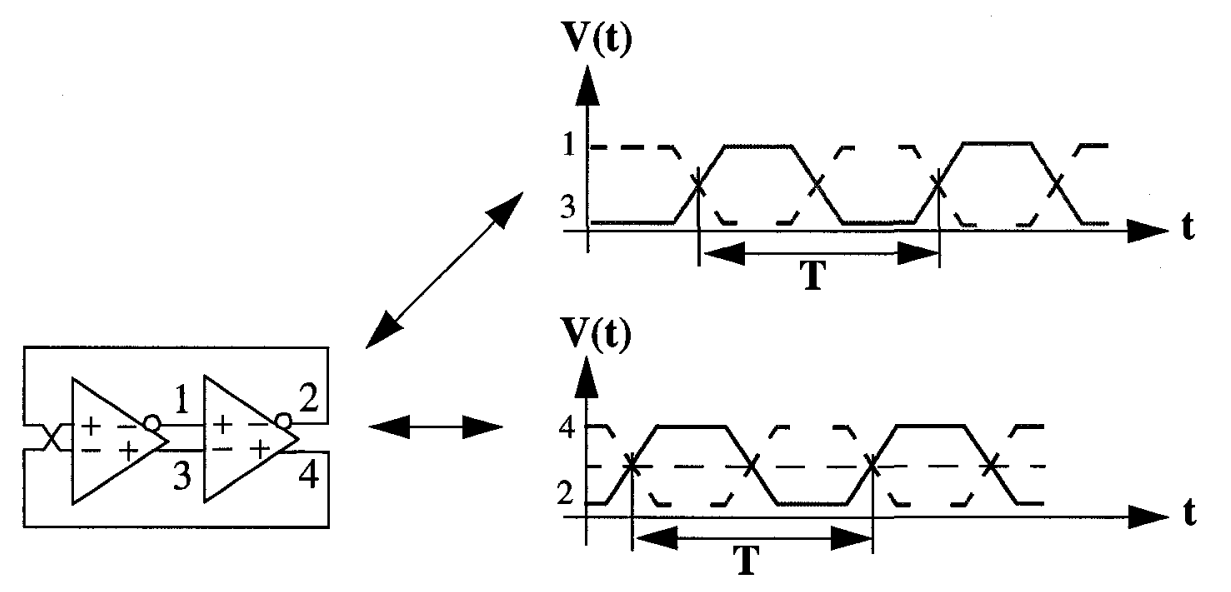

Figure 3.16: A 2-stage ring oscillator and its output waveform model

\subsubsection{Frequency tuning of current controlled oscillator (small signal analysis)}

From Equation 3.32, the transformed inductance value decreases as the product of the two transconductive elements " $\mathrm{g}_{\mathrm{m} 1}$ " and " $\mathrm{g}_{\mathrm{m} 2}$ " increases (i.e. with increase in tail current of elements " $\mathrm{g}_{\mathrm{m} 1}$ ' and ' $\mathrm{g}_{\mathrm{m} 2}$ "). Since the capacitive loadings on the inductive nodes are fixed upon the completion of the circuit layout, the oscillation frequency of the acitive LC-VCO increases as the bias currents of the two transconductive elements increase. 
Since both differential pair (i.e. transconductive elements " $\mathrm{g}_{\mathrm{m} 1}$ " and " $\mathrm{g}_{\mathrm{m} 2}$ ") and positive-feedback latch circuits share the same load in the active LC-VCO architecture (see Figure 3.8), the proper way to tune the oscillation frequency is to steer a small amount of bias current between the two types of circuits. Hence the total amount of bias and tuning current will go through the same load, and a constant oscillation amplitude will be maintained.

\subsubsection{Frequency tuning of current controlled oscillator (large signal analysis)}

As mentioned in Section 3.3.6, the proposed two stage current controlled oscillator is very similar to a divide-by- 2 circuit; which is composed of a negative-feedback connection around two cascading differential source-coupled latch circuits. Data propagation through each latch circuit is enabled/disabled by the differential pair and the latch circuits respectively; which is controlled by the clock input differential pair circuits. Based on the current steering principle of a differential pair circuit, one can control the data propagation by varying the bias current of the data level differential pair and latch circuits. 
If the clock differential pair circuit is replaced with separate bias current control circuits, the propagation of data within the D-flip-flop is also an oscillation frequency tuning mechanism for the divide-by- 2 circuit. The fastest oscillation speed occurs when the two latch circuits are inoperative, and "data" signal propagates around the D-flip-flop via two differential pair circuits. Likewise, the slowest oscillation speed (i.e. not oscillating) can be achieved if all bias currents flow through the two latches instead of the two differential pair circuits. Thus, this is the strategy employed in the proposed two stage current controlled oscillator circuit (see Figure 3.11)

In order to ensure proper oscillation without latch-up, more bias current is supplied to the differential pair circuits than the latches; and the bias current for the voltageto-current converter circuit is split between the differential pair and latch circuits for frequency tuning purpose.

\subsection{Active LC-VCO noise sources}

This section covers the potential noise sources found in the proposed active LCVCO circuit; namely the PMOS active load, the NMOS switching pairs, the NMOS cross- 
coupled latches, and the bias tail current NMOS device (see Figures 3.10, 3.11, and 3.12). Diagrams and equations are reproduced from one of Hajimiri's papers published in May of 1999 [10]; but with modification made for the proposed 2-stage active LC-VCO circuit.

Within each oscillator delay stage, the total differential noise power from the two NMOS switching-pair and cross-coupled-latch devices are summarized as follows:

$$
\overline{i_{\text {core }}{ }^{2}}=\frac{1}{4} \cdot\left(\overline{i_{n 1}{ }^{2}}+\overline{i_{n 2}{ }^{2}}+\overline{i_{n 3}{ }^{2}}+\overline{i_{n 4}{ }^{2}}\right)=\left(\overline{i_{n}^{2}}\right)
$$

where " $\mathrm{i}_{\mathrm{n}}{ }^{2}$ " is the equivalent current noise sources of each NMOS device.

The noise spectral densities of the NMOS switching/cross-coupled-latch and the PMOS active load devices are:

$$
\overline{\frac{i_{n}^{2}}{\Delta f}}=4 k T \gamma \mu_{n} C_{o x} \cdot\left(\frac{W_{n}}{L_{n}}\right) \cdot\left(V_{G S}-V_{T N}\right)
$$

and

$$
\overline{i_{p}^{2}} \frac{\Delta f}{\Delta f}=4 k T \gamma \mu_{p} C_{o x} \cdot\left(\frac{W_{p}}{L_{p}}\right) \cdot\left(V_{G S}-V_{T P}\right)
$$


where the value for " $\gamma$ " is about $2 / 3$ for long-channel MOS devices; and between 2 and 3 for short-channel MOS devices. The increase in the " $\gamma$ " value is due to hot-electron effects inside the "short" channel [10].

Since all NMOS switching-pair and cross-coupled-latch devices are part of the oscillation signal propagation path, both gate and drain nodes of these devices exhibit signals with ratioed power levels at odd and even harmonics of the output oscillation frequency. Noises close to these oscillation harmonic frequencies are down-converted as close-in phase noise at the oscillator circuit output spectrum. The mechanism of these frequency down-conversions will be covered in Chapter 5 .

In our proposed active LC-VCO circuit, each 1: $\mathrm{m}_{\mathrm{p}}$ PMOS and $1: \mathrm{m}_{\mathrm{n}}$ NMOS current mirror supplies the needed current to both NMOS switching-pair and cross-coupledlatch devices. Hence the current noise power from both NMOS tail current and PMOS active load devices can be summarized as:

$$
\overline{i_{\text {tail }}^{2}}=\overline{i_{b i a s}^{2}} \cdot m_{n}^{2}
$$

and 


$$
\overline{i_{\text {load }}{ }^{2}}=\overline{i_{\text {bias }}{ }^{2}} \cdot m_{p}^{2}
$$

which reveals the choice of the bias current mirror ratios has a significant impact on the noise contribution (see Figures 3.10,3.11, and 3.12 for the current bias schemes).

Similar to the NMOS switching-pair and cross-coupled-latch devices, the drain nodes of the PMOS active-load devices are also part of the oscillation signal propagation path; hence device noise near odd and even harmonics of the oscillation output frequency undergo down-conversion and become close-in phase noise at the oscillator output spectrum.

Since drain nodes of both NMOS current bias and frequency tuning devices (i.e. part of separate NMOS current mirror circuits) are connected to the source nodes of both NMOS switching-pair and cross-coupled latch devices, even harmonics of the oscillation frequency are found to be dominant at this connection. Hence noise near these even oscillation frequency harmonics undergo down-conversion and become phase noise at the oscillator circuit output spectrum. 
Although the current noise sources have similar behaviour as the proposed passive LC-VCO circuit, the additional number of current mirrors utilized for the proposed active LC-VCO circuit provide an even larger impact to the oscillator phase noise performance. More information related to device noise sources and oscillator phase noise performance will be discussed in Chapter 5 .

\subsection{Active LC-VCO Summary}

Based from the concept of active inductance generation through gyrator-capacitor feedback circuits, an active LC-VCO circuit has been proposed for this thesis research. The oscillator circuit mainly consists of three parts: a bias circuit to maintain minimum oscillation frequency, a voltage-to-current converter to perform frequency tuning, and a 2stage current controlled oscillator circuit that evolves from the gyrator-capacitor feedback circuit topology.

In the following chapter, layout technique on both active/passive LC-VCO circuits and an off-chip test structure are illustrated. In addition, test equipments for both oscillator circuits are summarized at the end of next chapter. 
This chapter covers layout technique of the two implemented oscillator circuits, and is divided into three sections. The first section covers some layout technique for the on-chip oscillator cirucit. The second section covers the off-chip test board and related wirebonding diagrams for both active and passive LC-VCO circuits. The third section covers the test equipment used in the lab.

\subsection{Passive-Inductor-Capacitor VCO Circuit Layout}

As described in the LC-VCO chapter (Chapter 2), the passive-inductor-capacitor oscillator core circuit consists of an on-chip differential spiral inductor, pn-junction varactors, and a negative-gm gain stage built with 2 CMOS inverter connected in positive feed- 
back manner. Since we have to drive the signal off-chip, there is a buffer circuit added in series between the LC-VCO core circuit and the output pads.

\subsubsection{On-chip Differential Spiral Inductor Layout}

The dimension of the on-chip differential spiral inductor has been defined and optimized by ASITIC. In the current design, the dimension of the implemented inductor is: $\mathrm{L}=200 \mathrm{um}, \mathrm{W}=14 \mathrm{um}, \mathrm{S}=3 \mathrm{um}, \mathrm{N}=3$

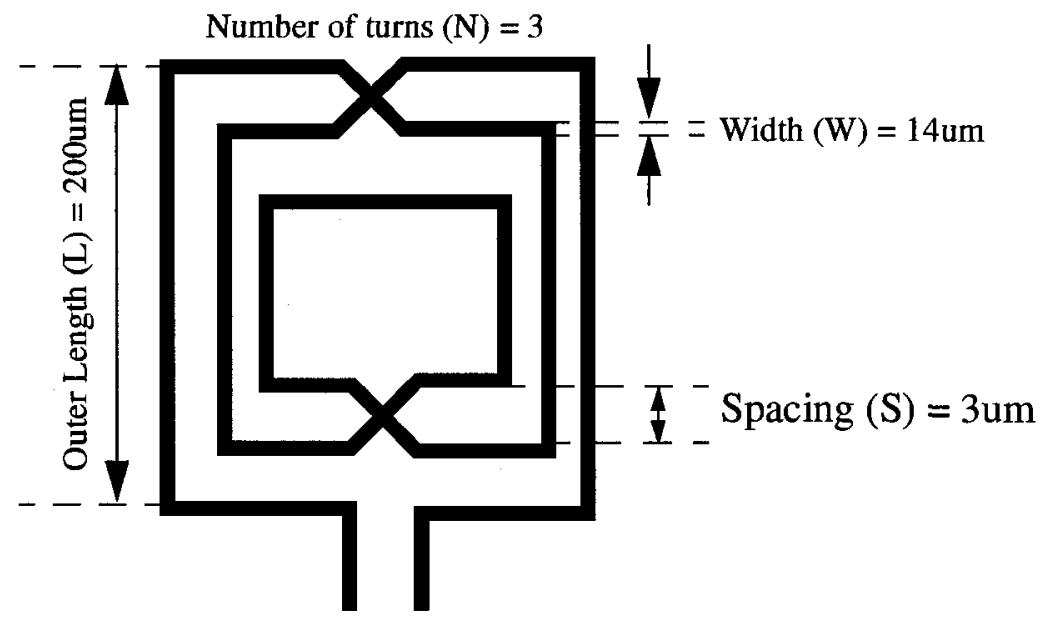

Figure 4.1: On-chip differential spiral inductor layout (not to scale) 
The outer length (L), the line width (W), the line spacing between metal lines (S), and the total number of turns $(\mathrm{N})$ defines the overall area of the on-chip spiral inductor. The differential routing requires a smaller area than two single-ended spiral inductor joined in series; and still provides the same required inductance value for our complementary "-gm" gain stage. Since the actual spiral inductor layout is covered by the foundry's inductor-recognition layer "IND", an illustrated example of the actual implemented spiral inductor is included in Figure 4.1 instead (see above).

\subsubsection{PN-junction Varactor (lateral PNP bipolar transistor)}

The PN-junction varactor is formed by lateral PNP bipolar transistor, which consists of 3 regions; namely emitter $(\mathrm{E})$, base $(\mathrm{B})$, and collector $(\mathrm{C})$. The emitter region is formed by $\mathrm{P}^{+}$materal (the same material as the drain/source region of a PMOS transistor). The base region is formed by $\mathrm{N}$ materal (the same material as the NWELL region of a PMOS transistor setup). The collector region is formed by the $\mathrm{P}^{+}$substrate, which is shared by all NMOS transistors in this technology. 
The dimension of the PN-junction varactor is defined by the lateral PNP bipolar transistor parametric-cell (or simply p-cell); which is supplied by the TSMC $0.18 \mathrm{um}$ library. In the passive-inductor-capacitor-VCO design, 8 such lateral PNP p-cells are connected in parallel. Each bipolar transistor is catergorized with a "PNP10" device model, which is used for simulation and also corresponds to the area of the emitter region in the device layout (see Figure 4.2).

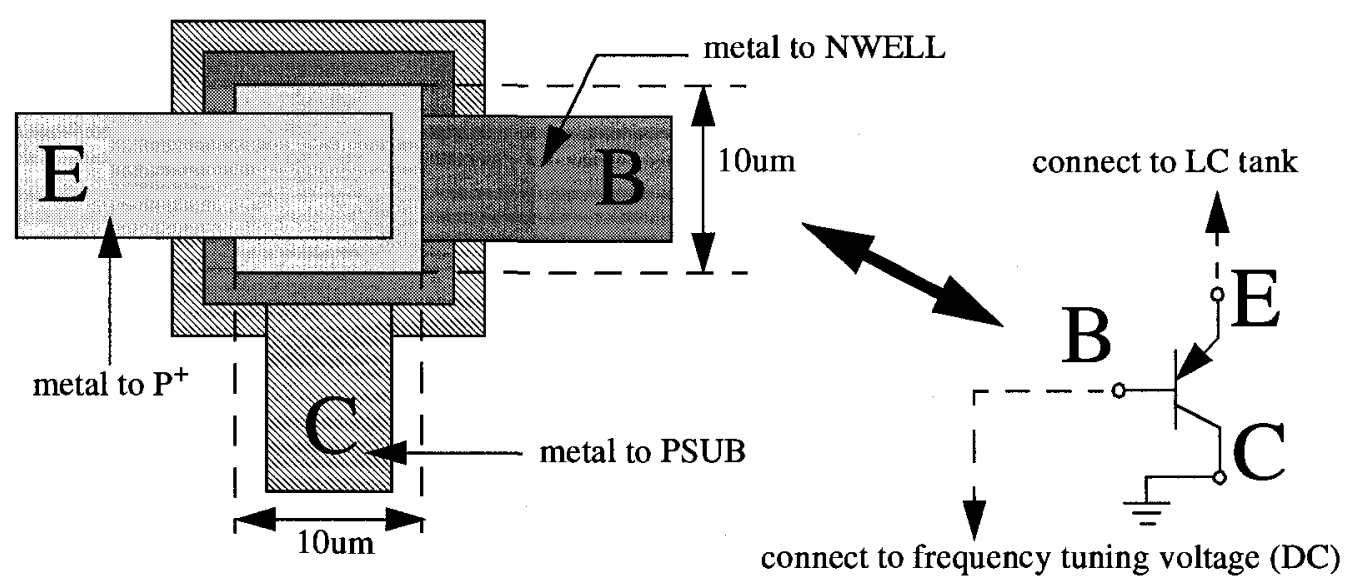

Figure 4.2: Layout of lateral PNP bipolar transistor as varactor (not to scale) 


\subsubsection{Complementary Negative-gm Gain Stage}

The complementary negative-gm gain stage consists of both PMOS and NMOS negative-gm gain stages. Each negative-gm gain stage is formed by a pair of MOS device (of same type, either $\mathrm{P}$ or $\mathrm{N}$ ) connected in the positive feedback manner. By connecting both PMOS and NMOS parts together, the complementary negative-gm gain stage resembles a single-ended CMOS latch (i.e. two CMOS inverter connected in the positive feedback configuration). In the current design, the PMOS and NMOS devices are sized as:

(PMOS negative-gm gain stage) $\quad \mathrm{W} / \mathrm{L} / \mathrm{m}=2.5 \mathrm{um} / 0.18 \mathrm{um} / 16 * 2$

(NMOS negative-gm gain stage) $\quad \mathrm{W} / \mathrm{L} / \mathrm{m}=2.5 \mathrm{um} / 0.18 \mathrm{um} / 16^{*} 2$

(NMOS input current mirror) $\quad \mathrm{W} / \mathrm{L} / \mathrm{m}=5 \mathrm{um} / 0.4 \mathrm{um} / 4$

(NMOS output current mirror) $\quad \mathrm{W} / \mathrm{L} / \mathrm{m}=5 \mathrm{um} / 0.4 \mathrm{um} / 20$ 


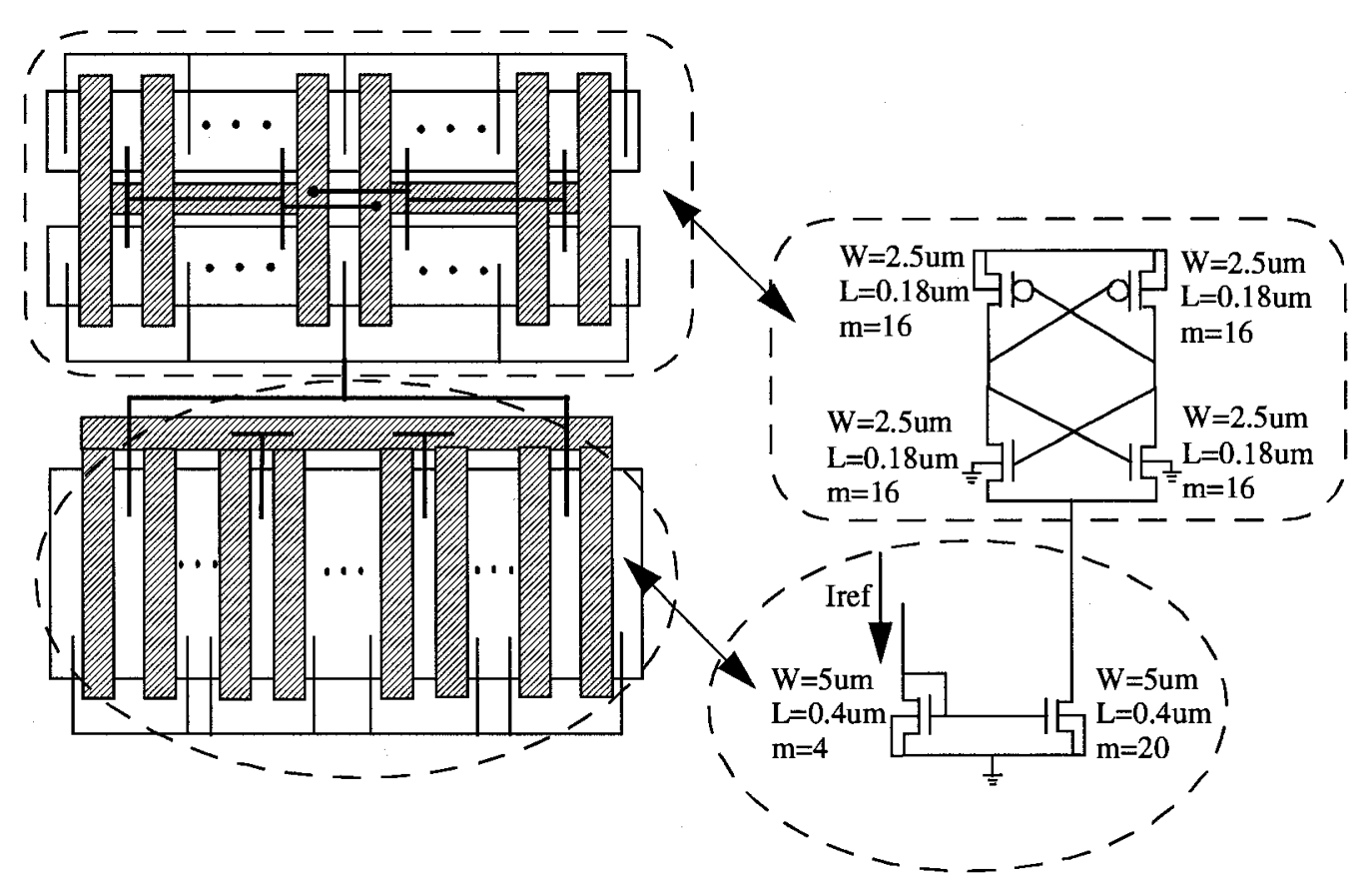

Figure 4.3: Layout of complementary negative-gm gain stage (not to scale)

As illustrated in Figure 4.3, the negative-gm gain stage is placed and routed as if it is a CMOS latch (i.e. 2 inverters connected in feedback). In addition, the negative feedback are routed in the centre of the latch structure to minimize parasitic capacitance due to routing. The biasing stage is a NMOS current mirror with a ratio of 1:5, "inter-digitizatioin" and "common-centroid" placement and routing techniques are employed to match the two sides of the current mirror as close to each other as possible. 


\subsubsection{Passive LC-VCO Buffer Stage}

The output buffer stage of the passive LC-VCO consists of 3 cascading gain stages. The first two stages are resistively-loaded “differential-pair" stages, whereas the last stage splits the two output signals by two single-ended "source-follower" stage. In addition, the buffer stage was designed for quadrature oscillator; hence only 1 of 2 channels was used for the passive LC-VCO circuit. The device dimensions are summarized as follows:

Reference: (NMOS input current mirror) $\quad \mathrm{W} / \mathrm{L} / \mathrm{m}=5 \mathrm{um} / 0.4 \mathrm{um} / 4$

1st stage: (NMOS output current mirror) $\quad \mathrm{W} / \mathrm{L} / \mathrm{m}=5 \mathrm{um} / 0.4 \mathrm{um} / 4$

1st stage: (NMOS differential pair, each device) $\mathrm{W} / \mathrm{L} / \mathrm{m}=1.5 \mathrm{um} / 0.18 \mathrm{um} / 4$

1st stage: $($ Rload $=811.304+405.652) \quad$ W/L/m=0.92um/2.4um/1+(1//1)

2nd stage: (NMOS output current mirror) $\quad \mathrm{W} / \mathrm{L} / \mathrm{m}=5 \mathrm{um} / 0.4 \mathrm{um} / 8$

2nd stage: (NMOS differential pair, each device)W/L/m=1.5um/0.18um/8

2nd stage: $($ Rload $=405.652)$

$\mathrm{W} / \mathrm{L} / \mathrm{m}=0.92 \mathrm{um} / 2.4 \mathrm{um} /(1 / / 1)$

3rd stage: (NMOS output current mirror) $\quad \mathrm{W} / \mathrm{L} / \mathrm{m}=5 \mathrm{um} / 0.4 \mathrm{um} / 12$

3rd stage: (NMOS source follower) $\quad \mathrm{W} / \mathrm{L} / \mathrm{m}=2.5 \mathrm{um} / 0.18 \mathrm{um} / 32$ 
Ideally the current mirror structure of the buffer stage should be placed and routed with "common centroid" and "interdigitization" techniques, but such placement requires a bigger overall area (i.e. for signal routing) and results in a larger parasitic capacitance. Hence the current mirrors circuit is designed to have identical unit finger width and length; and relies on compact placement for better matching (see Figure 4.4). Finally, dummy devices are placed next to the poly resistors for matching. 


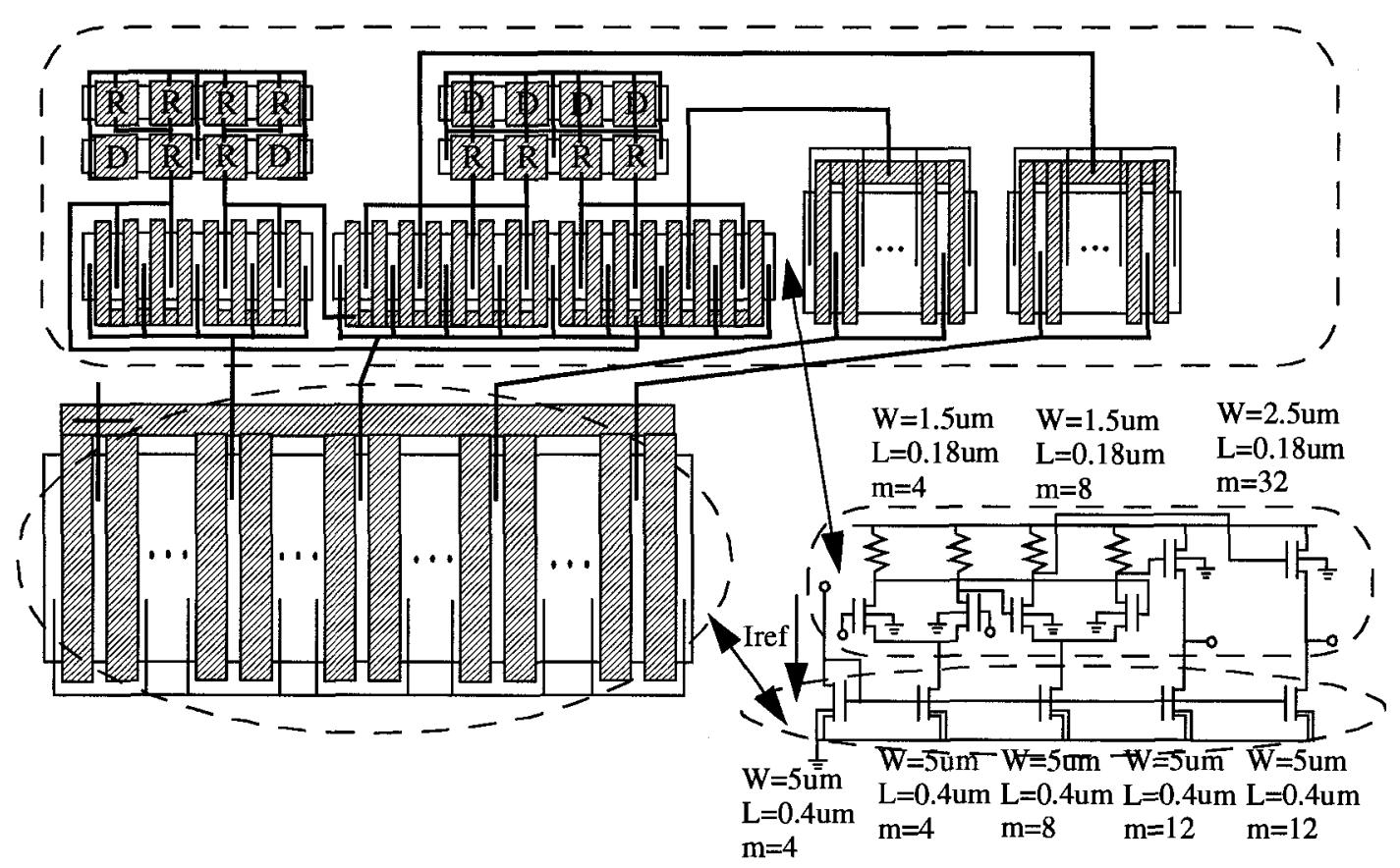

Figure 4.4: Layout of buffer for passive LC-VCO circuit (not to scale)

\subsubsection{Passive LC-VCO Circuit Layout (Top Level)}

Due to the limited die area granted for this research, the buffer circuit cannot be placed with same transistor orientation as other oscillator circuits (see Figure 4.5 and Figure 4.8). Tradeoff in this issue will be covered in section 4.3.

The top level passive LC-VCO circuit layout is shown as follows: 
3-stage output buffer (with mismatched orientation)

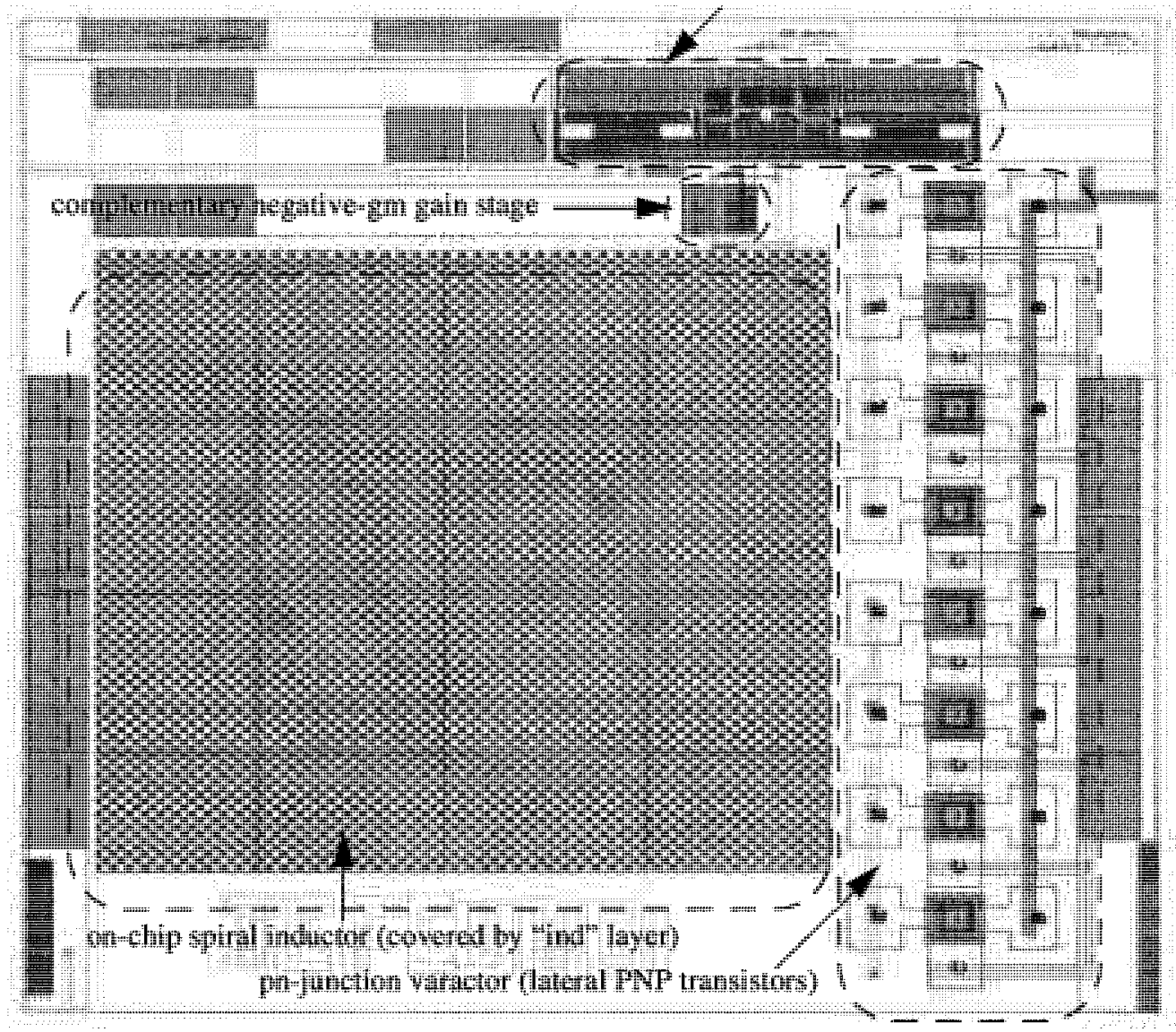

Figure 4.5: Layout of passive LC-VCO circuit (top level, not to scale) 


\subsection{Active-Inductor-Capacitor VCO Circuit Layout}

As described in Chapter 3, the active inductor-capacitor oscillator core circuit consists of three main components:

1. NMOS current mirror (for current bias of subsequent stages)

2. voltage-to-current-converter circuit (for oscillation frequency tuning)

3. 2-stage current-controlled-oscillator circuit (connected in direct-and-cross-coupled feedback manner, for quadrature oscillation output)

Since we have to drive the signal off-chip, there is a buffer circuit added in between the current controlled oscillator circuit and the wirebond pads.

\subsubsection{NMOS Current Mirror Circuit Layout}

The NMOS current mirror circuit is responsible for current biasing of both voltage-to-current converter and current-controlled-oscillator (CCO) circuits. The dimension of these devices are summarized as follows: 


\begin{tabular}{|c|c|}
\hline Reference: (NMOS input current mirror) & $\mathrm{W} / \mathrm{L} / \mathrm{m}=5 \mathrm{um} / 0.4 \mathrm{um} / 4$ \\
\hline 1st output: (to voltage-current converter) & $\mathrm{W} / \mathrm{L} / \mathrm{m}=5 \mathrm{um} / 0.4 \mathrm{um} / 2$ \\
\hline 2nd output:(to 1st stage CCO PMOS) & $\mathrm{W} / \mathrm{L} / \mathrm{m}=5 \mathrm{um} / 0.4 \mathrm{um} / 6$ \\
\hline 3rd output:(to 1 st stage CCO diff-pair) & $\mathrm{W} / \mathrm{L} / \mathrm{m}=5 \mathrm{um} / 0.4 \mathrm{um} / 8$ \\
\hline 4th output: (to 1st stage CCO feedback) & $\mathrm{W} / \mathrm{L} / \mathrm{m}=5 \mathrm{um} / 0.4 \mathrm{um} / 2$ \\
\hline 5th output: (to 2nd stage CCO PMOS) & $\mathrm{W} / \mathrm{L} / \mathrm{m}=5 \mathrm{um} / 0.4 \mathrm{um} / 6$ \\
\hline 6th output: (to 2nd stage CCO diff-pair) & $\mathrm{W} / \mathrm{L} / \mathrm{m}=5 \mathrm{um} / 0.4 \mathrm{um} / 8$ \\
\hline 7th output: (to 2 nd stage $\mathrm{CCO}$ fe & $\mathrm{W} / \mathrm{L} / \mathrm{m}=5 \mathrm{um} / 0.4 \mathrm{um} / 2$ \\
\hline
\end{tabular}

Since the primary goal of the oscillator circuit layout is to minimize the parasitic capacitance resulting from routing, placement of current mirror devices are determined by the location of their corresponding converter/oscillator circuits (i.e. signal path) instead of an "inter-digitization" layout technique. To compensate for above non-ideal layout choice, current mirror NMOS devices were designed with the same unit finger dimension and placed in close proximity; hence current sinks between input and output branches of the NMOS current mirror should have very close values. 


\subsubsection{Voltage-to-Current Converter Circuit Layout}

The voltage-to-current converter circuit consists of a PMOS differential pair with diode-connected NMOS devices as loads. The dimension of these devices are summarized as follows...

\begin{tabular}{|c|c|c|}
\hline Refere & (PMOS input current mirror) & $\mathrm{W} / \mathrm{L} / \mathrm{m}=5 \mathrm{um} / 0.4 \mathrm{um} / 8$ \\
\hline & (PMOS output current mirror) & $\mathrm{W} / \mathrm{L} / \mathrm{m}=5 \mathrm{um} / 0.4 \mathrm{um} / 4$ \\
\hline 2nd ou & :(PMOS output current mirror) & $\mathrm{W} / \mathrm{L} / \mathrm{m}=5 \mathrm{um} / 0.4 \mathrm{um} / 4$ \\
\hline input: & (PMOS diff-pair) & $\mathrm{W} / \mathrm{L} / \mathrm{m}=2.5 \mathrm{um} / 0.2 \mathrm{um} / 8$ \\
\hline degen & (PMOS diff-pair degeneration) & $\mathrm{W} / \mathrm{L} / \mathrm{m}=2.5 \mathrm{um} / 0.2 \mathrm{um} / 2$ \\
\hline oad: & (diode-connected NMOS load) & 5um/0.4um/2 \\
\hline
\end{tabular}

In order to provide better matching among the PMOS devices among both differential pair and degenerative devices, all PMOS devices were designed with the same unit finger width and length. In addition, the degenerative PMOS devices are placed at the centre of the differential pair circuit for symmetrical layout. 
Similarly, PMOS current mirrors are placed and routed with "common centroid" and interdigitization" layout techniques, for better matching among input and 2 output current mirror branches.

Finally, the diode-connected NMOS loads are actually input current mirror branches of the current-controlled-oscillator frequency-tuning current mirror. Hence they are placed close to the output current mirror branches (i.e. part of the current-controlledoscillator frequency-tuning circuit); and were designed to have the same unit finger width and length.

\subsubsection{Current-controlled-oscillator Circuit Layout}

The current-controlled-oscillator circuit consists of two differential gain stages, connected in direct and crossed configuration. This connection is part of the active inductance generation circuit. The dimension of these devices are summarized as follows:

PMOS input current mirror:(1st stage bias) $\mathrm{W} / \mathrm{L} / \mathrm{m}=1.25 \mathrm{um} / 0.2 \mathrm{um} / 12$

PMOS output current mirror:(1st stage load) $\mathrm{W} / \mathrm{L} / \mathrm{m}=1.25 \mathrm{um} / 0.2 \mathrm{um} / 12$

NMOS differential pair: (1st stage) $\quad \mathrm{W} / \mathrm{L} / \mathrm{m}=1.25 \mathrm{um} / 0.18 \mathrm{um} / 12$ 
NMOS positive feedback:(1st stage) W/L/m=1.25um/0.18um $/ 4$

PMOS input current mirror:(2nd stage bias) $\mathrm{W} / \mathrm{L} / \mathrm{m}=1.25 \mathrm{um} / 0.2 \mathrm{um} / 12$

PMOS output current mirror:(2nd stage load)W/L/m=1.25um/0.2um $/ 12$

NMOS differential pair: (2nd stage) $\quad \mathrm{W} / \mathrm{L} / \mathrm{m}=1.25 \mathrm{um} / 0.18 \mathrm{um} / 12$

NMOS positive feedback:(2nd stage) $\quad$ W/L/m=1.25um/0.18um/4

For minimal parasitic capacitance, the two differential gain stages are placed sideby-side.

\subsubsection{Active LC-VCO Buffer Stage}

The output buffer stage of the active LC-VCO consists of 3 cascading gain stages. It is used for both active and passive LC-VCO circuits. The device dimensions are summarized as follows:

Reference: (NMOS input current mirror) W/L/m=5um/0.4um/4

1st stage: (NMOS output current mirror) $\quad \mathrm{W} / \mathrm{L} / \mathrm{m}=5 \mathrm{um} / 0.4 \mathrm{um} / 4$

1st stage: (NMOS differential pair, each device)W/L/m=1.5um/0.18um/4

1st stage: $(\operatorname{Rload}=811.304+405.652) \quad \mathrm{W} / \mathrm{L} / \mathrm{m}=0.92 \mathrm{um} / 2.4 \mathrm{um} / 1+(1 / / 1)$

2nd stage: (NMOS output current mirror) $\quad \mathrm{W} / \mathrm{L} / \mathrm{m}=5 \mathrm{um} / 0.4 \mathrm{um} / 8$ 
2nd stage: (NMOS differential pair, each device) $\mathrm{W} / \mathrm{L} / \mathrm{m}=1.5 \mathrm{um} / 0.18 \mathrm{um} / 8$

2nd stage: $($ Rload $=405.652) \quad \mathrm{W} / \mathrm{L} / \mathrm{m}=0.92 \mathrm{um} / 2.4 \mathrm{um} /(1 / / 1)$

3rd stage: (NMOS output current mirror) $\quad \mathrm{W} / \mathrm{L} / \mathrm{m}=5 \mathrm{um} / 0.4 \mathrm{um} / 12$

3rd stage: (NMOS source follower) $\quad \mathrm{W} / \mathrm{L} / \mathrm{m}=2.5 \mathrm{um} / 0.18 \mathrm{um} / 32$

\subsubsection{Active LC-VCO Circuits Layout}

Since the active LC-VCO relies on the transformation from parasitic capacitance to active inductance, parasitic capacitance must be minimized for a high oscillation frequency.

From the component level of the oscillator circuit layout, differential pair and current mirror circuits are placed in close proximities for both performance matching and routing parasitic minimization.

From the top level of the oscillator circuit layout, output buffer circuits (i.e. for both active and passive LC-VCOs) are placed close to the wire-bond "landing" pads. Similarly, the active LC-VCO circuit is placed close to its corresponding buffer circuit. 


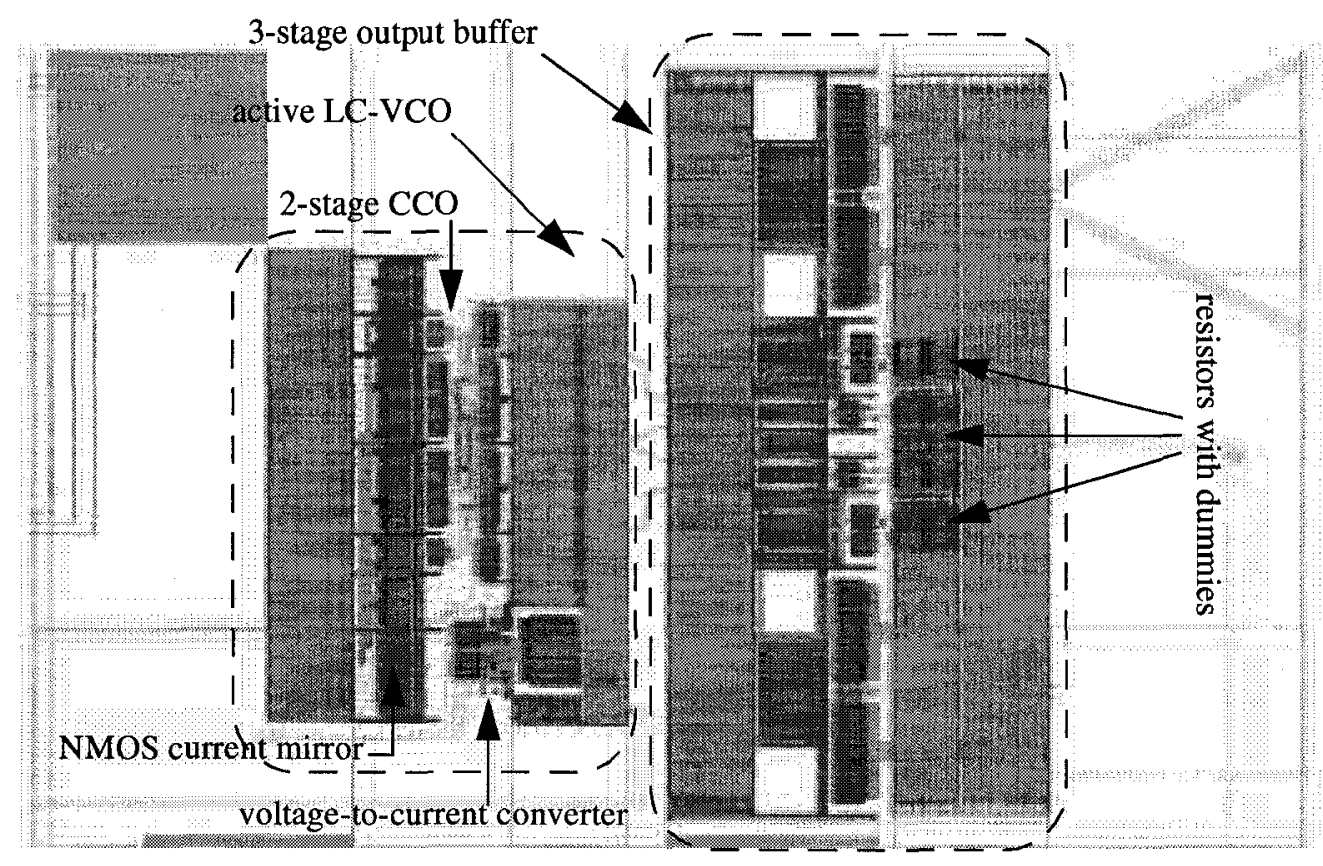

Figure 4.6: Layout of active LC-VCO circuit (not to scale)

\subsection{Top Level Circuit Layout}

This section illustrates the layout of the test chip, which incorporates both active and passive LC-VCO circuits. Two options of chip-board interconnection media were offered by CMC: "flip-chip" and "wire-bonding" 
The first option, called "flip-chip", is a newer technology. This type of interconnection connects input and output pads (or simply "I/O pads", consisting various top metal layers) directly from the test chip onto the corresponding chip package routing lines. Hence the chip is "flipped" upside-down for such interconnection, and the technology is generally named after this technique. The advantages of such an interconnection includes support of higher speed signal routing (which is important for high speed wireline and wireless circuits and systems) and flexibility of signal connections within the entire test chip area. The disadvantage of this technology is its relatively high cost.

The second option is an older technology called "wire-bonding". This type of interconnection connects $\mathrm{I} / \mathrm{O}$ pads from test chip onto the chip package routing lines through a wire. The advantage of such interconnection is its relatively cheaper cost; wherease disadvantages include the limitation on I/O pads placement locations, and the low speed signal propagations along "bond-wires".

The "wire-bonding" interconnection technology is chosen for this test chip, hence all I/O pads are placed along the perimeter of the test chip. Although the total area of the three oscillator circuits is much smaller than the granted die area of $1 \mathrm{~mm}^{2}$, the test chip is limited by the I/O pad layout area. In other words, this test chip is "pad-limited"; and the 
number of I/O pads can only support three versions of the oscillator circuit. Moreover, the differential input signals are shared among the two versions of active LC-VCO circuits (i.e. one of them is a backup for test purpose). Finally, only one of two differential output signal pairs (from each active LC-VCO circuit) is wire-bonded for testing.

Due to the limited die area for this test chip (i.e. "pad-limited"), placement of the passive LC-VCO buffer circuit is orthongonal to the active LC-VCO circuit, which is incorrect for MOS device performance tracking. In this way, the only performance mismatch effect (if any, due to fabrication variation on device dimensions) will be on the current mirrors of the buffer circuits; but not in the oscillator circuits themselves.

The test chip layout is included as follows: 


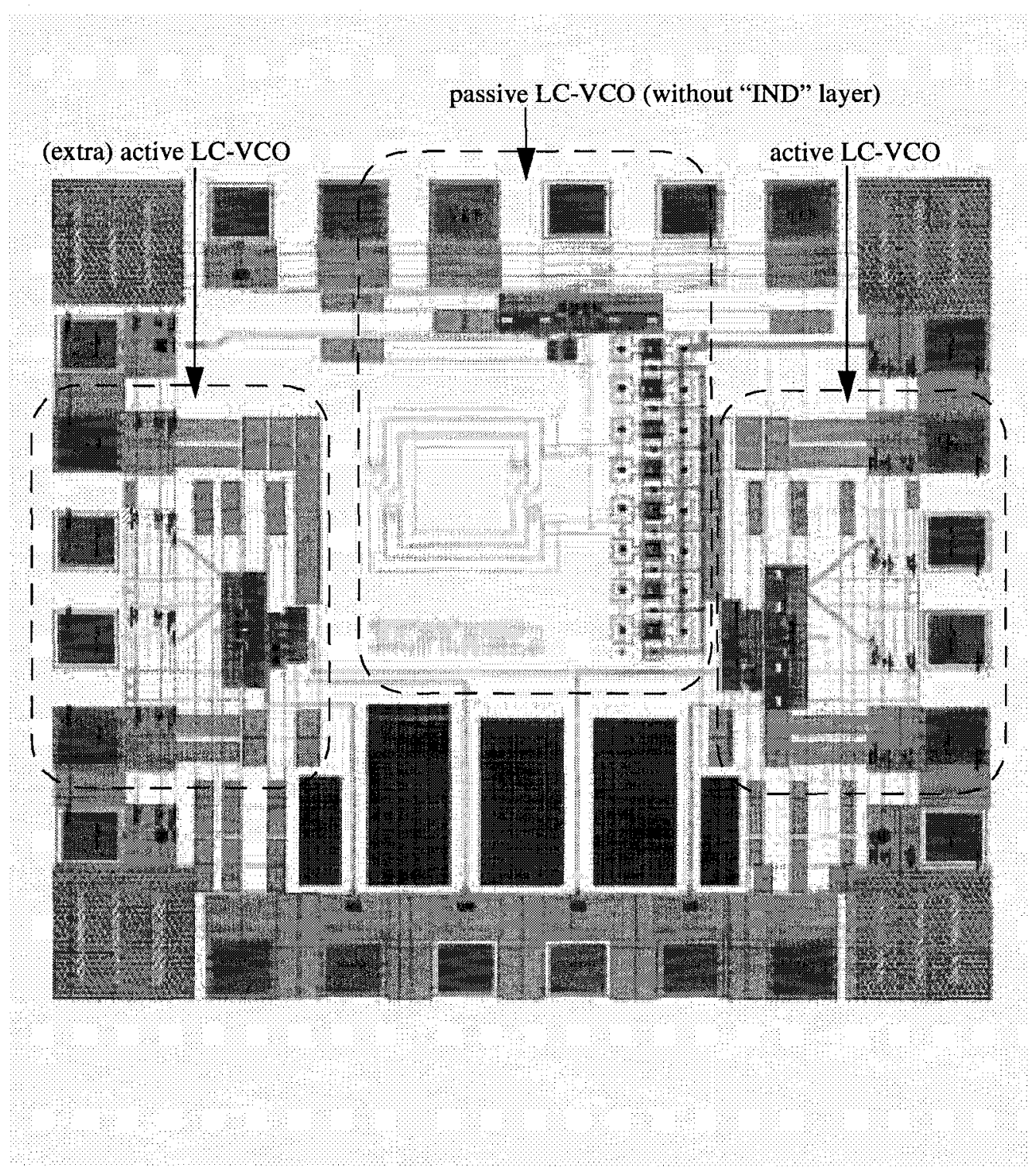

Figure 4.7: Layout of active LC-VCO circuit (2nd version, not to scale) 


\subsection{Off-Chip Circuit Test Board}

Since the output frequencies of both active and passive LC-VCO circuits are close to $5 \mathrm{GHz}$, chip package selections provided by CMC Microsystems cannot be used for this research (i.e. CMC Microsystems chip packages can support signals up to $2 \mathrm{GHz}$ only).

With help from Dr. Tarr and his research team, a test board is fabricated to meet this strict requirement. The structure of this test board consists of a 1um-thick gold layer doped onto a 2"x2" glass plate. Multiple test sites are prepared on this 2"x2" test structure (see Figure 4.8).

Detail information on the wirebonding for both active and passive LC-VCO circuits are illustrated in next sub-sections. 


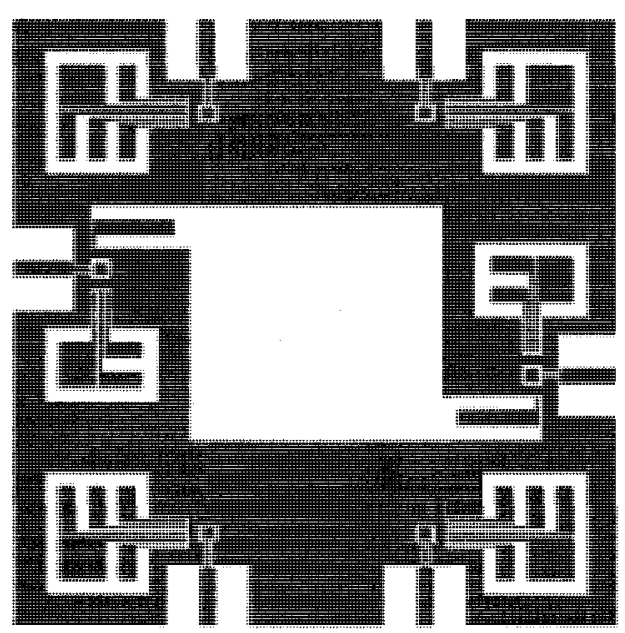

Figure 4.8: gold plated 2'x2" test structure on glass (to scale)

\subsubsection{Active LC-VCO Test Site And Wirebonding Diagram}

Since chip packaging is omitted for this research, bond wires are placed between the on-chip input/output (or I/O) pads and the off-chip wirebond sites. As oscillation frequencies are varied by differential tuning voltages, narrow-band impedance matching for the buffer circuit output nodes are omitted; and a short gold trace is placed between the off-chip wirebonding site and the SMA connector site (see Figure 4.9). 


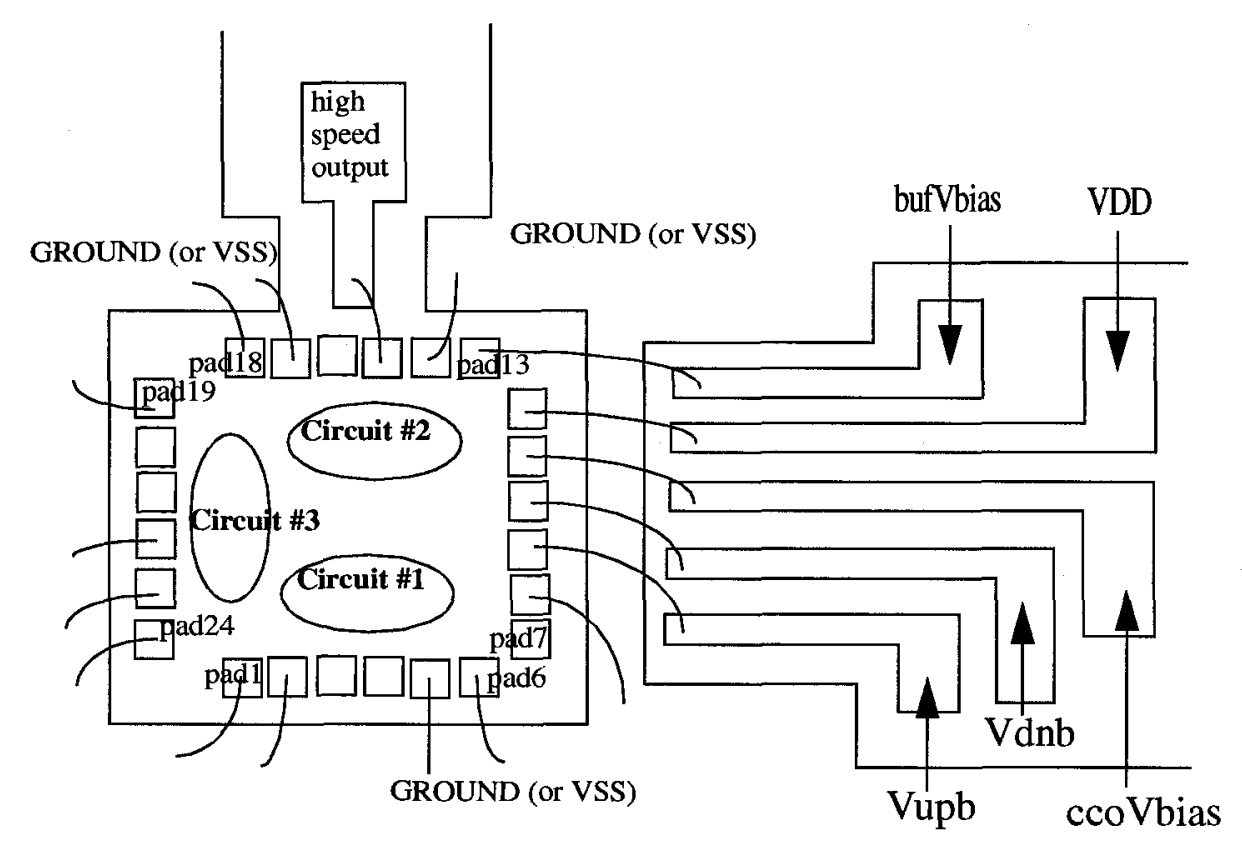

Figure 4.9: Active LC-VCO test site and wirebonding diagram

Since "Circuit\#2" is the designated device under test (or DUT), control voltages to the other two circuits are wirebonded to ground. In addition, bias and control pins to the active LC-VCO circuit are summarized as follows:

- "Vupb" and "Vdnb" are diferential input pins of the voltage-to-current converter circuit

- "ccoVbias" and "bufVbias" are input pins to the NMOS current mirrors of both current controlled oscillator and buffer circuits respectively 


\subsubsection{Passive LC-VCO Test Site And Wirebonding Diagram}

Similar to the active LC-VCO test setup, narrow-band impedance matching for the passive LC-VCO buffer circuit output nodes are omitted; and a short gold trace is placed between the off-chip wire-bonding site and the SMA connector site (see Figure 4.10).

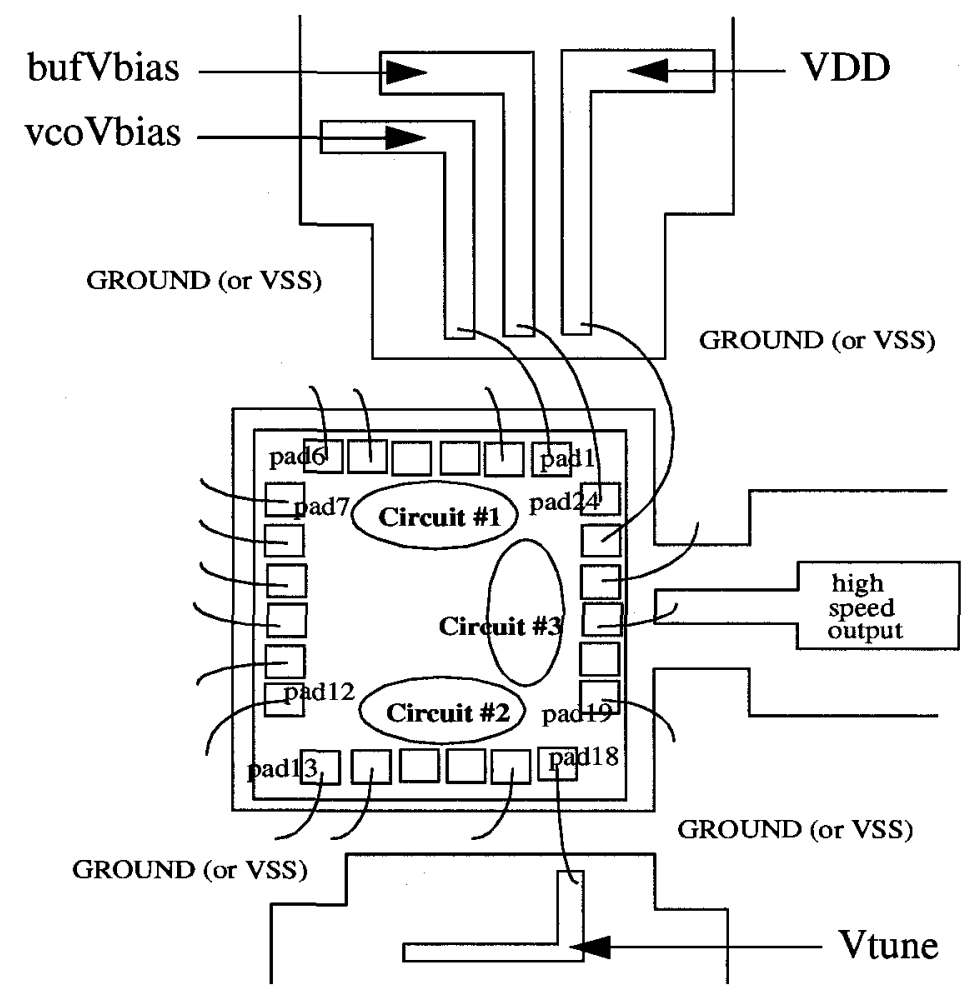

Figure 4.10: Passive LC-VCO test site and wirebonding diagram 
Since "Circuit\#3" is the designated device under test (or DUT), control voltages to the other two circuits are wirebonded to ground. In addition, bias and control pins to the passive LC-VCO circuit are summarized as follows:

- "Vtune" is connected to the "base" terminal of the 8 shunt-connected PNP varactors

- "vcoVbias" and "bufVbias" are input pins to the NMOS current mirrors of both LCVCO and buffer circuits respectively

\subsection{Lab Test Equipment}

This section covers circuit test setup and related test equipment. The lab measurements of both active and passive LC-VCO circuits are included in Chapter 5, and the list of lab test equipment is included as follows:

- Tektronix TDS6604 Digital Storage Oscilloscope (1 unit)

- Agilent E4407B Spectrum Analyzer (1 unit)

- Agilent E3646 Dual Power Supply (3 units) 
A picture is taken during the test of both active and passive LC-VCO cirucits:

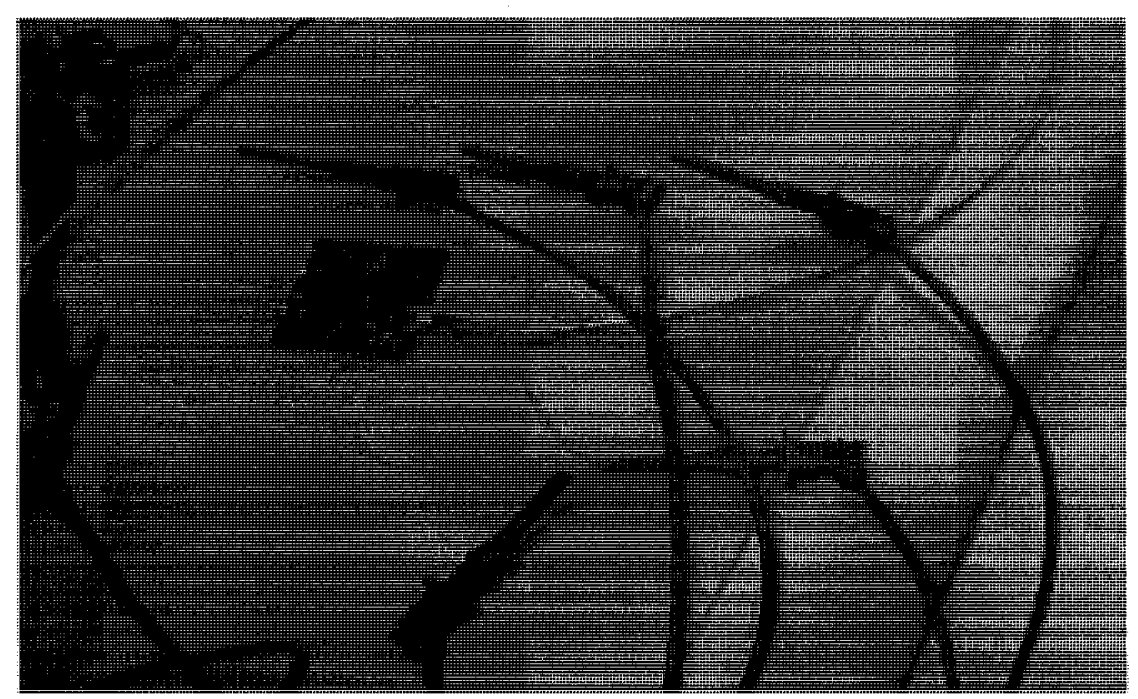

Figure 4.11: oscillators under tests

\subsection{Circuit Layout Summary}

One passive and two active LC-VCO circuit layout have been included on the testchip. Due to the pad-limited configuration, differential input pins for both versions of active LC-VCO circuits are shared. On testboard level, six test configurations are fabricated on a 1um thick gold plated glass substrate. Within each test configuration, a singleended output and a few input and power pads have been wirebonded for test purpose. Comparisons and discussions on simulated and measured results of both oscillator circuits will be covered in next chapter. 
This chapter covers the simulation and measured results of both passive and active LC-VCO circuits, and is divided into three sub-sections.

The first part of this chapter provides background information on various existing phase noise models, with equations reproduced from various phase noise papers. The second part of this chapter summarizes both simulation and measured results of the active LC-VCO circuit; and provide insights on root-cause and solutions for sub-optimal measured results from the circuit. The third part of this chapter focus on the passive LC-VCO circuit's simulation and measured results. In addition, discussions on root-cause and solu- 
tions for issues related to the passive LC-VCO circuit will be covered at the end of this chapter.

\subsection{Phase noise and existing models}

Phase noise is an indicator of an oscillator circuit's performance; and is defined as a ratio between the single side-band power at an offset frequency from the carrier (with a 1 $\mathrm{Hz}$ bandwidth) and the carrier signal power (see Equation 5.1) [5].

$$
L\{\Delta \omega\}=10 \cdot \log \left(\frac{P_{\text {sideband }}\left(\omega_{o}+\Delta \omega, 1 H z\right)}{P_{\text {carrier }}}\right)
$$

where " $\mathrm{L}\{\Delta \omega\}$ " is the phase noise, " $\mathrm{P}_{\text {sideband" is the signal power at the offset fre- }}$ quency, " $\mathrm{P}_{\text {carrier }}$ " is the carrier signal power, " $\omega_{0}$ " is the carrier frequency, and " $\Delta \omega$ " is the offset frequency away from the carrier.

The spectrum of an oscillator output signal can be categorized into three regions, namely the $1 / f^{3}, 1 / f^{2}$, and a flat region. The $1 / f^{3}$ region is caused by the MOS devices' flicker noise, which has a $1 / \mathrm{f}$ spectral profile. The $1 / \mathrm{f}^{2}$ region is caused by the white noise 
inside the oscillator circuit, which has a constant value over the frequency spectrum.

Finally, the flat region in the oscillator output spectrum is caused by the white noise in the oscillator buffer circuit; which is located outside the oscillator feedback loop. Hence the "flat" spectral profile of the white noise remains unchanged.

\subsubsection{Leeson's phase noise model}

In 1966, Leeson proposed a phase noise model that reveals the relationship between the oscillator input phase uncertainty and the power spectral density of both phase and frequency of signals at the oscillator circuit output [1]. This model is summarized as:

$$
S_{\varnothing}\left(\omega_{m}\right)=S_{\Delta \theta} \cdot\left[1+\left(\frac{\omega_{o}}{2 \cdot Q \cdot \omega_{m}}\right)^{2}\right]
$$

where

" $\mathrm{S}_{\phi}\left(\omega_{\mathrm{m}}\right)$ " is the power spectral density of the oscillator circuit output frequency

" $\mathrm{S}_{\Delta \theta}$ " is the oscillator circuit input phase uncertainty

" $\omega_{0}$ " is the output oscillation frequency 
"Q" is the loaded quality factor of the oscillator circuit

" $\omega_{\mathrm{m}}$ " is the offset frequency in the oscillator output frequency spectrum

In addition, the relationship between the oscillator input phase uncertainty and oscillator output phase/frequency power spectra is illustrated as follows:

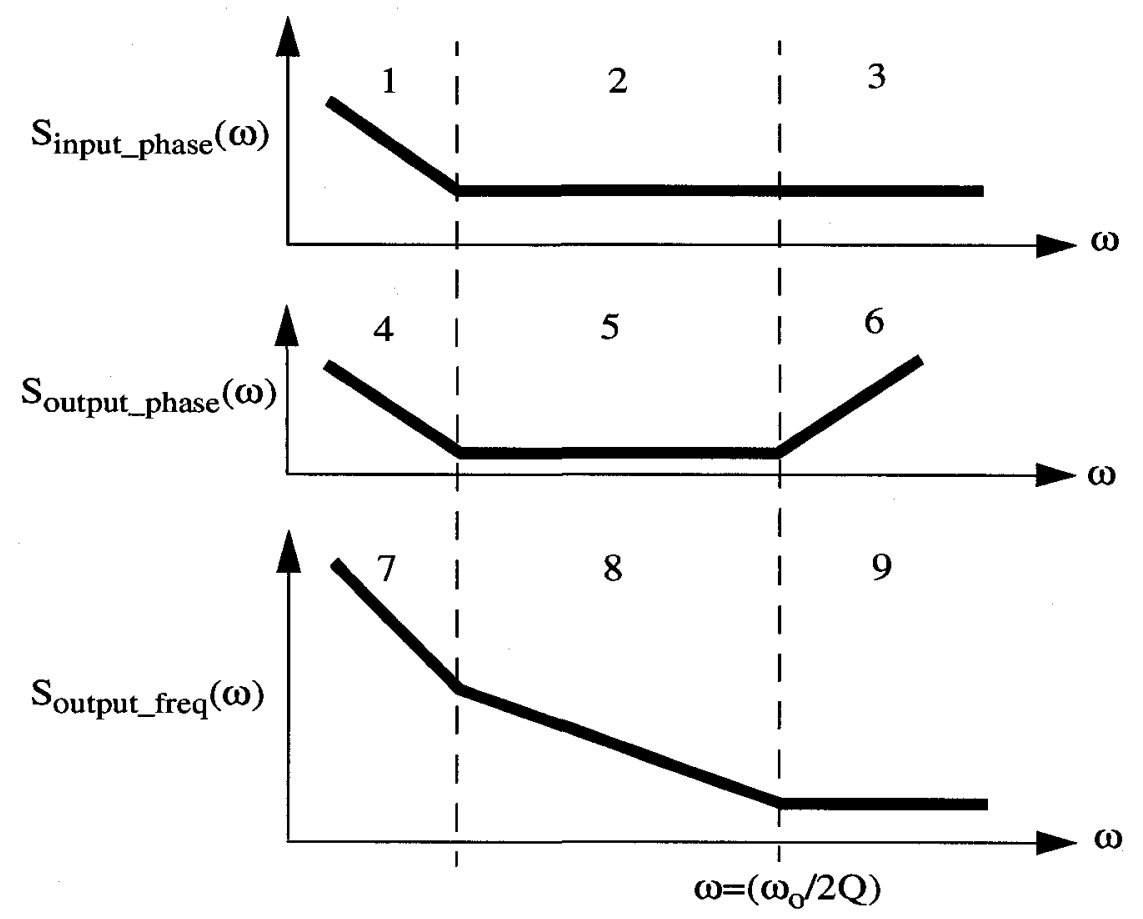

Figure 5.1: Power Spectral Densities at oscillator input and output

In region 1 , the input phase is: 


$$
S_{\Delta \theta}(\omega)=\alpha \cdot\left(\frac{1}{\omega_{m}}\right)
$$

At the oscillator input, phase power spectral density decreases at a rate of $20 \mathrm{~dB}$ / decade. This decreases continue until the device flicker noise (or $1 / \mathrm{f}$ noise) effect no long dominates the overall noise. In addition, the " $\alpha$ " variable in equations 5.2 to 5.9 is a constant used to represents the $1 / \mathrm{f}$ noise effect.

In regions 2 and 3 , the input phase is:

$$
S_{\Delta \theta}(\omega)=\frac{2 \cdot F \cdot K \cdot T}{P_{s}}
$$

In region 4 , the output phase is:

$$
S_{\varnothing^{\circ}}(\omega)=\alpha \cdot\left(\frac{1}{\omega_{m}}\right) \cdot\left(\frac{\omega_{o}}{2 \cdot Q}\right)^{2}
$$

At the oscillator output, the phase spectrum decreases at a rate of $-20 \mathrm{~dB} / \mathrm{decade}$. This is almost identical to the oscillator input, except the value has been multiplied by a factor $\left(\omega_{\mathrm{o}} / 2 \mathrm{Q}\right)^{2}$. Hence, this effect continues (i.e. along the offset frequency scale) until the $1 / f$ noise no longer dominates the overall device noise. This factor depends on both the oscillation frequency " $\omega_{0}$ " and the loaded quality " $Q$ " of the oscillator circuit. 
In region 5, the output phase is:

$$
S_{\varnothing \circ}(\omega)=\left(\frac{2 \cdot F \cdot K \cdot T}{P_{s}}\right) \cdot\left(\frac{\omega_{o}}{2 \cdot Q}\right)^{2}
$$

At the oscillator output, the phase spectrum does not vary with the offset frequency. The value is almost identical to the oscillator input, except it has been multiplied by a factor of $\left(\omega_{0} / 2 Q\right)^{2}$. Once again, this factor depends on both the oscillation frequency " $\omega_{0}$ " and the loaded quality " $Q$ " of the oscillator circuit.

In region 6 , the output phase is:

$$
S_{\varnothing \circ}(\omega)=\left(\frac{2 \cdot F \cdot K \cdot T}{P_{s}}\right) \cdot\left(\omega_{m}\right)^{2}
$$

At the oscillator output, the phase spectrum increases at a rate of $+40 \mathrm{~dB} / \mathrm{dec}$ de. This value only depends on the offset frequency " $\omega_{m}$ ", but not with the oscillation frequency " $\omega_{0}$ " nor the loaded quality " $Q$ " of the oscillator circuit.

In region 7 , the output frequency is: 


$$
S_{\varnothing}(\omega)=\alpha \cdot\left(\frac{1}{\omega_{m}}\right)^{3} \cdot\left(\frac{\omega_{o}}{2 \cdot Q}\right)^{2}
$$

At the oscillator output, the frequency spectrum decreases at a rate of $-60 \mathrm{~dB} / \mathrm{dec}$.

Similar to regions 1 and 4, this noise profile is predominated by the device's $1 / \mathrm{f}$ noise.

In region 8 , the output frequency is:

$$
S_{\varnothing \circ}(\omega)=\left(\frac{2 \cdot F \cdot K \cdot T}{P_{s}}\right) \cdot\left(\frac{1}{\omega_{m}}\right)^{2} \cdot\left(\frac{\omega_{o}}{2 \cdot Q}\right)^{2}
$$

At the oscillator output, the frequency spectrum decreases at a rate of $-40 \mathrm{~dB} / \mathrm{dec}$. This corresponds to the relatively flat phase output spectrum of region 5 , but the profile in the frequency spectrum decreases further.

In region 9 , the output frequency is:

$$
S_{\Delta \theta}(\omega)=\frac{2 \cdot F \cdot K \cdot T}{P_{s}}
$$

At the oscillator output, the frequency spectrum has an identical profile as the phase spectrum at the oscillator input, which also corresponds to the oscillator output phase spectrum; but without the variation due to the offset frequency. The flat frequency 
spectrum is due to additive noise originated from oscillator buffer circuit, which is placed between the oscillator core circuit and I/O pads.

\subsubsection{Razavi's phase noise model}

Razavi has proposed the concept of an "open-loop" quality factor, which was used to describe a ring oscillator circuit's characteristics. It measures how a closed-loop circuit opposes variation in its oscillation frequency [4].

The "open-loop" $Q$ factor is defined as:

$$
Q=\frac{\omega_{o}}{2} \cdot \sqrt{\left(\frac{d A(\omega)}{d \omega}\right)^{2}+\left(\frac{d \varnothing(\omega)}{d \omega}\right)^{2}}
$$

where $A(\omega)$ and $\phi(\omega)$ are amplitude and phase functions of the "open-loop" ring oscillator circuit respectively.

In addition, the transfer function of this "open-loop" ring oscillator circuit is defined as: 


$$
H(j \omega)=A(\omega) \cdot e^{j \emptyset(t)} \approx H\left(j \omega_{o}\right)+\Delta \omega \cdot \frac{d H(\omega)}{d \omega}
$$

where

$$
\omega=\omega_{o}+\Delta \omega
$$

represents the frequency of interest, which includes an offset frequency of " $\Delta \omega$ " from the ideal clock frequency of " $\omega_{0}$ ".

Razavi has utilized a linear closed-loop oscillator model for his phase noise formula derivation (see Figure 5.2), the closed-loop system is summarized as:

$$
\frac{Y(j \omega)}{X(j \omega)}=\frac{H(j \omega)}{1+H(j \omega)}
$$

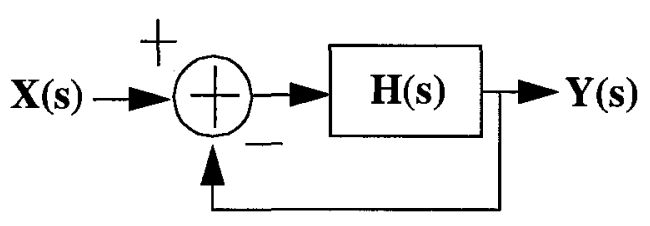

Figure 5.2: Razavi's linear closed-loop oscillator model 
Hence the noise transfer function at the frequency of interest is:

$$
\frac{Y\left(j \omega_{o}+j \Delta \omega\right)}{X\left(j \omega_{o}+j \Delta \omega\right)}=\frac{H\left(j \omega_{0}\right)+\Delta \omega \cdot \frac{d H(\omega)}{d \omega}}{1+H\left(j \omega_{o}\right)+\Delta \omega \cdot \frac{d H(\omega)}{d \omega}} \approx \frac{-1}{\Delta \omega \cdot \frac{d H(\omega)}{d \omega}}+1 \approx \frac{-1}{\Delta \omega \cdot \frac{d H(\omega)}{d \omega}}
$$

where

$$
H\left(j \omega_{o}\right)=-1
$$

and

$$
\left|\Delta \omega \cdot \frac{d H(\omega)}{d \omega}\right| \ll 1
$$

Equation 5.15 reveals a multiplicative effect from the closed-loop system onto all input noise, which also includes noise within the oscillator circuit.

Furthermore, the noise power spectral density is shaped (by the same closed-loop system) with this transformation:

$$
\left|\frac{Y\left(j \omega_{o}+j \Delta \omega\right)}{X\left(j \omega_{o}+j \Delta \omega\right)}\right|^{2}=\left|\frac{-1}{\Delta \omega \cdot \frac{d H(\omega)}{d \omega}}\right|^{2}=\frac{1}{(\Delta \omega)^{2} \cdot\left|\frac{d H(\omega)}{d \omega}\right|^{2}}
$$

The derivative of Equation 5.12 is: 


$$
\frac{d H(j \omega)}{d \omega}=\left(\frac{d A(\omega)}{d \omega}+j A \cdot \frac{d \varnothing(\omega)}{d \omega}\right) \cdot \exp (j \varnothing(\omega))
$$

Substitute Equation 5.19 into Equation 5.18:

$$
\left|\frac{Y\left(j \omega_{o}+j \Delta \omega\right)}{X\left(j \omega_{o}+j \Delta \omega\right)}\right|^{2}=\frac{1}{(\Delta \omega)^{2} \cdot\left|\frac{d H(\omega)}{d \omega}\right|^{2}}=\frac{1}{(\Delta \omega)^{2} \cdot\left[\left(\frac{d A(\omega)}{d \omega}\right)^{2}+\left(\frac{d \varnothing(\omega)}{d \omega}\right)^{2}\right]}
$$

where

$$
|A(\omega)|=1
$$

Substitute Equation 5.11 into Equation 5.20:

$$
\left|\frac{Y\left(j \omega_{o}+j \Delta \omega\right)}{X\left(j \omega_{o}+j \Delta \omega\right)}\right|^{2}=\frac{1}{(\Delta \omega)^{2} \cdot\left[\left(\frac{d A(\omega)}{d \omega}\right)^{2}+\left(\frac{d \varnothing(\omega)}{d \omega}\right)^{2}\right]}=\frac{1}{4 \cdot Q^{2}} \cdot\left(\frac{\omega_{o}}{\Delta \omega}\right)^{2}=\left(\frac{\omega_{o}}{2 \cdot Q}\right)^{2} \cdot\left(\frac{1}{\Delta \omega}\right)^{2}
$$

Hence Razavi's phase noise model depends on the oscillation frequency " $\omega_{0}$ ", the open-loop quality factor " $Q$ ", and the offset frequency " $\Delta \omega$ ". In addition, its profile decreases at a rate of $-40 \mathrm{~dB} / \mathrm{dec}$ ade along the offset frequency axis; which corresponds to region 8 of Leeson's phase noise model. 


\subsubsection{Hajimiri's phase noise model (for LC-VCO)}

Hajimiri has proposed a linear, time variant (or LTV) phase noise model; which explains the up-conversion mechanism of flicker noise and down-conversion of white noise into close-in phase noise at an oscillator output spectrum [5],[6],[10],[11],[13]. This sub-section illustrates his proposed phase noise model and its application to inductorcapacitor based oscillaor circuits; whereas the application to ring oscillator circuits will be revealed in the next sub-section.

Hajimiri introduced a periodic "impulse sensitivity function" (or ISF), which models after the sensitivity of an oscillator circuit output waveform to a current impulse applied to the circuit (at various time instances within its oscillation period). The effect from such an impulse varies a lot; and this variation depends heavily on the location of the output waveform at the time instance when the impulse is applied (see Figure 5.3). 


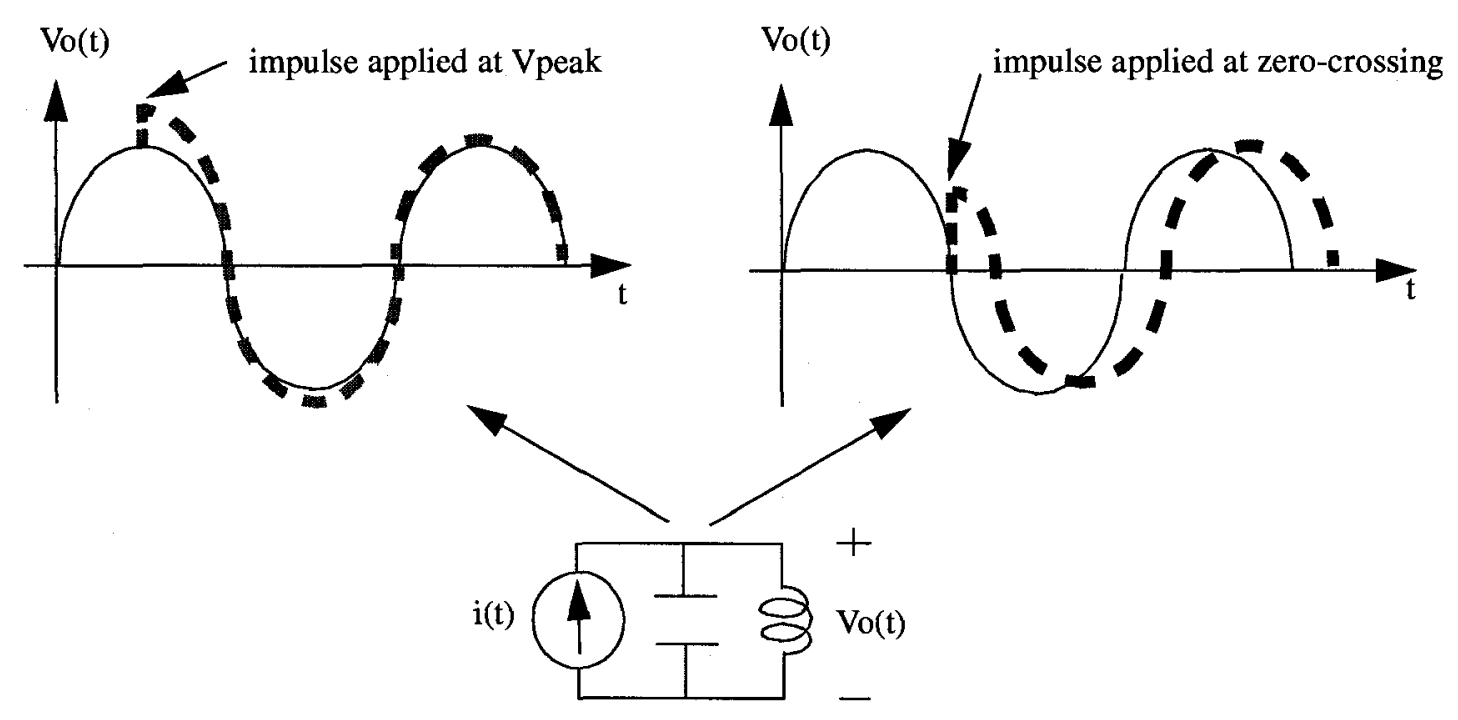

Figure 5.3: Impulse Sensitivity Function of an LC-VCO

If a current impulse is applied to the oscillator output node when the output voltage waveform is at its peak, then the amplitude of the waveform increases momentarily; but both the zero-crossing point and the period of the oscillation waveform remain unchanged (see curve in left hand side of Figure 5.3).

On the other hand, if the impulse is applied to the oscillator output node when the output waveform is at the zero-crossing point, then the period of the oscillation is changed; and there is no mechanism that can correct this disturbance (see curve in right 
hand side of Figure 5.3). In other words, the oscillator circuit is least/most sensitive to a current impulse disturbance when its output voltage waveform reaches the peak/zerocrossing locations respectively.

The oscillator output voltage waveform and an applied current impulse are represented (respectively) as follows:

$$
V_{o u t}(t)=A(t) \cdot f\left[\omega_{0} t+\phi(t)\right]
$$

and

$$
i(t)=I_{n} \cdot \cos \left[\left(n \omega_{0}+\Delta \omega\right) t\right]
$$

Since the oscillator output voltage waveform changes between peak and trough values once within each period, the impulse sensitivity function (or ISF) is periodic and shares the same period as the oscillator output voltage waveform (see Figure 5.4). In addition, the periodic ISF waveform can be expressed by a Fourier series as follows:

$$
\Gamma\left(\omega_{0} \tau\right)=\frac{c_{0}}{2}+\sum_{n=1}^{\infty} c_{n} \cdot \cos \left(n \cdot \omega_{0} \cdot \tau+\theta_{n}\right)
$$


where " $c_{n}$ " represents the coefficient of the $n^{\text {th }}$ harmonics, " $c_{0}$ " represents the $d c$ component of the Fourier series, and " $\omega_{0}$ " represents the oscillator output frequency. In addition, " $\theta_{n}$ " represents the phase of the $\mathrm{n}^{\text {th }}$ harmonics, but is irrelevant because the noise components (i.e. among all odd and even harmonics) are uncorrelated to each other.

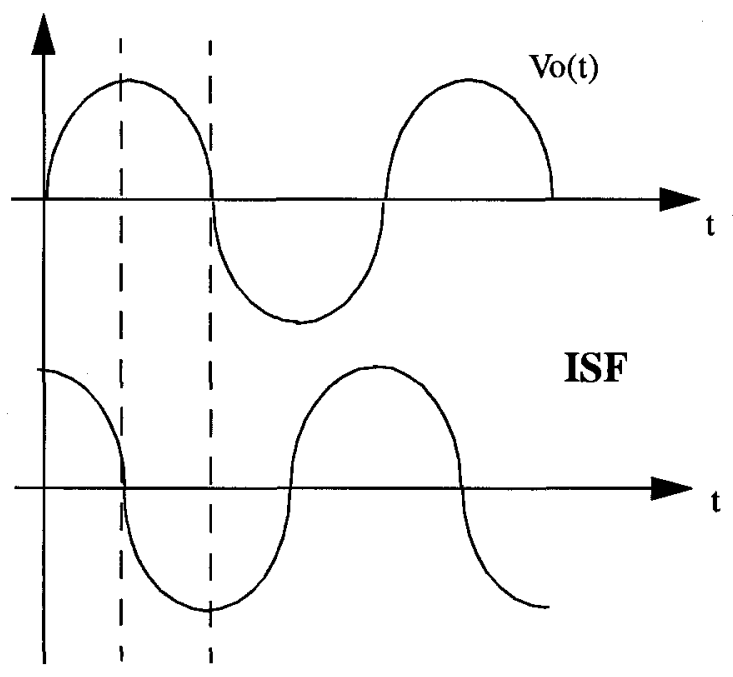

Figure 5.4: Relationship between LC-VCO output voltage and its ISF waveforms

The oscillator output excess phase (i.e. due to a current impulse applied to the circuit) can be calculated as: 


$$
\phi(t)=\frac{1}{q_{\max }} \cdot \int_{-\infty}^{t} \Gamma\left(\omega_{0} \tau\right) \cdot i(\tau) \cdot d \tau=\frac{1}{q_{\max }} \cdot\left[\frac{c_{0}}{2} \cdot \int_{-\infty}^{t} i(\tau) \cdot d \tau+\sum_{n=1}^{\infty} c_{n} \cdot \int_{-\infty}^{t} i(\tau) \cdot \cos \left(n \omega_{0} \tau\right) \cdot d \tau\right]
$$

where " $c_{n}$ " is the coefficient of the nth harmonic inside the Fourier series of the ISF model, and " $\mathrm{q}_{\max }$ " is the total charge induced by the applied current impulse over the $\mathrm{d} \tau$ duration.

If the applied current impulse is located at a low frequency " $\Delta \omega$ " (i.e. as an offset frequency of " $\Delta \omega$ " from $D C)$, then the excess phase shift will be:

$$
\phi(t)=\frac{c_{0}}{2 \cdot q_{\max }} \cdot \int_{-\infty}^{t}\left[I_{0} \cdot \cos (\Delta \omega \tau)\right] d \tau=\frac{I_{0} \cdot c_{0} \cdot \sin (\Delta \omega t)}{2 \cdot q_{\max } \cdot \Delta \omega}
$$

Similar, if the applied current impulse is located at a low frequency offset from the oscillator output frequency, then the excess phase shift will be:

$$
\phi(t)=\frac{c_{1}}{2 \cdot q_{\max }} \cdot \int_{-\infty}^{t}\left\{I_{1} \cdot \cos \left[\left(\omega_{0}+\Delta \omega\right) \tau\right]\right\} d \tau=\frac{I_{1} \cdot c_{1} \cdot \sin (\Delta \omega t)}{2 \cdot q_{\max } \cdot \Delta \omega}
$$


By superposition, the excess phase shift due to all current impulses (i.e. located at a low frequency offset from both odd and even harmonics of the oscillation frequency) can be respresented as:

$$
\phi(t)=\sum_{n=1}^{\infty} \frac{I_{n} \cdot c_{n} \cdot \sin (\Delta \omega t)}{2 \cdot q_{\max } \cdot \Delta \omega}
$$

By substituting Equation 5.29 into Equation 5.23, a pair of sidebands (of same magnitude) appear at an offset of " $\Delta \omega$ " away from the oscillation frequency. The ratio between each of these sidebands and the oscillation output signal can be presented in logscale as:

$$
P_{S B C}(\Delta \omega)=10 \cdot \log \left[\sum_{n=1}^{\infty}\left(\frac{I_{n} \cdot c_{n}}{2 \cdot 2 \cdot q_{\max } \cdot \Delta \omega}\right)\right]^{2}=10 \cdot \log \left[\sum_{n=1}^{\infty}\left(\frac{I_{n} \cdot c_{n}}{4 \cdot q_{\max } \cdot \Delta \omega}\right)\right]^{2}
$$

where the factor " 4 " in the denominator indicates only one of the two sidebands (i.e. mirror images centered around the ideal output signal) is taken into consideration. 
In addition to the current impulse case, Hajimiri further applied his interpretation of this sideband/carrier ratio (or phase noise model) for noise sources with white noise and flicker noise distribution profiles.

For the case of "white" noise current source, the single sideband phase noise can be represented by:

$$
P_{S B C}(\Delta \omega)=10 \cdot \log \left[\sum_{n=1}^{\infty}\left(\frac{I_{n} \cdot c_{n}}{4 \cdot q_{\max } \cdot \Delta \omega}\right)\right]^{2}=10 \cdot \log \left(\frac{\frac{\overline{i_{n}^{2}}}{\Delta f} \cdot \sum_{n=0}^{\infty} c_{n}{ }^{2}}{4 \cdot q_{\max }{ }^{2} \cdot \Delta \omega^{2}}\right)
$$

where the magnitude of the current impulse is now replaced with a constant power spectral density value of " $\mathrm{i}_{\mathrm{n}}{ }^{2} / \Delta \mathrm{f}$ ".

By applying Parseval's relation, the series sum of the ISF Fourier series coefficients can be simplified as:

$$
\sum_{n=0}^{\infty}{c_{n}}^{2}=\frac{1}{\pi} \cdot \int_{0}^{2 \pi}|\Gamma(x)|^{2} d x=2 \cdot \Gamma_{r m s}^{2}
$$


The phase noise model for an oscillator with a white-noise based disturbance can be simplified as:

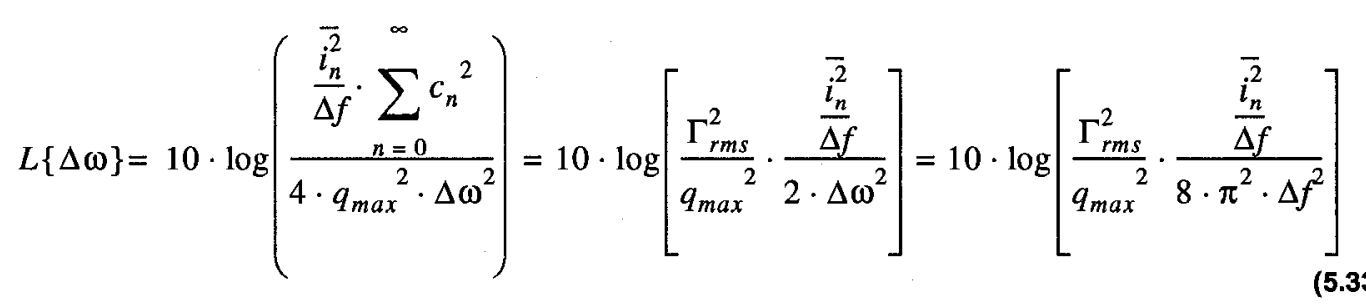

In the above equation, the product of " $\mathrm{i}_{\mathrm{n}}{ }^{2} / \Delta \mathrm{f}$ " and " $\Sigma \mathrm{c}_{\mathrm{n}}{ }^{2}$ " in the nominator refers to the sum of all noise sources close to both odd and even harmonics of the oscillator output

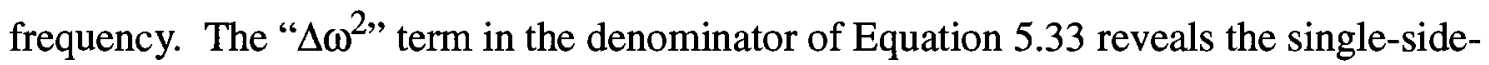
band phase noise varies inversely to the square of the offset frequency; which is similar to the " $1 / \mathrm{f}^{2 "}$ " region of Leeson's phase noise model.

Equations 5.31 and 5.33 reveal the following important properties of Harjimiri's proposed oscillator phase noise model:

- noise near DC gets upconverted by coefficient " $c_{0}$ " onto phase noise near the oscillator circuit output oscillation frequency " $\omega_{0}$ " 
- noise near the oscillator output frequency does not undergo frequency translation, but gets modified by the coefficient " $c_{1}$ "

- noise near both odd and even harmonics of oscillator output oscillation frequency gets down-convertd by coefficients " $c_{n}$ " onto phase noise near the oscillator circuit output oscillation frequency " $\omega_{0}$ "

As for the "flicker" current noise disturbance case, Hajimiri has modelled the current noise source to be:

$$
\frac{\overline{i_{n, 1 / f}^{2}}}{\omega_{1 / f}}=\frac{\overline{i_{n}^{2}}}{\Delta \omega} \rightarrow \overline{i_{n, 1 / f}^{2}}=\overline{i_{n}^{2}} \cdot \frac{\omega_{1 / f}}{\Delta \omega}
$$

Hence, the phase noise model for an oscillator with a flicker-noise based disturbance can be simplified as:

$$
L\{\Delta \omega\}=10 \cdot \log \left(\frac{\overline{i_{n}^{2}} \cdot \frac{\omega_{1 / f}}{\Delta f} \cdot \sum_{n=0}^{\infty} c_{n}^{2}}{4 \cdot q_{\max }^{2} \cdot \Delta \omega^{2}}\right)=10 \cdot \log \left(\frac{\frac{\overline{i_{n}^{2}}}{\Delta f} \cdot \frac{\omega_{1 / f}}{\Delta \omega} \cdot\left(\frac{c_{0}}{2}\right)^{2}}{4 \cdot q_{\max }^{2} \cdot \Delta \omega^{2}}\right)=10 \cdot \log \left(\frac{\left(\frac{c_{0}}{2}\right)^{2}}{q_{\max }^{2}} \cdot \frac{\frac{\overline{i_{n}^{2}}}{\Delta f}}{4 \cdot \Delta \omega^{2}} \cdot \frac{\omega_{1 / f}}{\Delta \omega}\right)
$$


Since flicker noise occurs at very low frequencies, only the " $c_{0} / 2$ " coefficient of the ISF Fourier series (from Equation 5.25) is applicable. In other words, the series sum of " $c_{n}{ }^{2 "}$ coefficients in Equation 5.35 can be replaced with the term " $\left(c_{0} / 2\right)^{2 "}$,

By definition, the $1 / f^{3}$ corner is the location where the $1 / f^{3}$ slope intersects with the $1 / \mathrm{f}^{2}$ slope on an oscillator phase noise plot. Hence the $1 / \mathrm{f}^{3}$ corner can be evaluated by setting Equations 5.33 and 5.35 to an equal value.

$$
\begin{gathered}
L\{\Delta \omega\}=10 \cdot \log \left[\frac{\Gamma_{r m s}^{2}}{q_{\max }^{2}} \cdot \frac{\overline{i_{n}^{2}}}{2 \cdot \Delta \omega^{2}}\right]=10 \cdot \log \left(\frac{\left(\frac{c_{0}}{2}\right)^{2}}{q_{\max }^{2}} \cdot \frac{\overline{i_{n}^{2}}}{4 \cdot \Delta \omega^{2}} \cdot \frac{\omega_{1 / f}}{\Delta \omega}\right) \\
\text { or }\left[\frac{\Gamma_{r m s}^{2}}{q_{\max }^{2}} \cdot \frac{\overline{i_{n}^{2}}}{2 \cdot \Delta \omega^{2}}\right]=\left(\frac{\left(\frac{c_{0}}{2}\right)^{2}}{q_{\max }^{2}} \cdot \frac{\overline{i_{n}^{2}}}{4 \cdot \Delta f} \cdot \frac{\omega_{1 / f}}{\Delta \omega}\right) \rightarrow \Gamma_{r m s}^{2}=\frac{\left(\frac{c_{0}}{2}\right)^{2} \cdot \omega_{1 / f}}{2 \cdot \Delta \omega} \rightarrow \Delta \omega=\frac{\left(\frac{c_{0}}{2}\right)^{2} \cdot \omega_{1 / f}}{2 \cdot \Gamma_{r m s}^{2}}=\left(\frac{\Gamma_{d c}}{\Gamma_{r m s}}\right)^{2} \cdot \frac{\omega_{1 / f}}{2}
\end{gathered}
$$

Hence, the $1 / \mathrm{f}^{3}$ phase noise corner of an oscillator output spectrum is shown not equal to the 1/f device noise corner; but is multiplied by a constant factor. Note that by minimizing the " $\mathrm{c}_{0} / 2$ " coefficient, which is the dc component of the oscillator circuit's 
ISF; one can reduce both the $1 / \mathrm{f}^{3}$ phase noise corner and the oscillator circuit's phase noise (Equations 5.37 and 5.36 respectively).

Hajimiri claimed that one can greatly reduce the $1 / \mathrm{f}^{3}$ phase noise corner by matching the rise and fall times of the oscillator output voltage waveform. The relationship between rise-fall-times-matching and the minimization of the coefficient " $c_{0}$ " is most pronounced in the ring oscillator ISF illustration, which will be covered in the next sub-section.

\subsubsection{Hajimiri's phase noise model (for ring oscillator)}

Hajimiri has further applied his LTV phase noise model to ring type of oscillator circuits [11],[13]. Ring oscillator circuits are revealed to be the most sensitive to a current impulse disturbance during output waveform transitions, which is identical to LC-based oscillator circuits. Similarly, ring oscillators are least sensitive to a current impulse disturbance when their output waveforms are saturated at either the maximum or the minimum voltage levels (see Figure 5.5). 


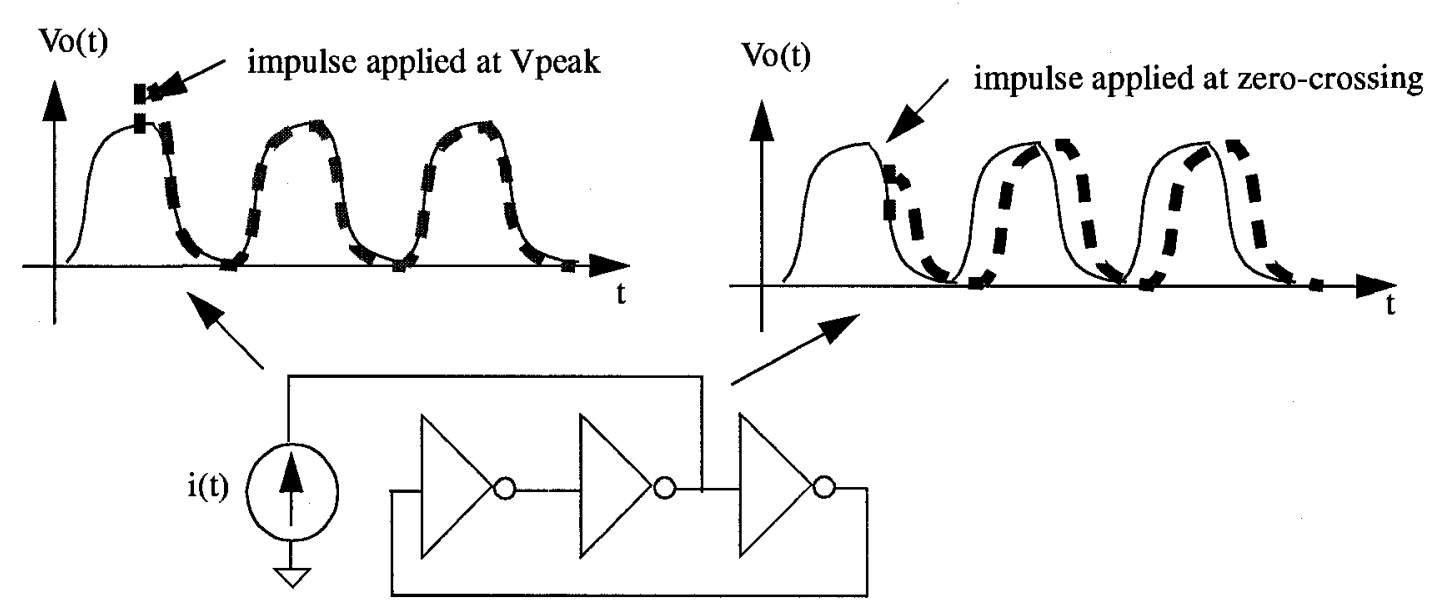

Figure 5.5: Impulse Sensitivity Function of a ring oscillator circuit

Similar to the LC-based oscillators, the ISF of ring oscillator circuits also share the same period with its corresponding ring oscillator output voltage waveform. Since both rise and fall times of a ring oscillator output waveform are shorter than the oscillation period, positive and negative "triangular lobes" on the ring oscillator ISF waveform (Figure 5.6) seem to be spaced out much more than an LC-based oscillator ISF waveform (Figure 5.4).

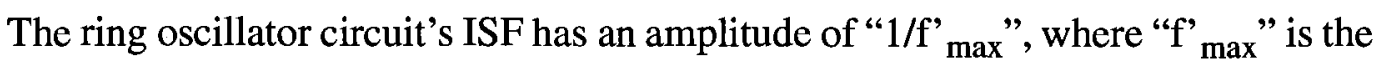
maximum slope of the normalized output voltage waveform (see Figure 5.6). In addition, 
the width of each triangular peak and trough is " $2 / \mathrm{f}$ ' $\max$ ". The rms value of a ring oscillator circuit's ISF can be calculated as the area under a pair of peak and trough (i.e. the time elapsed over one oscillation period):

$$
\Gamma_{r m s}^{2}=\frac{1}{2 \pi} \cdot \int_{0}^{2 \pi} \Gamma^{2}(x) d x=\frac{4}{2 \pi} \cdot \int_{0}^{\frac{1}{f_{\max }}} x^{2} d x=\frac{2}{3 \pi} \cdot\left(\frac{1}{f_{\max }}\right)^{3}
$$

Hence an oscillator circuit's ISF rms value can be greatly reduced if the "f' max" value is maximized, which is the slew rate of the oscillator output voltage waveform. 


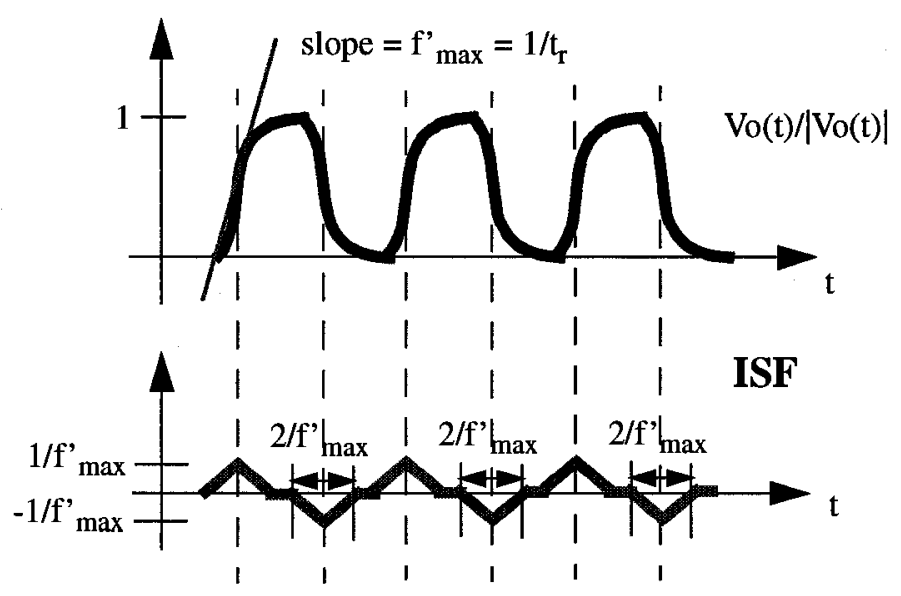

Figure 5.6: Relationship between ring oscillator normalized output voltage and its ISF waveforms

From Figure 5.6, triangular lobes along a ring oscillator circuit's ISF are illustrated to be related to the rise and fall times of the circuit's output voltage waveform. Thus with matching rise and fall times in the oscillator output waveform, areas within all positive and negative triangular lobes cancel each other in the ISF; and the "dc" value of the ISF (or the term " $\mathrm{c}_{0}$ ") will be very close to zero. Otherwise, any mis-matches among the positive and negative lobes in the ISF induce a non-zero "dc" value of the ISF (i.e. through integration of the ISF along the time domain). Although the relationship between risefall-time-matching and the ISF "dc" value is shown with a ring oscillator circuit, this relationship is also applicable to an LC-VCO circuit as well. 
Hajimiri has defined the relationship between the time delay of a single delay stage and the rise time of the output voltage waveform of ring oscillator cirucits to be:

$$
\hat{t}_{D}=\frac{\eta}{f_{\max }}=\eta \cdot t_{r}
$$

where " $n$ " is a proportionality constant with a value close to one. This relationsship is illustrated in Figure 5.7.

Since the output voltage waveform shown in both Figures 5.6 and 5.7 are normalized (i.e. " $\mathrm{V}_{\mathrm{o}}(\mathrm{t}) /\left|\mathrm{V}_{\mathrm{o}}(\mathrm{t})\right|$ ", the slope is approximated to be " $\mathrm{f}$ " ${ }_{\max }$ " or " $1 / \mathrm{t}_{\mathrm{r}}$ " (see Figure 5.7). In other words, the rise time " $t_{r}$ " is speciified as the time for the output waveform to rise from the most negative level to the most positive level of the voltage swing (i.e. $0 \%$ to $100 \%)$ 


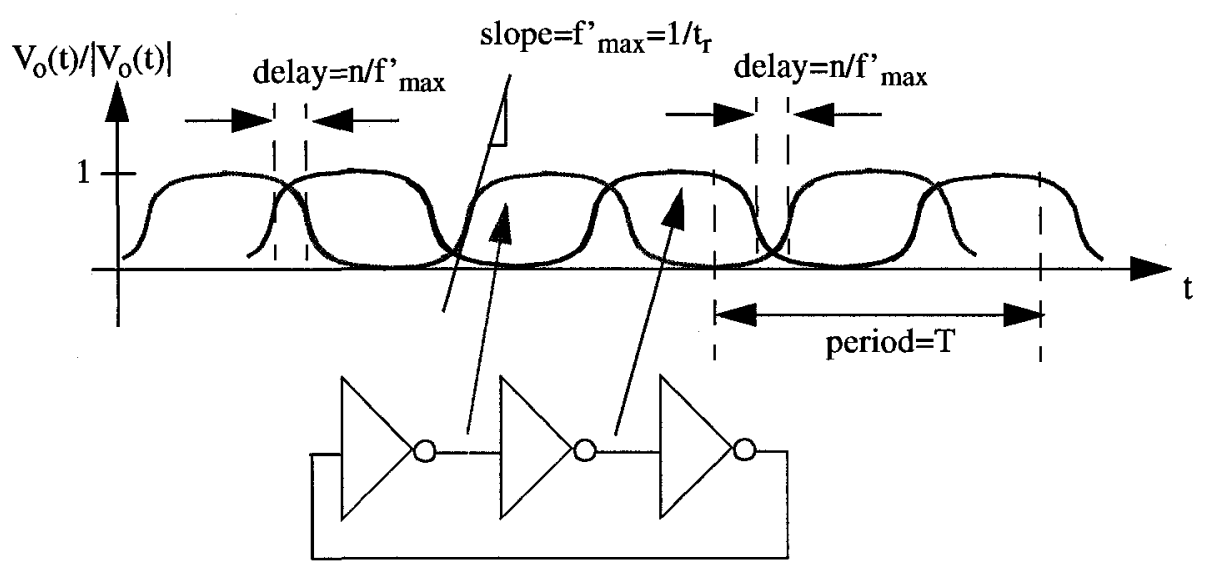

Figure 5.7: Relationship between ring oscillator circuit output signal rise time and delay

Thus for a $\mathrm{N}$ stage ring oscillator circuit, the oscillation signal period (or twice the total propagation delay around the loop) and oscillation frequency will be:

$$
\begin{gathered}
T=2 \pi[\text { radian }]=2 \cdot N \cdot \hat{t}_{D}=\frac{2 \cdot N \cdot \eta}{f_{\max }}=2 \cdot N \cdot \eta \cdot t_{r} \\
f_{o}=\frac{1}{T}=\frac{f_{\max }}{2 \cdot N \cdot \eta}=\frac{1}{2 \cdot N \cdot \eta \cdot t_{r}}
\end{gathered}
$$

For the proposed active-LC-based oscillator circuit, feedback signal paths are differential in nature; and the bias current for each delay stage is defined with a fixed value. Hence the rise time for the oscillation output signal will be: 


$$
t_{r}=\frac{q_{\text {max }}}{I_{\text {tail }}}=\frac{C_{\text {out }} \cdot V_{\text {swing }}}{I_{\text {tail }}}
$$

where " $\mathrm{C}_{\text {out }}$ " is total capacitance at the output node of each oscillator delay stage.

By substituting Equation 5.42 into Equation 5.41, the oscillation frequency of a differential ring oscillator circuit will be:

$$
f_{o}=\frac{1}{T}=\frac{f_{\max }}{2 \cdot N \cdot \eta}=\frac{1}{2 \cdot N \cdot \eta \cdot t_{r}}=\frac{I_{\text {tail }}}{2 \cdot N \cdot \eta \cdot q_{\max }}=\frac{I_{\text {tail }}}{2 \cdot N \cdot \eta \cdot C_{\text {out }} \cdot V_{\text {swing }}}
$$

Hence Equation 5.43 confirms with the operation mechanism of the proposed active LC-VCO circuit, where the choices of " $N$ ", " $\mathrm{C}_{\text {out }}$ ", and " $\mathrm{V}_{\text {swing }}$ " have been finalized upon design completion; and the variation on " $\mathrm{I}_{\text {tail }}$ " directly controls the oscillation frequency.

In Hajimiri's June 1999 journal paper [11], he has specified the drain current noise spectral density of a MOS transistor to be:

$$
\frac{\overline{i_{n}^{2}}}{\Delta f}=4 \cdot k \cdot T \cdot \gamma \cdot \mu \cdot C_{o x} \cdot \frac{W}{L} \cdot\left(V_{G S}-V_{t}\right)=\frac{8 \cdot k \cdot T \cdot \gamma \cdot I_{D S}}{\left(V_{G S}-V_{t}\right)}=\frac{8 \cdot k \cdot T \cdot I_{D S}}{V_{c h a r}}
$$


where

$$
I_{D S}=\frac{1}{2} \cdot \mu \cdot C_{o x} \cdot \frac{W}{L} \cdot\left(V_{G S}-V_{t}\right)^{2}
$$

and

$$
V_{c h a r}=\frac{\left(V_{G S}-V_{t}\right)}{\gamma}
$$

are the drain current formula of a MOS device which operates in the saturation region, and the characteristic voltage of a "long channel" MOS device. The difference between "long" and "short" channel MOS device characteristic voltages is omitted for the phase noise derivation, and will be re-stated afterward.

From Equation 5.44, the total current noise density at an individual oscillator output node will be:

$$
\left(\frac{\overline{i_{n}^{2}}}{\Delta f}\right)_{\text {se }}=\left(\frac{\overline{i_{n}^{2}}}{\Delta f}\right)_{\text {Nswirch }}+\left(\frac{\overline{i_{n}^{2}}}{\Delta f}\right)_{\text {Nlatch }}+\left(\frac{\overline{i_{n}^{2}}}{\Delta f}\right)_{\text {Pload }}=8 \cdot k \cdot T \cdot\left(\frac{I_{N s w i t c h}}{V_{\text {charN }}}+\frac{I_{\text {tail }}-I_{\text {Nswitch }}}{V_{\text {charN }}}+\frac{I_{\text {tail }}}{V_{\text {charP }}}\right)
$$

or 


$$
\left(\frac{\overline{i_{n}^{2}}}{\Delta f}\right)_{s e}=\left(\frac{\overline{i_{n}^{2}}}{\Delta f}\right)_{\text {Nswitch }}+\left(\frac{\overline{i_{n}^{2}}}{\Delta f}\right)_{\text {Nlatch }}+\left(\frac{\overline{i_{n}^{2}}}{\Delta f}\right)_{\text {Pload }}=8 \cdot k \cdot T \cdot I_{\text {tail }} \cdot\left(\frac{1}{V_{\text {charN }}}+\frac{1}{V_{\text {charP }}}\right)
$$

Equation 5.47 is valid because the total bias current is split between the NMOS switching-pair and NMOS cross-coupled-latch devices.

Thus the total current noise density for the entire $\mathrm{N}$-stage differential ring oscillator will be:

$$
\frac{\overline{i_{n}^{2}}}{\Delta f}=2 \cdot N \cdot\left(\frac{\bar{i}_{n}^{2}}{\Delta f}\right)_{s e}=16 \cdot N \cdot k \cdot T \cdot I_{\text {tail }} \cdot\left(\frac{1}{V_{\text {char } N}}+\frac{1}{V_{\text {charP }}}\right)
$$

By substituting Equations 5.49 and 5.56 into Equation 5.33, the phase noise of a $\mathrm{N}$-stage differential ring oscillator circuit can be calculated as:

$$
L\{\Delta \omega\}=10 \cdot \log \left[\frac{\Gamma_{r m s}^{2}}{q_{\max }^{2}} \cdot \frac{\overline{i_{n}^{2}}}{8 f \cdot \pi^{2} \cdot \Delta f^{2}}\right]=10 \cdot \log \left[\frac{\left(\frac{2 \cdot \pi^{2}}{3 \cdot \eta^{3} \cdot N^{3}}\right)}{\left(\frac{I_{\text {tail }}}{4 \cdot \eta^{2} \cdot N^{2} \cdot f_{0}^{2}}\right)} \cdot \frac{\left(16 \cdot N k T \cdot I_{\text {tail }}\right) \cdot\left(\frac{1}{V_{\text {char } N}}+\frac{1}{V_{\text {charP }}}\right)}{8 \cdot \pi^{2} \cdot \Delta f^{2}}\right]
$$


or

$$
L\{\Delta \omega\}=10 \cdot \log \left[\frac{16}{3 \cdot \eta} \cdot \frac{N \cdot k \cdot T}{P} \cdot\left(\frac{V_{D D}}{V_{\text {char } N}}+\frac{V_{D D}}{V_{c h a r P}}\right) \cdot\left(\frac{f_{0}}{\Delta f}\right)^{2}\right]
$$

where

$$
P=N \cdot I_{\text {tail }} \cdot V_{D D}
$$

and

$$
V_{c h a r}=\frac{E_{c} \cdot L}{\gamma}
$$

are the total power dissipation of the $\mathrm{N}$-stage differential ring oscillator circuit, and the characteristic voltage of the "short channel" MOS device respectively.

Equation 5.53 is applicable to devices classified in the "short channel" regime, as minimum gate lengths of 0.18 um were chosen for NMOS devices (and 0.2 um were chosen for PMOS devices) in the proposed differential ring oscillator circuits. In addition, " $E_{\mathrm{c}}$ " is the critical electric field, and is defined as the value of electrical field that halves the velocity of carriers inside the NMOS device; and " $L$ " is the gate length of the NMOS device. Since the characteristic voltage of the MOS device varies directly with the corresponding device gate length, shorter gate length devices lead to smaller $\mathrm{V}_{\text {char }}$ values, 
which increases the oscillator cirucit's phase noise given by Equation 5.51. Hence there is a tradeoff between speed and phase noise performance of a differential ring oscillator circuit.

From Equation 5.51, the phase noise of a differential ring oscillator circuit is revealed to be dependent on the number of delay stages " $N$ "; which agrees with Hajimiri's comments in his June 1999 journal [11].

\subsection{Performance of Active LC-VCO}

The 2-stage active-LC-voltage-controlled-oscillator circuit has been simulated with parasitic capacitance extracted from their corresponding layout. Since the frequency tuning circuit is implemented with differential input controls, the voltage levels assigned to the two input pins, namely "Vupb" and "Vdnb", vary inversely (see Figure 5.8). 

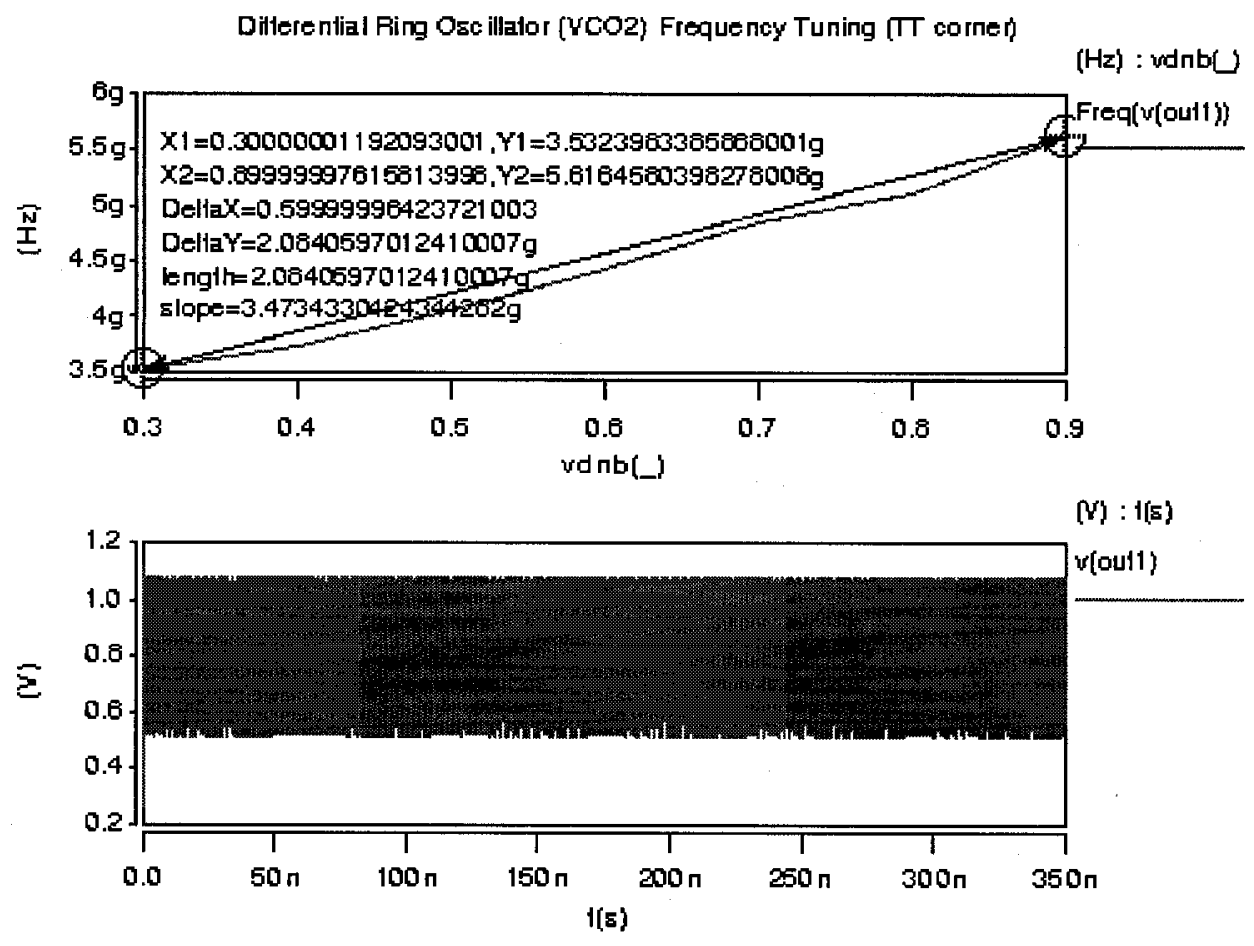

Figure 5.8: Simulated results of active LC-VCO frequency tuning

Both simulation results and measurements are taken from one of the two output nodes of the 3-stage differential buffer circuits, and summarized in the following table: 
TABLE 5.1. Post layout simulation results and lab measurements of active LC-VCO circuit

\begin{tabular}{|c|c|c|c|c|c|c|c|}
\hline Vupb & Vdnb & $\begin{array}{l}\text { fvco } \\
\text { (sim) }\end{array}$ & $\begin{array}{l}\text { fvco } \\
\text { (meas) }\end{array}$ & $\begin{array}{l}\text { Phase Noise } \\
\text { (sim at } \\
\text { fvco+100kHz } \\
\text { ) }\end{array}$ & $\begin{array}{l}\text { Phase Noise } \\
\text { (sim at } \\
\text { fveo+1MHz) }\end{array}$ & $\begin{array}{l}\text { Phase Noise } \\
\text { (meas at } \\
\text { fvco+100kHz } \\
\text { ) }\end{array}$ & $\begin{array}{l}\text { Phase Noise } \\
\text { (meas at } \\
\text { fveo+1MHz) }\end{array}$ \\
\hline $0.9 \mathrm{~V}$ & $0.3 \mathrm{~V}$ & $3.532 \mathrm{GHz}$ & $3.292 \mathrm{GHz}$ & $-61.21 \mathrm{dBc} / \mathrm{Hz}$ & $-81.04 \mathrm{dBc} / \mathrm{Hz}$ & $-62.64 \mathrm{dBc} / \mathrm{Hz}$ & $-64.34 \mathrm{dBc} / \mathrm{Hz}$ \\
\hline $0.8 \mathrm{~V}$ & $0.4 \mathrm{~V}$ & $3.735 \mathrm{GHz}$ & $3.577 \mathrm{GHz}$ & $-61.10 \mathrm{dBc} / \mathrm{Hz}$ & $-80.83 \mathrm{dBc} / \mathrm{Hz}$ & $-58.52 \mathrm{dBc} / \mathrm{Hz}$ & $-59.08 \mathrm{dBc} / \mathrm{Hz}$ \\
\hline $0.7 \mathrm{~V}$ & $0.5 \mathrm{~V}$ & $4.034 \mathrm{GHz}$ & $3.995 \mathrm{GHz}$ & $-60.94 \mathrm{dBc} / \mathrm{Hz}$ & $-80.40 \mathrm{dBc} / \mathrm{Hz}$ & $-59.83 \mathrm{dBc} / \mathrm{Hz}$ & $-61.52 \mathrm{dBc} / \mathrm{Hz}$ \\
\hline $0.6 \mathrm{~V}$ & $0.6 \mathrm{~V}$ & $4.433 \mathrm{GHz}$ & $4.515 \mathrm{GHz}$ & $-60.77 \mathrm{dBc} / \mathrm{Hz}$ & $-79.49 \mathrm{dBc} / \mathrm{Hz}$ & $-63.62 \mathrm{dBc} / \mathrm{Hz}$ & $-66.80 \mathrm{dBc} / \mathrm{Hz}$ \\
\hline $0.5 \mathrm{~V}$ & $0.7 \mathrm{~V}$ & $4.849 \mathrm{GHz}$ & $5.167 \mathrm{GHz}$ & $-60.57 \mathrm{dBc} / \mathrm{Hz}$ & $-77.14 \mathrm{dBc} / \mathrm{Hz}$ & $-62.24 \mathrm{dBc} / \mathrm{Hz}$ & $-67.23 \mathrm{dBc} / \mathrm{Hz}$ \\
\hline $0.4 \mathrm{~V}$ & $0.8 \mathrm{~V}$ & $5.193 \mathrm{GHz}$ & & $-60.17 \mathrm{dBc} / \mathrm{Hz}$ & $-72.35 \mathrm{dBc} / \mathrm{Hz}$ & & \\
\hline $0.3 \mathrm{~V}$ & $0.9 \mathrm{~V}$ & $5.607 \mathrm{GHz}$ & & $-60.23 \mathrm{dBc} / \mathrm{Hz}$ & $-72.37 \mathrm{dBc} / \mathrm{Hz}$ & & \\
\hline
\end{tabular}

From the above table, three major differences between simulation and measured results are the minimum/centre/maximum frequencies, the $\mathrm{VCO}$ gain (or $\mathrm{K}_{\mathrm{VCO}}$ ), and the phase noise of the oscillator circuit.

From simulations, the oscillator output frequency has a minimum, a centre, and a maximum value of $3.532 \mathrm{GHz}, 4.5695 \mathrm{GHz}$, and $5.607 \mathrm{GHz}$; which corresponds to "Vdnb" control voltage values of $0.3 \mathrm{~V}, 0.6 \mathrm{~V}$, and $0.9 \mathrm{~V}$ respectively. From lab measurements, the oscillator output frequency has a minimum, a centre, and a maximum value of $3.292 \mathrm{GHz}$, 4.2295GHz, and 5.167GHz; which corresponds to "Vdnb" control voltage values of $0.3 \mathrm{~V}$, $0.5 \mathrm{~V}$, and $0.7 \mathrm{~V}$ respectively. The difference between simulation and measured results 
may be caused by two factors: incomplete post-layout netlist extraction and inaccurate voltage-to-current converter modeling.

The simulated post-layout netlists are extracted from both oscillator and buffer circuits, but the parasitics from inter-connections between the oscillator and buffer circuits is missing; which contributes to the difference in both minimum and maximum oscillation frequencies between simulations and measurements.

At the "Vdnb $=0.5 \mathrm{~V}, \mathrm{Vupb}=0.7 \mathrm{~V}$ " setting, the measured oscillation frequency is closest to the corresponding simulated result. But when "Vdnb" is set to lower voltage levels, the measured oscillation frequencies are lower than the simulated results. Similarly, when "Vdnb" is set to voltage levels higher than $0.5 \mathrm{~V}$, the measured oscillation frequencies are higher than the simulated results. Based on the measured results, the voltageto-current converter circuit steers more current to the ring oscillator circuit under each $100 \mathrm{mV}$ change in the control voltages. In other words, the implemented voltage-to-current converter circuit has higher conversion gain than the simulated version.

Furthermore, this characteristics is also reflected in the VCO gain values (or $\mathrm{KVCO}$ ) between simulated and measured results. The simulated VCO gain transfer func- 
tion (or $\mathrm{K}_{\mathrm{VCO}}$ ) value is about $3.4734 \mathrm{GHz} / \mathrm{V}$. The measured maximum and minimum oscillation frequencies are $5.167 \mathrm{GHz}$ and $3.292 \mathrm{GHz}$, which corresponds to a $400 \mathrm{mV}$ change in the control voltage. Thus the measured KVCO value can be approximated as $(5.167 \mathrm{GHz}-3.292 \mathrm{GHz}) / 0.4 \mathrm{~V}$ or $4.6875 \mathrm{GHz} / \mathrm{V}$.

The phase noise results at $100 \mathrm{kHz}$ away from each oscillation frequency setting are close between simulations and measurements. On the other hand, phase noise results at $1 \mathrm{MHz}$ away from each oscillation frequency setting are very different between simulations and measuremnets. From simulations, the phase noise difference between $100 \mathrm{kHz}$ and $1 \mathrm{MHz}$ offset frequencies are about $20 \mathrm{~dB}$ at each oscillation setting. This follows region 8 of Leeson's phase noise model; which has a slope of $-20 \mathrm{~dB} / \mathrm{decade}$. From measurements, the phase noise at $1 \mathrm{MHz}$ offset frequency is almost identical to the phase noise at $100 \mathrm{kHz}$ offset frequency. This may due to noise and/or common mode interference coming from the power supply. Due to the pad-limited layout approach, only one of the differential buffer output is wirebonded to the off-chip environment. Hence the oscillator's phase noise performance at the off-chip load cannot benefit from the common mode rejection property of the differential circuit topology. In addition, on-chip decoupling capacitors are missing between power and ground buses; hence any noise on the on-chip 
power bus cannot be filtered out properly. Unfortunately, we cannot verify these claims because phase noise measurements can only be taken at one of the differential buffer output nodes; and the effect from the buffer circuit cannot be de-embedded.

\subsubsection{Impact on phase noise: choice of load}

The simulated output voltage waveforms are revealed to have asymmetrical rise and fall times. This mismatch leads to a widened $1 / \mathrm{f}^{3}$ corner frequency in the oscillator output spectrum, and consequently a relatively high phase noise profile (i.e. phase noise at $100 \mathrm{kHz}$ away from both simulated and measured oscillation frequency). Hajimiri has suggested the use of more linear loads to resolve this issue.

If poly-resistors or triode-mode PMOS devices are used as loads, then variations across process, voltage, and temperature (or simply PVT) only leads to a variation of the "resistance" value of the linear loads; which is much smaller than the resistivity difference between a triode-mode and a saturation-mode PMOS transistor. Although simulations on the existing PMOS active loads (i.e. operating in saturation mode) have been launched for "typical" and "slow" PVT simulation setups, there is a risk of changing PMOS operating 
regions for "skew" process corner (i.e. "fast-P/slow-N"). Sometimes, skew corner process data may not be accessible from the chosen implementation process; and this type of potential risk may not be revealed through simulations.

In addition to the elimination of PMOS active load operation mode variations, the selection of linear loads (i.e. poly-resistor or triode-mode PMOS device) also eliminates the need for the PMOS current mirror circuit. Hence less current density noise sources exist in the differential ring oscillator circuit, and the phase noise performance should be improved as well. If consistent output voltage magnitude is needed across PVT variations, a common-mode feed-back loop (or CMFB) circuit can be utilized to meet this requirement. CMFB circuit is a negative feed-back loop that involves with an operational amplifier, a voltage reference branch, and a sense circuit embedded in the differential ring oscillator which provides the needed common-mode voltage level information.

Finally, the number of delay stages inside a differential ring oscillator circuit affects both $1 / \mathrm{f}^{3}$ corner frequency and the phase noise performance of the oscillator circuit. Derivation of this relationship is reviewed in the next sub-section. 


\subsubsection{Impact on phase noise: number of delay stages and flicker noise up-conversion}

In addition to the down-conversion of noise close to harmonics of the oscillator output frequency, the number of delay stages in a ring oscillator circuit is revealed to be related to an oscillator's ISF rms value. From Figure 5.6, the sum of a pair of ISF positive and negative triangular lobes is:

$$
\Gamma_{r m s}^{2}=\frac{1}{2 \pi} \cdot \int_{0}^{2 \pi} \Gamma^{2}(t) d t=\frac{4}{2 \pi} \cdot \int_{0}^{\frac{1}{\rho_{\max }}} t^{2} d t=\frac{2}{3 \pi} \cdot\left(\frac{1}{f_{\max }}\right)^{3}
$$

From Equation 5.40:

$$
T=2 \pi[\text { radian }]=2 \cdot N \cdot \hat{t}_{D}=\frac{2 \cdot N \cdot \eta}{f_{\max }} \rightarrow \frac{1}{f_{\max }}=\frac{\pi}{N \cdot \eta}
$$

By substituting Equation 5.55 back into Equation 5.54:

$$
\Gamma_{r m s}^{2}=\frac{2}{3 \pi} \cdot\left(\frac{1}{f_{m a x}}\right)^{3}=\frac{2}{3 \pi} \cdot\left(\frac{\pi}{N \cdot \eta}\right)^{3}=\frac{2 \pi^{2}}{3 \cdot N^{3} \cdot \eta^{3}} \rightarrow \Gamma_{r m s}=\sqrt{\frac{2 \pi^{2}}{3 \cdot \eta^{3}}} \cdot\left(\frac{1}{N}\right)^{\frac{3}{2}}
$$

Since the relationship of the $1 / \mathrm{f}^{3}$ corner on the oscillator output phase noise profile is related to both dc and rms values of the ISF: 


$$
f_{1 / f^{3}}=f_{1 / f} \cdot \frac{\Gamma_{d c}^{2}}{\Gamma_{r m s}^{2}}=f_{1 / f} \cdot \Gamma_{d c}^{2} \cdot \frac{3 \cdot N^{3} \cdot \eta^{3}}{2 \pi^{2}}
$$

the $1 / \mathrm{f}^{3}$ corner of a differential ring oscillator increases nonlinearly with the number of delay stages. Due to the squared "oscillator's ISF dc value" term; the $1 / \mathrm{f}^{3}$ corner can be reduced by matching both rise and fall times of the oscillator output voltage waveform.

This relationship reveals that differential ring oscillator circuits are more susceptable to the " $1 / \mathrm{f}$ noise upconversion to phase noise" mechanism as the number of delay stages increases. Furthermore, with the widened $1 / \mathrm{f}^{3}$ corner, phase noise performance of the proposed 2-stage differential ring oscillator circuit is expected to be worse than the proposed single-stage passive LC-VCO circuit; which agrees with both post-layout simulation and measured results.

\subsection{Performance of the Passive LC-VCO}

The passive LC-VCO circuit is simulated with parasitics extracted from the circuit layout. As discussed in Chapter 2, eight shunt-connected varactors are implemented as 
the frequency tuning element for this oscillator circuit; and the voltage applied to the input control pin, "Vtune", is varied from $0.2 \mathrm{~V}$ to $1.8 \mathrm{~V}$ (see Figure 5.9).
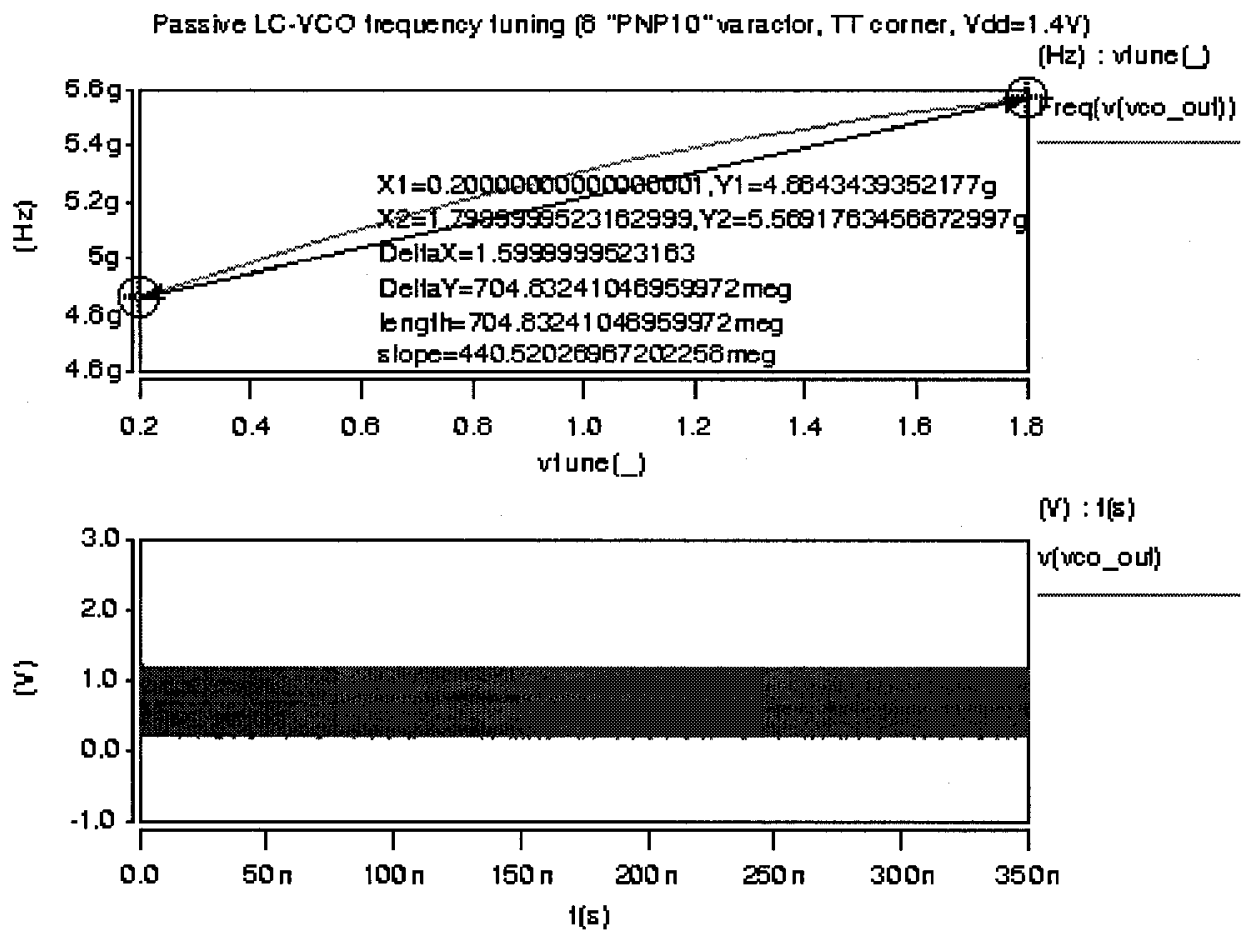

Figure 5.9: Simulated results of passive LC-VCO frequency tuning

Both simulation results and measurements are taken from one of the two output nodes of the 3-stage differential buffer circuits, and summarized in the following table: 
TABLE 5.2. Post layout simulation results and lab measurements of passive LC-VCO circuit

\begin{tabular}{|c|c|c|c|c|c|c|}
\hline dnb & fvco (sim) & $\begin{array}{l}\text { fvco } \\
\text { (meas) }\end{array}$ & $\begin{array}{l}\text { Phase Noise } \\
\text { (sim at } \\
\text { fvco+100kHz) }\end{array}$ & $\begin{array}{l}\text { Phase Noise } \\
\text { (sim at } \\
\text { fvco+1MHz) }\end{array}$ & $\begin{array}{l}\text { Phase Noise } \\
\text { (meas at } \\
\text { fvco+100kHz) }\end{array}$ & $\begin{array}{l}\text { Phase Noise } \\
\text { (meas at } \\
\text { fvco+1MHz) }\end{array}$ \\
\hline $0.2 \mathrm{~V}$ & $4.864 \mathrm{GHz}$ & $4.575 \mathrm{GHz}$ & $-78.927 \mathrm{dBc} / \mathrm{Hz}$ & $-105.58 \mathrm{dBc} / \mathrm{Hz}$ & $-62.49 \mathrm{dBc} / \mathrm{Hz}$ & $-65.11 \mathrm{dBc} / \mathrm{Hz}$ \\
\hline $0.3 \mathrm{~V}$ & $4.923 \mathrm{GHz}$ & $4.710 \mathrm{GHz}$ & $-81.277 \mathrm{dBc} / \mathrm{Hz}$ & $-107.74 \mathrm{dBc} / \mathrm{Hz}$ & $-61.99 \mathrm{dBc} / \mathrm{Hz}$ & $-69.36 \mathrm{dBc} / \mathrm{Hz}$ \\
\hline $0.4 \mathrm{~V}$ & $4.978 \mathrm{GHz}$ & $4.815 \mathrm{GHz}$ & $-83.425 \mathrm{dBc} / \mathrm{Hz}$ & $-109.19 \mathrm{dBc} / \mathrm{Hz}$ & $-66.54 \mathrm{dBc} / \mathrm{Hz}$ & $-70.68 \mathrm{dBc} / \mathrm{Hz}$ \\
\hline $0.5 \mathrm{~V}$ & $5.040 \mathrm{GHz}$ & $4.905 \mathrm{GHz}$ & $-82.286 \mathrm{dBc} / \mathrm{Hz}$ & $-108.66 \mathrm{dBc} / \mathrm{Hz}$ & $-66.42 \mathrm{dBc} / \mathrm{Hz}$ & $-70.68 \mathrm{dBc} / \mathrm{Hz}$ \\
\hline $0.6 \mathrm{~V}$ & $5.098 \mathrm{GHz}$ & $4.965 \mathrm{GHz}$ & $-81.226 \mathrm{dBc} / \mathrm{Hz}$ & $-106.05 \mathrm{dBc} / \mathrm{Hz}$ & $-66.36 \mathrm{dBc} / \mathrm{Hz}$ & $-70.82 \mathrm{dBc} / \mathrm{Hz}$ \\
\hline $0.7 \mathrm{~V}$ & $5.156 \mathrm{GHz}$ & $5.020 \mathrm{GHz}$ & $-77.673 \mathrm{dBc} / \mathrm{Hz}$ & $-105.47 \mathrm{dBc} / \mathrm{Hz}$ & $-65.69 \mathrm{dBc} / \mathrm{Hz}$ & $-73.89 \mathrm{dBc} / \mathrm{Hz}$ \\
\hline $0.8 \mathrm{~V}$ & $5.209 \mathrm{GHz}$ & $5.070 \mathrm{GHz}$ & $-76.515 \mathrm{dBc} / \mathrm{Hz}$ & $-104.52 \mathrm{dBc} / \mathrm{Hz}$ & $-66.50 \mathrm{dBc} / \mathrm{Hz}$ & $-74.83 \mathrm{dBc} / \mathrm{Hz}$ \\
\hline $0.9 \mathrm{~V}$ & $5.260 \mathrm{GHz}$ & $5.110 \mathrm{GHz}$ & $-75.812 \mathrm{dBc} / \mathrm{Hz}$ & $-103.92 \mathrm{dBc} / \mathrm{Hz}$ & $-63.67 \mathrm{dBc} / \mathrm{Hz}$ & $-65.53 \mathrm{dBc} / \mathrm{Hz}$ \\
\hline $1.0 \mathrm{~V}$ & $5.308 \mathrm{GHz}$ & $5.150 \mathrm{GHz}$ & $-75.292 \mathrm{dBc} / \mathrm{Hz}$ & $-103.47 \mathrm{dBc} / \mathrm{Hz}$ & $-63.88 \mathrm{dBc} / \mathrm{Hz}$ & $-71.25 \mathrm{dBc} / \mathrm{Hz}$ \\
\hline $1.1 \mathrm{~V}$ & $5.351 \mathrm{GHz}$ & $5.180 \mathrm{GHz}$ & $-74.906 \mathrm{dBc} / \mathrm{Hz}$ & $-103.13 \mathrm{dBc} / \mathrm{Hz}$ & $-62.74 \mathrm{dBc} / \mathrm{Hz}$ & $-68.97 \mathrm{dBc} / \mathrm{Hz}$ \\
\hline $1.2 \mathrm{~V}$ & $5.391 \mathrm{GHz}$ & $5.215 \mathrm{GHz}$ & $-74.607 \mathrm{dBc} / \mathrm{Hz}$ & $-102.86 \mathrm{dBc} / \mathrm{Hz}$ & $-63.00 \mathrm{dBc} / \mathrm{Hz}$ & $-70.39 \mathrm{dBc} / \mathrm{Hz}$ \\
\hline $1.3 \mathrm{~V}$ & $5.427 \mathrm{GHz}$ & $5.240 \mathrm{GHz}$ & $-74.364 \mathrm{dBc} / \mathrm{Hz}$ & $-102.65 \mathrm{dBc} / \mathrm{Hz}$ & $-66.72 \mathrm{dBc} / \mathrm{Hz}$ & $-74.37 \mathrm{dBc} / \mathrm{Hz}$ \\
\hline $1.4 \mathrm{~V}$ & $5.461 \mathrm{GHz}$ & $5.270 \mathrm{GHz}$ & $-74.162 \mathrm{dBc} / \mathrm{Hz}$ & $-102.47 \mathrm{dBc} / \mathrm{Hz}$ & $-66.57 \mathrm{dBc} / \mathrm{Hz}$ & $-74.15 \mathrm{dBc} / \mathrm{Hz}$ \\
\hline $1.5 \mathrm{~V}$ & $5.491 \mathrm{GHz}$ & $5.290 \mathrm{GHz}$ & $-73.990 \mathrm{dBc} / \mathrm{Hz}$ & $-102.31 \mathrm{dBc} / \mathrm{Hz}$ & $-61.26 \mathrm{dBc} / \mathrm{Hz}$ & $-70.86 \mathrm{dBc} / \mathrm{Hz}$ \\
\hline $1.6 \mathrm{~V}$ & $5.517 \mathrm{GHz}$ & $5.315 \mathrm{GHz}$ & $-73.842 \mathrm{dBc} / \mathrm{Hz}$ & $-102.18 \mathrm{dBc} / \mathrm{Hz}$ & $-57.47 \mathrm{dBc} / \mathrm{Hz}$ & $-65.77 \mathrm{dBc} / \mathrm{Hz}$ \\
\hline $1.7 \mathrm{~V}$ & $5.544 \mathrm{GHz}$ & $5.335 \mathrm{GHz}$ & $-73.712 \mathrm{dBc} / \mathrm{Hz}$ & $-102.06 \mathrm{dBc} / \mathrm{Hz}$ & $-59.40 \mathrm{dBc} / \mathrm{Hz}$ & $-68.07 \mathrm{dBc} / \mathrm{Hz}$ \\
\hline $1.8 \mathrm{~V}$ & $5.569 \mathrm{GHz}$ & $5.360 \mathrm{GHz}$ & $-73.596 \mathrm{dBc} / \mathrm{Hz}$ & $-101.95 \mathrm{dBc} / \mathrm{Hz}$ & $-63.37 \mathrm{dBc} / \mathrm{Hz}$ & $-72.38 \mathrm{dBc} / \mathrm{Hz}$ \\
\hline
\end{tabular}

From the above table, three major differences between simulation and measured results are the minimum/centre/maximum frequencies, the $\mathrm{VCO}$ gain (or $\mathrm{K}_{\mathrm{VCO}}$ ), and the phase noise of the oscillator circuit.

From simulations, the oscillator output frequency has a minimum, a centre, and a maximum value of $4.864 \mathrm{GHz}, 5.2165 \mathrm{GHz}$, and $5.569 \mathrm{GHz}$; which corresponds to "Vtune" 
control voltage values of $0.2 \mathrm{~V}, 1.0 \mathrm{~V}$, and $1.8 \mathrm{~V}$ respectively. From lab measurements, the oscillator output frequency has a minimum, a centre, and a maximum value of $4.575 \mathrm{GHz}$, 4.9675GHz, and 5.36GHz; which corresponds to the same "Vtune" settings. Similar to the active LC-VCO simulations, the difference between simulation and measured results of the passive LC-VCO circuit is due to the missing parasitics from inter-connections between the oscillator and buffer circuits; which contributes to the difference in the minimum, the centre, and the maximum oscillation frequencies between simulations and measurements.

When the control voltage "Vtune" is set to a voltage level of $0.5 \mathrm{~V}$ and higher, the incremental output oscillation frequency is very close between simulation and measured results; and the PNP varactors seem to behave closely to the simulated device model. But for "Vtune" with settings between $0.2 \mathrm{~V}$ and $0.4 \mathrm{~V}$, measured incremental output oscillation frequencies are higher than the corresponding simulated values. This may be caused by some inaccuracy of the varactor device model. Hence the overall LC-VCO circuit's output frequency variation is larger than the simulated version, which leads to a slightly higher $\mathrm{K}_{\mathrm{VCO}}$ value. The simulated $\mathrm{K}_{\mathrm{VCO}}$ value is about $440.62 \mathrm{MHz} / \mathrm{V}$. The measured maximum and minimum oscillation frequencies are $5.36 \mathrm{GHz}$ and $4.575 \mathrm{GHz}$, which corre- 
sponds to a $1.6 \mathrm{~V}$ change in the control voltage. Thus the measured KVCO value can be approximated as $(5.36 \mathrm{GHz}-4.575 \mathrm{GHz}) / 1.6 \mathrm{~V}$ or $490.525 \mathrm{MHz} / \mathrm{V}$. In other words, the implemented passive LC-VCO circuit has an output frequency tuning range of $815 \mathrm{MHz}$.

From simulations, the phase noise difference between $100 \mathrm{kHz}$ and $1 \mathrm{MHz}$ offset frequencies are about $26 \mathrm{~dB}$ at each oscillation setting. This falls into regions 7 and 8 of Leeson's phase noise model; which has a slope of $-30 \mathrm{~dB} / \mathrm{decade}$ and $-20 \mathrm{~dB} / \mathrm{decade}$ respectively. From measurements, the phase noise at $1 \mathrm{MHz}$ offset frequency is almost identical to the phase noise at $100 \mathrm{kHz}$ offset frequency. Similar to the active LC-VCO circuit setup, the poor phase noise performance may be caused by both single-ended output signal measurement and the lack of on-chip decoupling capacitors between power and ground buses. Similar to the active LC-VCO situation, we cannot test both oscillator and buffer circuits separately to verify these claims.

\subsubsection{Impact on phase noise: asymmetrical rise and fall times}

Similar to the active LC-VCO cirucits, the oscillator outupt voltage waveform has slight asymmetry between rise and fall times; hence there exists a small non-zero value for 
the oscillator's ISF dc value. This mismatch in waveform rise and fall times leads to a relatively high $1 / \mathrm{f}^{3}$ corner frequency in the oscillator's output spectrum, and a higher phase noise profile. The solution to this issue is to re-balance the drive strength of both PMOS and NMOS switching devices (i.e. the two cross-coupled current starved CMOS inverter) inside the -gm gain stage of the oscillator circuit:

$$
g_{m n}=\sqrt{2 \cdot \mu_{n} \cdot C_{o x} \cdot \frac{W_{n}}{L_{n}} \cdot I_{D S}}=g_{m p}=\sqrt{2 \cdot \mu_{p} \cdot C_{o x} \cdot \frac{W_{p}}{L_{p}} \cdot I_{D S}} \rightarrow \mu_{n} \cdot \frac{W_{n}}{L_{n}}=\mu_{p} \cdot \frac{W_{p}}{L_{p}}
$$

In addition to the " $\mathrm{g}_{\mathrm{m}}$ " matching among the PMOS and NMOS devices, Dr. Calvin Plett and Dr. John Rogers have suggested the idea of "impedance" matching for further improvement on the oscillator circuit's phase noise performance [29]. Hence the total gate area of both PMOS and NMOS devices can be matched as follows:

$$
C_{g n}=\gamma \cdot W_{n} \cdot L_{n} \cdot C_{o x}=C_{g p}=\gamma \cdot W_{p} \cdot L_{p} \cdot C_{o x} \rightarrow W_{n} \cdot L_{n}=W_{p} \cdot L_{p}
$$

where " $\gamma$ " represents the fraction of the MOS device gate that is not in the "pinch-off" region. Hence " $\gamma$ " has a value of $2 / 3$ and 1 when the "long-channel" MOS device is oper- 
ating in "saturation" and "triode" modes respectively. In this case, $\mathrm{V}_{\mathrm{DS}}$ must be $0 \mathrm{~V}$ when the NMOS device is operating in "triode" mode. For "short-channel" MOS devices, " $"$ " has a value close to 1 when the transistor is operating in the "saturation" mode.

By subsituting Equation 5.59 into Equation 5.58:

$$
\mu_{n} \cdot \frac{W_{n}}{L_{n}}=\mu_{p} \cdot \frac{W_{p}}{L_{p}} \rightarrow L_{n}=L_{p} \cdot \frac{\mu_{n} \cdot W_{n}}{\mu_{p} \cdot W_{p}}=L_{p} \cdot \frac{\mu_{n}}{\mu_{p}} \cdot \frac{L_{p}}{L_{n}} \rightarrow L_{n}^{2}=L_{p}^{2} \cdot \frac{\mu_{n}}{\mu_{p}} \rightarrow L_{n}=L_{p} \cdot \sqrt{\frac{\mu_{n}}{\mu_{p}}}
$$

By subsituting Equation 5.60 into Equation 5.59:

$$
W_{n} \cdot L_{n}=W_{p} \cdot L_{p} \rightarrow \frac{W_{p}}{W_{n}}=\frac{L_{n}}{L_{p}}=\sqrt{\frac{\mu_{n}}{\mu_{p}}}
$$

Thus we can match both " $\mathrm{g}_{\mathrm{m}}$ " and "impedance" of PMOS and NMOS switching devices with the following steps:

1. Choose " $\mathrm{L}_{\mathrm{p}}$ " to minimum gate length specified by the chosen technology to ensure fastest PMOS operation

2. Scale NMOS device length " $\mathrm{L}_{\mathrm{n}}$ " to the value given by Equation 5.60 
3. Scale the PMOS to NMOS gate width ratio " $\mathrm{W}_{\mathrm{p}} / \mathrm{W}_{\mathrm{n}}$ " to the value given by Equation 5.61

\subsubsection{Impact on phase noise: tail current bias device}

Hajimiri revealed in his papers that the bias current tail transistor is a cause for phase noise degradation [10], [13]. Since the drain node of the bias current tail transistor is pulled up whenever each one of the two NMOS cross-coupled latch devices conducts, which corresponds to half the oscillation period; the drain node thus moves at twice the oscillation frequency.

Due to this frequency-doubling nature, noise close to even harmonics (instead of odd harmonics) of the oscillation frequency will be up/down-converted as phase noise at the output spectrum. This is accomplished by the oscillator ISF even order co-efficients; namely "c $c_{0}$ ", "c $c_{2}$ ", "c $c_{4}$ ", and so on. The effect of such phase noise degradation is illustrated as follows (see figure 5.10): 


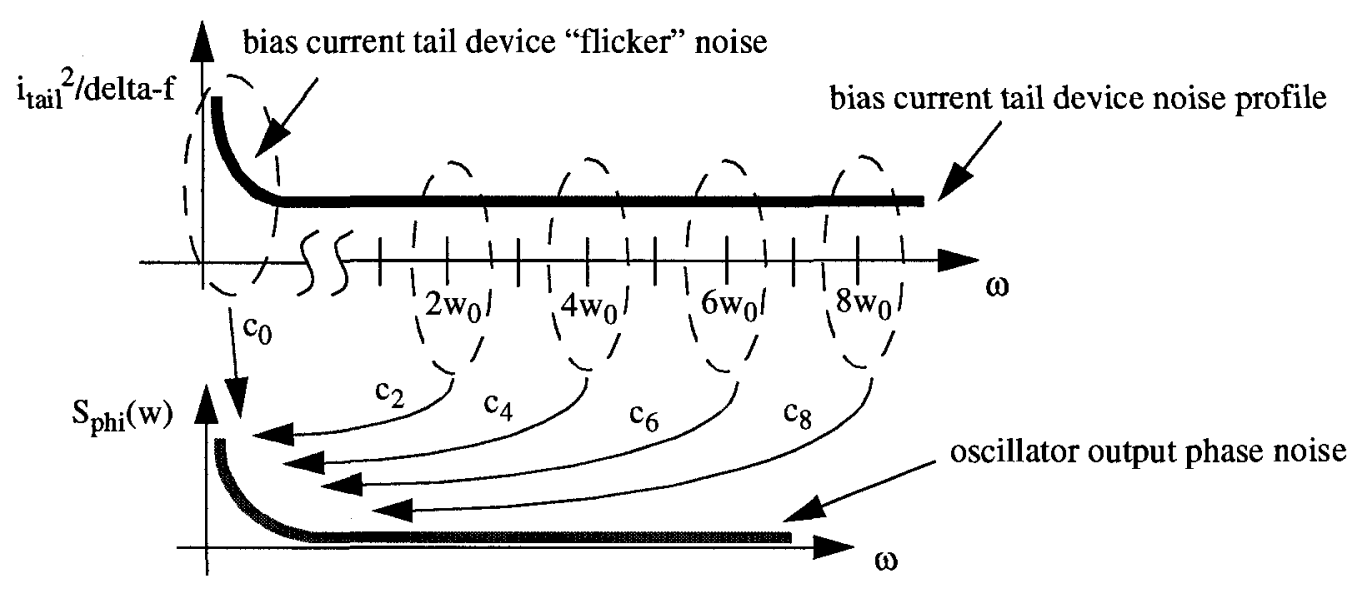

Figure 5.10: Up-conversion of flicker noise and down-conversion of noises in even order harmonics vicinity onto oscillator phase noise

In order to resolve this phase noise degradation issue, Hajimiri placed a shunt capacitor to the NMOS bias tail current device. The shunt capacitor acts as a low pass filter (i.e. if designed with a $-3 \mathrm{~dB}$ corner frequency much lower than the 2 nd harmonics of the oscillation frequency), and attenuates high frequency signals at the joined source node of the NMOS cross-coupled latch devices. Thus variation on the drain-source voltage of the NMOS bias tail current device is reduced, and channel-length modulation on the drain current is minimized (see Figure 5.11). 


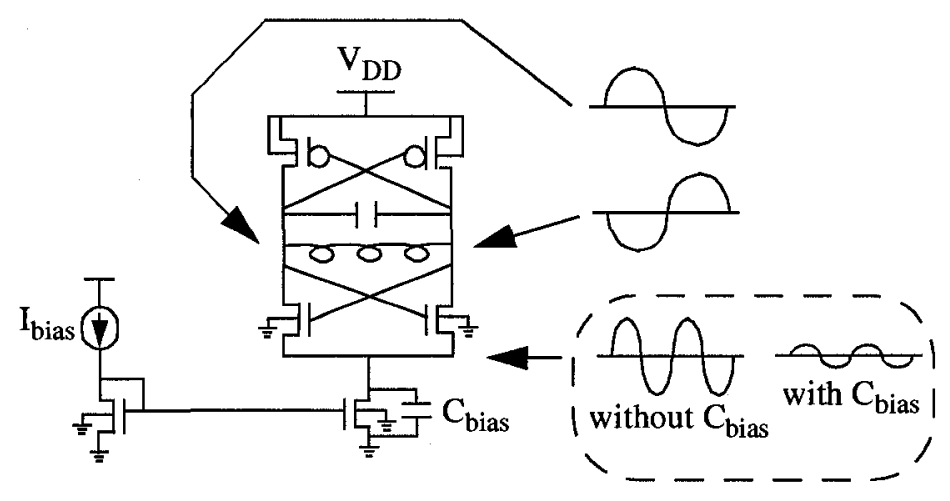

Figure 5.11: Complementary LC-VCO node voltages and effect of shunt capacitor " $\mathrm{C}_{\mathrm{bias}}$ " on bias NMOS Vds ripple reduction

In addition to the voltage variation reduction on the drain node of the NMOS bias tail current device, the placement of the shunt capacitor " $\mathrm{C}_{\text {bias }}$ " also reduced the duty cycle of both PMOS and NMOS cross-coupled latch devices (only NMOS devices are shown in Figure 5.12). In other words, less drain currents and corresponding drain current noises will be flowing through the oscillator circuit within each oscillation period. Thus phase noise of the LC-VCO circuit will be improved. 


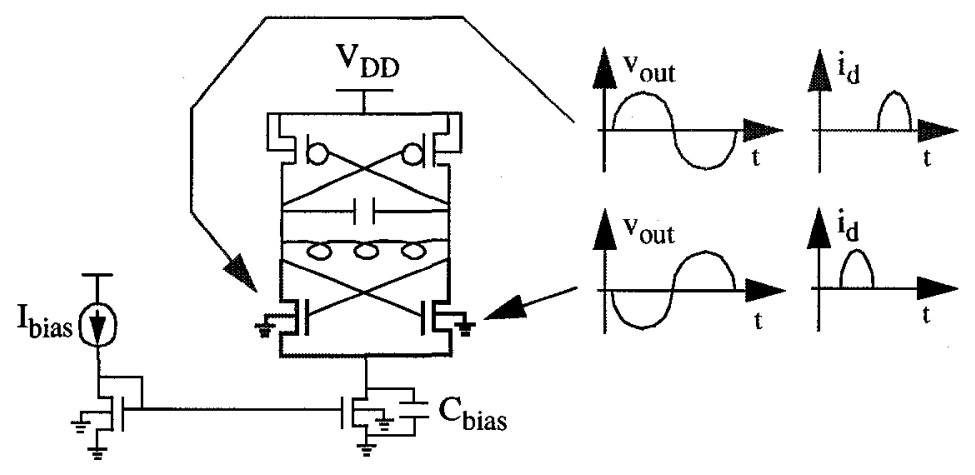

Figure 5.12: Cross-coupled NMOS device duty cycle reduction effect from shunt capacitor "C $\mathrm{C}_{\text {bias }}$ "

One disadvantage of this shunt capacitor implementation is the reduction on the oscillator output impedance at high frequency (i.e. impedance of a capacitor decreases as frequency increases). Thus it makes the oscillator circuit more susceptable to noise on the power supply " $\mathrm{V}_{\mathrm{DD}}$ ".

Finally, the shunt capacitor implementation only resolves the issue related to down-conversion of noise close to even harmonics of the circuit's oscillation frequency; but not the issue related to up-conversion of low frequency noise sources (i.e. flicker noise of NMOS bias tail current device). The solution for this issue can be addressed by adding some series resistance and extra shunt capacitance between the gates of the NMOS bias current mirror devices. Thus, this low pass filter will reduce the device noise contribution 
(i.e. beyond the LPF $3 \mathrm{~dB}$-corner) at a rate of $-20 \mathrm{~dB} / \mathrm{dec}$ de. But in order to filter out noise at very low frequencies, this shunt capacitor will most likely be implemented as an offchip component.

In conclusion, the relationship between NMOS current bias transistor and up/ down-conversions of flicker/white noise is applicable to both active and passive LC-VCO circuits. Since noise power is multiplied by the square of the current mirror ratio (see Chapter 2), extreme care must be taken on the choice of the bias current mirror ratio. This is a trade-off between the current required for oscillation speed, voltage headroom for $\mathrm{Vds}$ of NMOS bias current tail device, and phase noise performance caused by the low-frequency noise source up-conversion mechanism.

\subsection{Results Summary}

Oscillation frequency measurements on both active and passive LC-VCO circuits are close to their corresponding simulation results. The passive LC-VCO circuit has a frequency span of $+/-7.9 \%$ from the centre frequency of $4.9675 \mathrm{GHz}$. The active LC-VCO circuit has a frequency of $+/-22.16 \%$ from the centre frequency of $4.2295 \mathrm{GHz}$. 
Phase noise measurements on both active and passive LC-VCO circuits are much worse than their corresponding simulation results. The passive LC-VCO circuit has the best phase noise measurement of $-66.72 \mathrm{dBc} / \mathrm{Hz}$ and $-74.37 \mathrm{dBc} / \mathrm{Hz}$ at $100 \mathrm{kHz}$ and $1 \mathrm{MHz}$ away from the oscillation frequency of $5.24 \mathrm{GHz}$. The active LC-VCO circuit has the best phase noise measurement of $-63.62 \mathrm{dBc} / \mathrm{Hz}$ and $-66.8 \mathrm{dBc} / \mathrm{Hz}$ at $100 \mathrm{kHz}$ and $1 \mathrm{MHz}$ away from the oscillation frequency of $4.515 \mathrm{GHz}$.

Potential root causes of sub-optimal phase noise results and slight mismatches in oscillation frequencies and KVCO values include: single-ended phase noise measurement from differential buffer output nodes, incomplete parasitic modeling for simulations, lack of on-chip and off-chip decoupling capacitors for input/power/ground nodes, and inaccurate modeling for frequency tuning components of both active and passive LC-VCO circuits; namely the voltage-to-current converter circuit and the lateral PNP bipolar transistors.

A few solutions for phase noise performance improvement are included at the end of this chapter, which can be further investigated as future work. 


\section{CHAPTER 6 Conclusions}

\subsection{Summary Of Key Findings}

This thesis has explored the concept of active inductance generation through various gyrator-capacitor feedback circuit topologies. The concept has been verified with the construction of an "active-inductor"-capacitor voltage controlled oscillator circuit; whose architecture resembles a 2-stage differential ring oscillator circuit. For comparison purpose, a resonator based voltage controlled oscillator circuit is built with on-chip differential spiral inductor and PNP varactor devices; and is referred as a LC-VCO circuit. Both ring oscillator and LC-VCO circuits have been fabricated with TSMC180nm technology. 
From measurements, the differential ring oscillator circuit has a frequency span of $+/-22.16 \%$ from the centre frequency of $4.2295 \mathrm{GHz}$; and best phase noise measurements of $-63.62 \mathrm{dBc} / \mathrm{Hz}$ and $-66.8 \mathrm{dBc} / \mathrm{Hz}$ at $100 \mathrm{kHz}$ and $1 \mathrm{MHz}$ away from the oscillation frequency of $4.515 \mathrm{GHz}$. These measured results are comparable to their corresponding postlayout simulation results, with the exception of phase noise measurement probed at $1 \mathrm{MHz}$ away from various oscillation frequency settings. The largest discrepancies between simulated and measured results is about $21.75 \mathrm{~dB}$.

From measurements, the LC-VCO circuit has a frequency span of $+/-7.9 \%$ from the centre frequency of $4.9675 \mathrm{GHz}$; and best phase noise measurements of $-66.72 \mathrm{dBc} / \mathrm{Hz}$ and $-74.37 \mathrm{dBc} / \mathrm{Hz}$ at $100 \mathrm{kHz}$ and $1 \mathrm{MHz}$ away from the oscillation frequency of $5.24 \mathrm{GHz}$. These measured results are comparable to their corresponding post-layout simulation results, with the exception of phase noise measurements made at both $100 \mathrm{kHz}$ and $1 \mathrm{MHz}$ away from various oscillation frequency settings. The largest discrepancies between simulated and measured phase noise results (at $1 \mathrm{MHz}$ offset) is about $40.47 \mathrm{~dB}$.

Potential root causes of discrepancies between simulated and measured results include: single-ended phase noise measurement from differential buffer output nodes, incomplete parasitic modeling for simulations, lack of on-chip decoupling capacitors for 
on-chip power and ground buses, and inaccurate modeling for frequency tuning components of both active and passive LC-VCO circuits; namely the voltage-to-current converter circuit and the PNP varactor devices.

However, such potential root causes for sub-optimal phase noise measurements will need to be validated with future research.

\subsection{Future Work}

Potential future investigations in the area of active inductance generation and onchip oscilator circuit design includes:

1. Validate potential root cause of sub-optimal phase noise performance with solutions proposed in Chapter 5

2. Improve on phase noise performance on both diffrential ring oscillator and LC-VCO circuits

3. Explore the application of differential ring oscillator to phase lock loop circuits 


\section{References}

[1] D. B. Leeson, "A Simple Model of Feedback Oscillator Noise Spectrum", Proceedings Of The IEEE, VOL. 54, pp. 329-330, February 1966

[2] T.N. Rao, P. Gary, R.W. Newcomb, "Equivalent Inductance and Q of a Capacitor-Loaded Gyrator”, IEEE Journal of Solid-State Circuits, VOL. 2, No. 3, pp. 32-33, March 1967

[3] A. Rofougaran, J.J. Rael, M. Rofougaran, A. Abidi, "A $900 \mathrm{MHz}$ LC-Oscillator with Quadrature Outputs in lum CMOS", Proceedings of ISSCC, pp. 392-393, San Francisco, CA, 1996

[4] Behzad Razavi, "A Study of Phase Noise in CMOS Oscillators", IEEE Journal of SolidState Circuits, VOL. 31, NO. 3, pp. 331-343, March 1996.

[5] A. Hajimiri, T. H. Lee, "A General Theory of Phase Noise in Electrical Oscillators", IEEE Journal of Solid-State Circuits, VOL. 33, NO. 2, pp. 179-194, February 1998

[6] A. Hajimiri, T. H. Lee, "Corrections to "A General Theory of Phase Noise in Electrical Oscillators", IEEE Journal of Solid-State Circuits, VOL. 33, NO. 6, June 1998

[7] J.E. Post, Jr., I.R. Linscott, M.H. Oslick, "Waveform symmetry properties and phase noise in oscillators", IEE Electronics Letters, VOL. 34, NO. 16, August 1998

[8] Behzad Razavi, RF Microelectronics, Prentice Hall Inc., 1998

[9] Ali M. Niknejad, Robert G. Meyer, "Analysis, Design, and Optimization of Spiral Inductors and Transformers for Si RF IC's", IEEE Journal of Solid-State Circuits, VOL. 33, NO. 10, October 1998

[10] A. Hajimiri, T. H. Lee, "Design Issues in CMOS Differential LC Oscillators", IEEE Journal of Solid-State Circuits, VOL. 34, NO. 5, May 1999

[11] A. Hajimiri, S. Limotyrakis, T.H. Lee, "Jitter and Phase Noise in Ring Oscillators", IEEE Journal of Solid-State Circuits, VOL. 34, NO. 6, June 1999

[12] Ali M. Niknejad, Robert G. Meyer, Inductors and Transformers for Si RF ICs, Kluwar Academic Publishers, 2000

[13] A. Hajimiri, T.H. Lee, "Oscillator Phase Noise: A Tutorial”, IEEE Journal of Solid-State Circuits, VOL. 35, NO. 3, March 2000

[14] HongMo Wang, "A Solution for Minimizing Phase Noise in Low-Power Resonator-Based Oscillators”, IEEE International Symposium on Circuits and Systems, May 28-31, 2000 
[15] J.J. Rael, Asad A. Abidi, "Physical Processes of Phase Noise in Differential LC Oscillators", IEEE Custom Integrated Circuits Conference, 2000

[16] Pietro Andreani, Sven Mattisson, "On the Use of MOS Varactors in RF VCO's", IEEE Journal of Solid-State Circuits, VOL. 35, NO. 6, June 2000

[17] Qiuting Huang, "Phase Noise to Carrier Ratio in LC Oscillators", IEEE Transactions on Circuits and Systems I: Fundamental Theory and Applications, VOL. 47, NO. 7, July 2000

[18] Paul R. Gray, Paul J. Hurst, Stephen H. Lewis, Robert G. Meyer, Analysis And Design Of Analog Integrated Circuits, John Wiley \& Sons Inc., 2001

[19] D. Ham, A. Hajimiri, "Concepts and Methods in Optimization of Integrated LC VCOs", IEEE Journal of Solid-State Circuits, VOL. 36, NO. 6, June 2001

[20] Emad Hegazi, Henrik Sjoland, Asad A. Abidi, "A Filtering Technique to Lower LC Oscillator Phase Noise”, IEEE Journal of Solid-State Circuits, VOL. 36, NO. 12, December 2001

[21] Salvatore Levantino, Carlo Samori, Alfio Zanchi, Andrea L. Lacaita, "AM-to-PM Conversion in Varactor-Tuned Oscillators", IEEE Transactions on Circuits and Systems II: Analog and Digital Signal Processing, VOL. 49, NO. 7, July 2002

[22] John Rogers, Calvin Plett, Radio Frequency Integrated Circuit Design, Artech House, 2003

[23] Emad Hegazi, "Varactor Characteristics, Oscillator Tuning Curves, and AM-FM Conversion”, IEEE Journal of Solid-State Circuits, VOL. 38, NO. 6, June 2003

[24] Thomas H. Lee, The Design of CMOS Radio-Frequency Integrated Circuits, 2nd edition, Cambridge University Press, 2004

[25] Adel S. Sedra, Kenneth C. Smith, Microelectronic Circuits, 5th edition, Oxford University Press, 2004

[26] Albert Jerng, Charles G. Sodini, "The Impact of Device Type and Sizing on Phase Noise Mechanisms", IEEE Journal of Solid-State Circuits, VOL. 40, NO. 2, February 2005

[27] Babak Soltanian, Peter R. Kinget, "Tail Current-Shaping to Improve Phase Noise in LC Voltage-Controlled Oscillators", IEEE Journal of Solid-State Circuits, VOL. 41, NO. 8, August 2006

[28] Asad A. Abidi, "Phase Noise and Jitter in CMOS Ring Oscillators", IEEE Journal of Solid-State Circuits, VOL. 41, NO. 8, August 2006

[29] John Rogers, Calvin Plett, Foster Dai, Integrated Circuit Design For High-Speed Frequency Synthesis, Artech House Inc., 2006 